Florida International University FIU Digital Commons

7-2-2015

\title{
Macular Pigment and Lens Optical Density Measurements-Evaluating a Flicker Machine with Novel Features
}

Anirbaan Mukherjee

Florida International University, anirbaan8280@gmail.com

DOI: $10.25148 /$ etd.FIDC000113

Follow this and additional works at: https://digitalcommons.fiu.edu/etd

Part of the Physics Commons

\section{Recommended Citation}

Mukherjee, Anirbaan, "Macular Pigment and Lens Optical Density Measurements-Evaluating a Flicker Machine with Novel Features" (2015). FIU Electronic Theses and Dissertations. 2187.

https://digitalcommons.fiu.edu/etd/2187

This work is brought to you for free and open access by the University Graduate School at FIU Digital Commons. It has been accepted for inclusion in FIU Electronic Theses and Dissertations by an authorized administrator of FIU Digital Commons. For more information, please contact dcc@fiu.edu. 


\title{
FLORIDA INTERNATIONAL UNIVERSITY
}

\author{
Miami, Florida
}

\section{MACULAR PIGMENT AND LENS OPTICAL DENSITY MEASUREMENTS- EVALUATING A FLICKER MACHINE WITH NOVEL FEATURES}

\author{
A dissertation submitted in partial fulfillment of the \\ requirements for the degree of \\ DOCTOR OF PHILOSOPHY \\ in \\ PHYSICS \\ by
}

Anirbaan Mukherjee 
To: Dean Michael R. Haithaus

College of Arts and Sciences

This dissertation, written by Anirbaan Mukherjee, and entitled Macular Pigment and Lens Optical Density Measurements-Evaluating a Flicker Machine with Novel Features, having been approved in respect to style and intellectual content, is referred to you for judgment.

We have read this dissertation and recommend that it be approved.

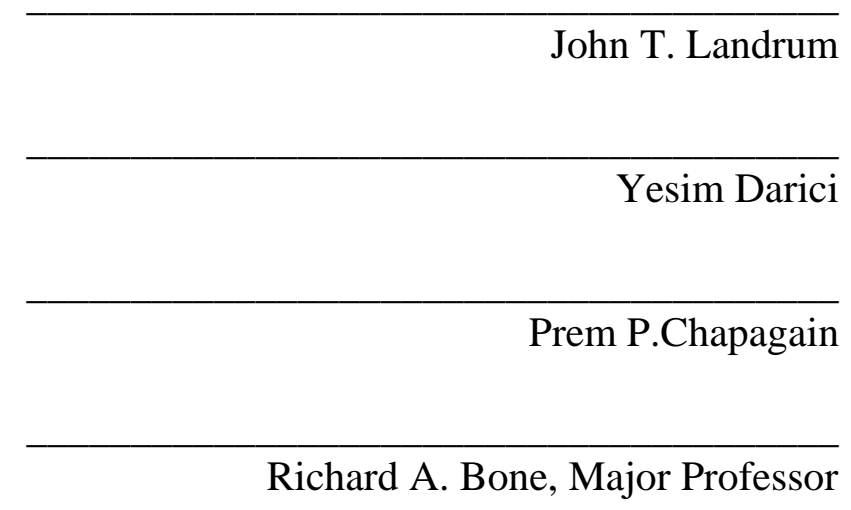

Date of Defense: July 02, 2015

The dissertation of Anirbaan Mukherjee is approved.

$\begin{array}{r}\hline \begin{array}{r}\text { Dean Michael R. Haithaus } \\ \text { College of Arts and Sciences }\end{array} \\ \hline \begin{array}{r}\text { Dean Lakshmi N. Reddi } \\ \text { University Graduate School }\end{array}\end{array}$

Florida International University, 2015 


\section{DEDICATION}

I dedicate my dissertation to my advisor Dr. Richard A. Bone, my grandfather Late Ajit Ratan Majumder, my father Mr. Pronab Kumar Mukherjee, my mother Mrs. Rupasree Mukherjee and my sister Miss Oindree Priyadarshini Mukherjee. These five individuals have had profound influence on my life and have been instrumental in carving the personality which defines me today, ready to face life at large. 


\section{ACKNOWLEDGMENT}

I would like to express my sincerest acknowledgement to my advisor and mentor Dr. Richard A. Bone. I am indebted to Dr. Bone for allowing me to pursue my $\mathrm{PhD}$ research under his guidance. The various conferences that I have attended domestically and internationally presenting my research done under the aegis of Dr. Bone has helped me interact and establish network with top level researchers and scientist in the area of vision science.

I also acknowledge the time and support that I have been fortunate to avail of each of my committee members Dr. John T. Landrum, Dr. Yesim Darici and Dr. Prem P. Chapagain.

I would like to thank Mr. Michael Favish of Guardion Health Sciences and Beneseed Co. Ltd. for providing support for the research conducted towards my PhD work.

I deeply appreciate Late Mr. Carlos Orta for his flexibility of accommodating my requests for design fabrications in the midst of his busy schedule and Mr. Wenlue Huang the electronics engineer for being readily available when approached with urgency.

I would like to thank the Department of Physics at Florida International University for supporting my entire $\mathrm{PhD}$ work.

Last but not the least would like to thank all the subjects that have participated in the study. 


\begin{abstract}
OF THE DISSERTATION
MACULAR PIGMENT AND LENS OPTICAL DENSITY MEASUREMENTSEVALUATING A FLICKER MACHINE WITH NOVEL FEATURES
\end{abstract}

by

Anirbaan Mukherjee

Florida International University, 2015

Miami, Florida

Professor Richard A. Bone, Major Professor

Age related macular degeneration (AMD) is one of the leading causes of blindness amongst the elderly. Macular pigment (MP) in the retina has been established to protect individuals against AMD. Improving levels of MP by diet or supplements is the constant quest of clinical practitioners and researchers, thus necessitating development of instruments capable of repeatable and reliable MP measurement. Cataract, a consequence of the rising opacity levels of the lens with age is one of the other major causes of blindness in the world. Mapcatsf, a LEDbased microprocessor-controlled heterochromatic flicker photometer (HFP) using photopic vision is capable of measuring the levels of MP and the opacity of the lens in terms of optical density.

Test-retest measurements conducted on 83 subjects were analyzed for repeatability in macular pigment optical density (MPOD) measurements. 
Reliability of the lens optical density (LOD) measurements were tested and compared with those obtained at absolute scotopic thresholds for 25 individuals. A supplement study with 32 individuals both in the young $(<30)$ and old $(>50)$ age groups for 6 months further established Mapcatsf's capacity to monitor changing levels of MP in individuals. As an overall outcome, high levels of repeatability and reliability were obtained in MPOD and LOD measurements establishing Mapcatsf as an instrument for use in clinical settings in the future. 


\section{CONTENTS}

CHAPTER

PAGE

1. Chapter 1.

Macular Pigment and Eye Health...........................................................1

Section 1.1 What is the macular pigment?............................................. 1

Section 1.2 Absorbance spectrum of the macular pigment.........................3

Section 1.3 Macular pigment in relation to proper eye health....................4

Section 1.4 Methods and techniques for measuring the macular pigment..7

2. Chapter 2.

Instrument Design and Subject Testing Procedure.................................12

Section 2.1 Instrument Design.........................................................12

Section 2.2.1 Theory for MPOD measurement......................................18

Section 2.2.2 Theory for LOD or LEA measurement...............................23

Section 2.2.3 LEA computation based on templates from excised lens.....24

Section 2.3 Subject Testing procedure..................................................26

3. Chapter 3.

Pathway to Establishing Credibility of Mapcatsf......................................32

Section 3.1 Test Retest Results.............................................................32

Section 3.2 Testing of Mapcatsf beyond

test-retest to determine its reliability..................................36

4. $\quad$ Chapter 4.

Effects of Shifts in Spectrum of the Component LEDs. 
Section 4.1 Introduction..............................................................4

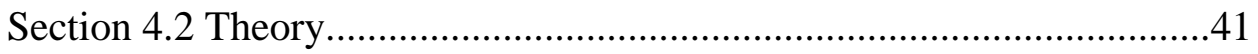

Section 4.3 Simulations.................................................................43

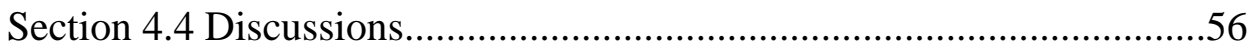

5. $\quad$ Chapter 5.

Variation of Peak Macular Pigment Optical Density with

Age measured using Heterochromatic Flicker Photometry......................59

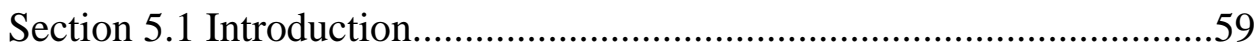

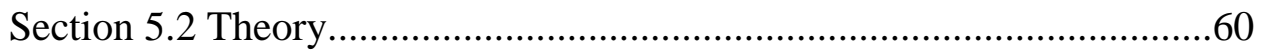

Section 5.3 Experiment................................................................63

Section 5.4 Discussion and Conclusion..................................................66

6. Chapter 6 .

Validity of Mapcatsf's capacity to measure lens optical density...............69

Section 6.1 Importance of developing reliable lens density measuring techniques....................................................69

Section 6.2 Scotopic Measurements: Theory.......................................72

Section 6.3 Scotopic Measurements: Instrument design.......................75

Section 6.4.1 Design and Plan of the Study..........................................77

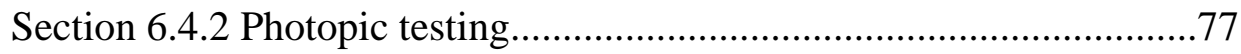

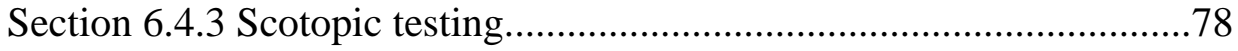

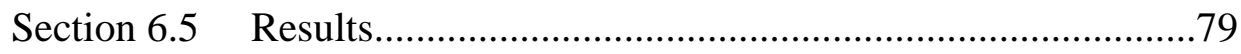

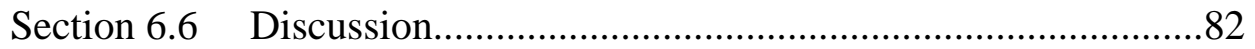


7. Chapter 7.

Credibility of Mapcatsf for monitoring MPOD levels, of an individual consuming lutein or its derivative supplement

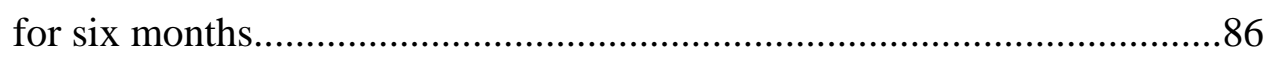

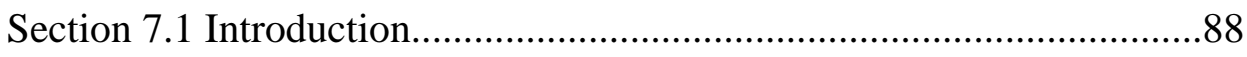

Section 7.2 Overview of the Supplements..........................................89

Section 7.3 Subject recruitment, profile and elimination criteria..............89

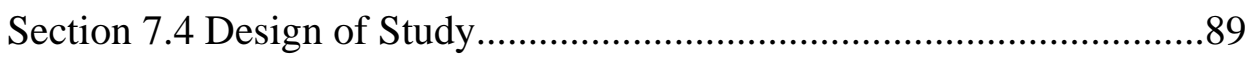

Section 7.5 Testing Procedure...............................................................90

Section 7.6 MPOD levels at 8 weeks interval.......................................90

Section 7.7 Response to the supplementation......................................95

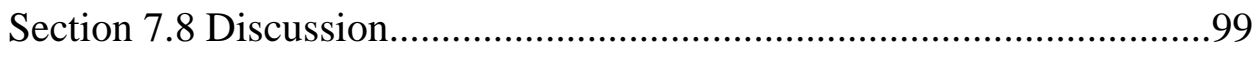

8. Chapter 8

Protective Role of Lutein found on the Lens in the Human Eye.............105

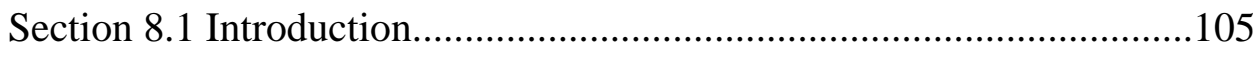

Section 8.2 Methods: subject recruitment..........................................107

Section 8.3 MPOD and LOD measurement.......................................107

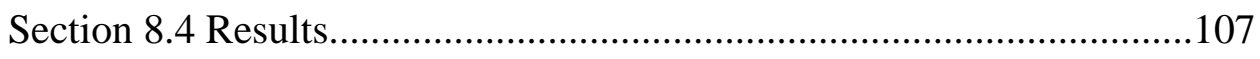

Section 8.5 Discussion............................................................... .112 
9. Chapter 9

Role of Involuntary eye movements in designing an optimum

gaze facilitating stimulus............................................................ 115

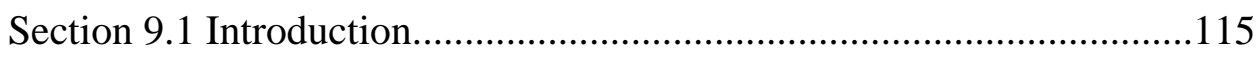

Section 9.2 Experimental Setup.......................................................116

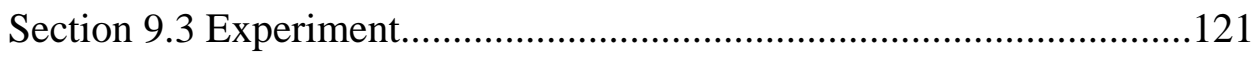

Section 9.4 Data Analysis.................................................................123

Section 9.5 Discussion and Conclusion..............................................127

10. Chapter 10

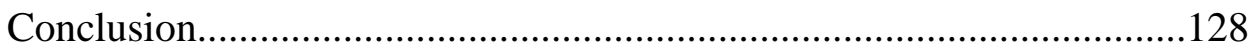

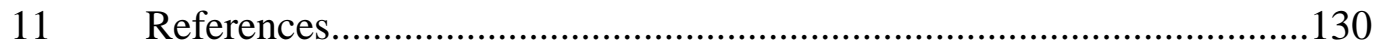

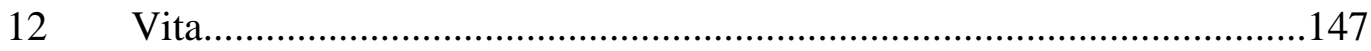




\section{LIST OF TABLES}

TABLE

PAGE

6.1 LEAs measured under the two separate viewing conditions are presented. The columns 5 and 6 consists the recalculated LEAs at extreme cone weights of 0.47 and 16.5 respectively.

7.6.1 The baseline and measured MPODs at 8 weeks interval of male subjects between 18 and 30 years during the supplementation period. The subjects on Lutein are titled as $\mathrm{L}$ and the one provided the Micromic are titled $\mathrm{M}$.

7.6.2 The baseline and measured MPODs at 8 weeks intervals of female subjects between 18 and 30 years during the supplementation period. The subjects on Lutein are titled as $\mathrm{L}$ and the ones provided the Micromic are titled $\mathrm{M}$.

7.6.4 The baseline and measured MPODs at 8 weeks interval of female subjects 50 years or above during the supplementation period. The subjects on Lutein are titled as $\mathrm{L}$ and the ones provided the Micromic are titled $\mathrm{M}$.

7.7.1 Response of male subjects in the younger age group to the supplement consumption considering the baseline and final MPOD values from table 7.6.1. The rate of change involves all the interim measurements done during the 6 month study.

7.7.2 Response of female subjects in the younger age group to the supplement consumption considering the baseline and final MPOD values from table 7.6.2. The rate of change involves all the interim measurements done during the 6 month study. 
7.7.3 Response of the male subjects in the older age group to the supplement consumption considering the baseline and the final MPOD values from table 7.6.3. The rate of change involves all the interim measurements done during the 6 month study.

7.7.4 Response of the female subjects in the older age group to the supplement consumption considering the baseline and the final MPOD values from table 7.6.4. The rate of change involves all the interim measurements done during the 6 month study.

7.7.5 Overall response to the supplementation across the age groups.

7.8.1 Subjects on the Lutein supplement with significant change in their MPOD levels at the end of the study.

7.8.2 Subjects on the Micro supplement with significant change in their MPOD levels at the end of the study.

7.8.3 List of subjects whose MPOD levels was not changed significantly at the end of the study.

7.8.4 Subjects on Lutein supplement who had shown an overall significant change and a significant rate of change in their MPODs

7.8.5 Subjects on the Micro supplement who had shown an overall significant change and a significant rate of change in their MPODs 
8.1 Age- and gender-based classification of the participating subjects.

8.2 Average of MPOD obtained from test retest for the subjects 108

8.3 Average of LOD obtained from test retest for the subjects. 109

9.1 Variation in gaze for the three types of stimuli tested. 


\section{LIST OF FIGURES}

FIGURE PAGE

1.1 The location of the macular pigment in the macaque retina. Image courtesy: Fig1 (a) of Malinow et al (1980)

1.2 The spatial distribution of the total carotenoid or the macular pigment in the retina is shown by the purple line.The cyan line shows the ratio of lutein to zeaxanthin.In the central retina zeaxanthin is the dominant carotenoid and the ratio of mesozeaxanthin to zeaxanthin is shown by the green line. Image courtesy Bone et al 1988

1.3 The normalized optical density spectrum of the macular pigment, obtained from liposome-bound zeaxanthin and lutein as published in Bone et al in 1992

1.4 (a) Sample field as seen by a person with no vision anomaly

2.1 The luminous efficiency functions plotted for centrally viewed fields of 10 degree (dashed line) and 2 degree (solid line) in diameter

2.2 The optical setup of Mapcatsf.The interior of the sphere $\mathrm{S}_{1}$ is illuminated by the blue and green LEDs. The intensity settings of the blue and green LEDs at flicker null are measured by the photodioide PD. The apertures on disk D provide the $1.5^{\circ}$ and $15^{\circ}$ stimuli as viewed by the telescopic setup which consists of the lenses $\mathrm{L}_{1}$ acting as the objective and $\mathrm{L}_{2}$ acting as the eyepiece along with the field stop $\mathrm{F}$ and aperture A.The front surface of $\mathrm{D}$ is illuminated by the LEDs on the sphere $\mathrm{S}_{2}$. Image courtesy from SPIE,JBO 
2.3 The ray diagram explaint he fact that when the distance between the nodal point of the eye and the eyepiece lens $L_{2}$ is equal to the focal length of $L_{2}$, the angle subtended by the stimulus imaged on the retina is the same regardless of the position of the second image I2, which is adjusted based on the subject's accommodation.

2.4 The plots of $P$ vs $\log \left(\frac{\phi_{B F}}{\phi_{B P}}\right)$ for LEAs at 5 years intervals between 20 and 90 years.

2.5 The polynomial fit used to obtained the lens equivalent age or LEA from the intensity ratios of the blue and the green LED sources detected bv the photodetector at perifoveal flicker null settings, $\frac{\phi_{B P}}{\phi_{G}}$

2.6 The plots of $\frac{\phi_{B P}}{\phi_{G}}$ vith LEA, using the three different

templates. The solid green line is obtained by using the template suggested by Sagawa and Takahashi. The red and blue dashed lines are obtained as a result of using template suggested by Pokorny and van de Kraats respectively

2.7 The appearance of the $15^{\circ}$ stimulus to the subject when the flicker null/minimum condition is reported in the periphery. 
3.1 Graph showing the Bland Altman analysis for the 83 subjects where the difference in MPODs measured during the test and retest is plotted with respect to the mean of the MPODs measured. The solid line depicts the mean of the test-retest and the dashed horizontal line are the limits of agreement

3.2 The scatter plot along with the regression analysis for MPODs measured during retest with respect to the MPODs measure during test for 83 subjects

3.3 The LEA or lens equivalent age is plotted as a function of the subject's actual age.The solid line is a line of slope unity. The points above the line are the ones for the subjects whose LEAs were more than their actual age and the points that are below are the ones for whom the LEAs were less than their actual age

4.1 a Variation in the percentage difference of MPOD observed for an individual with an LEA of 20 years when the blue LED is shifted by $5 \mathrm{~nm}$. The green lineis for the variation observed when the blue LED is moved by $+5 \mathrm{~nm}$ and the red line is for the variation observed when the blue LED is moved by $-5 \mathrm{~nm}$

$4.1 \mathrm{~b}$ Variation in the percentage difference of MPOD observed for an individual with an LEA of 40 years when the blue LED is shifted by $5 \mathrm{~nm}$. The green line is for the variation observed when the blue LED is moved by $+5 \mathrm{~nm}$ and the red line is for the variation observed when the blue LED is moved by $-5 \mathrm{~nm}$ 
4.1 c Variation in the percentage difference of MPOD observed for an individual with an LEA of 60 years when the blue LED is shifted by $5 \mathrm{~nm}$. The green line is for the variation observed when the blue LED is moved by $+5 \mathrm{~nm}$ and the red line is for the variation observed when the blue LED is moved by $-5 \mathrm{~nm}$.

$4.1 \mathrm{~d}$ Variation in the percentage difference of MPOD observed for an individual with an LEA of 80 years when the blue LED is shifted by $5 \mathrm{~nm}$. The green line is for the variation observed when the blue LED is moved by $+5 \mathrm{~nm}$ and the red line is for the variation observed when the blue LED is moved by $-5 \mathrm{~nm}$

4.2 a Variation in the percentage difference of MPOD observed for an individual with an LEA of 20 years when the green LED is shifted by $5 \mathrm{~nm}$. The green line is for the variation observed when the green LED is moved by $+5 \mathrm{~nm}$ and the red line is for the variation observed when the green LED is moved by $-5 \mathrm{~nm}$

$4.2 \mathrm{~b}$ Variation in the percentage difference of MPOD observed for an individual with an LEA of 40 years when the green LED is shifted by $5 \mathrm{~nm}$. The green line is for the variation observed when the green LED is moved by $+5 \mathrm{~nm}$ and the red line is for the variation observed when the green LED is moved by $-5 \mathrm{~nm}$

4.2 c Variation in the percentage difference of MPOD observed for an individual with an LEA of 60 years when the green LED is shifted by $5 \mathrm{~nm}$. The green line is for the variation observed when the green LED is moved by $+5 \mathrm{~nm}$ and the red line is forthe variation observed when the green LED is moved by $-5 n m$ 
FIGURE

PAGE

$4.2 \mathrm{~d}$ Variation in the percentage difference of MPOD observed for an individual with an LEA of 80 years when the green LED is shifted by $5 \mathrm{~nm}$, The green line is for the variation observed when the green LED is moved by $+5 \mathrm{~nm}$ and the red line is for the variation observed when the green LED is moved by $-5 \mathrm{~nm}$

4.3 The percentage differences in LEA due to shift in the green LED by $\pm 5 \mathrm{~nm}$. The green line is for the percentage differences in the LEAs that will be the result when the peak of the green LED is moved by+5nm.Similarly the red line is for the percentage difference in the LEA that will result when the green LED is moved by-5nm

4.4 The percentage differences in LEA due to shift in the blue LED by $\pm 5 \mathrm{~nm}$. The green line is for the percentage differences in the LEAs that results when the peak of the blue LED is moved by+5nm. Similarly the red line is for the percentage difference in the LEA that will result when the blue LEDis moved by $-5 \mathrm{~nm}$

4.5 a Variation in the percentage difference of MPOD observed for an individual with an LEA of 20 years when the blue LED is shifted by $1 \mathrm{~nm}$. The green line is for the variation observed when the blue LED is moved by $+1 \mathrm{~nm}$ and the red line is for the variation observed when the blue LED is moved by $-1 \mathrm{~nm}$

$4.5 \mathrm{~b}$ Variation in the percentage difference of MPOD observed for an individual with an LEA of 40 years when the blue LED is shifted by $1 \mathrm{~nm}$. The green line is for the variation observed when the blue LED is moved by $+1 \mathrm{~nm}$ and the red line is for the variation observed when the blue LED is moved by $-1 \mathrm{~nm}$ 
FIGURE

PAGE

4.5 c Variation in the percentage difference of MPOD observed for an individual with an LEA of 60 years when the blue LED is shifted by $1 \mathrm{~nm}$. The green line is for the variation observed when the blue LED is moved by $+1 \mathrm{~nm}$ and the red line is for the variation observed when the blue LED is moved by $-1 \mathrm{~nm}$

$4.5 \mathrm{~d}$ Variation in the percentage difference of MPOD observed for an individual with an LEA of 80 years when the blue LED is shifted by $1 \mathrm{~nm}$. The green line is for the variation observed when the blue LED is moved by +1 $\mathrm{nm}$ and the red line is for the variation observed when the blue LED is moved by $-1 \mathrm{~nm}$

4.6 a Variation in the percentage difference of MPOD observed for an individual with an LEA of 20 years when the green LED is shifted by $1 \mathrm{~nm}$. The green line is for the variation observed when the green LED is moved by $+1 \mathrm{~nm}$ and the red line is for the variation observed when the green LED is moved by $-1 \mathrm{~nm}$

$4.6 \mathrm{~b}$ Variation in the percentage difference of MPOD observed for an individual with an LEA of 40 years when the green LED is shifted by $1 \mathrm{~nm}$. The green line is for the variation observed when the green LED is moved by $+1 \mathrm{~nm}$ and the red line is for the variation observed when the green LED is moved by $-1 \mathrm{~nm}$

4.6 c Variation in the percentage difference of MPOD observed for an individual with an LEA of 60 years when the green LED is shifted by $1 \mathrm{~nm}$. The green line is forthe variation observed when the green LED is moved by $+1 \mathrm{~nm}$ and the red line is for the variation observed when the green LED is moved by $-1 \mathrm{~nm}$ 
FIGURE

4.6 d Variation in the percentage difference of MPOD observed for an individual with an LEA of 80 years when the green LED is shifted by $1 \mathrm{~nm}$. The green line is for the variation observed when the green LED is moved by $+1 \mathrm{~nm}$ and the red line is for the variation observed when the green LED is moved by $-1 \mathrm{~nm}$

4.7 Variation in percentage difference in LEA due to shift in the blue LED by $\pm 1 \mathrm{~nm}$.The green line is for the percentage differences in the LEAs that results when the peak of the blue LED is moved by $+1 \mathrm{~nm}$. Similarly the red line is for the percentage difference in the LEA that will result when the blue LED is moved by $-1 \mathrm{~nm}$

4.8 Variation in percentage difference in LEA due to shift in the green LED by $\pm 1 \mathrm{~nm}$. The green line is for the percentage differences in the LEAs that results when the peak of the green LED is moved by+1nm.Similarly the red line is for the percentage difference in the LEA that will result when the green LED is moved by $-1 \mathrm{~nm}$

5.1 The age corrected luminous efficiency function for a centrally viewed 10 degree stimulus.

5.2 Plot of peak MPOD $P$ with $\log \left(\frac{\phi_{B F}}{\phi_{B P}}\right)$ for 50,30 and 15 year olds.

5.3 The scatter plot of simulated peak MPOD values with the LEAs generated from random numbers.

5.4 Plot of $\log \left(\frac{\phi_{B F}}{\phi_{B P}}\right)$ values versus LEAs generated from random numbers 
5.5 Scatter Plot of MPOD with age generated from random Nos. The red dots or the partially corrected MPOD values are obtained when the values of $\log \left(\frac{\phi_{B F}}{\phi_{B P}}\right)$ are used for the polynomial fit with coefficients fixed for that of a 32 year old in eq. 1 in section 4.2. The red line gives the regression analysis of the variation of MPOD with age. The black dots depict the true simulated MPODs values of the subjects generated from the random numbers on Sigmaplot.

5.6 The plot of MPOD measured versus the LEAs measured while testing for repeatability of Mapcatsf. The regression line has Eq. of the $y=0.352+3.155 \times 10^{-3} x$.

6.1 The polynomial fit to the plot of LEA with F.

6.2 The central gaze fixating stimulus used for absolute scotopic threshold measurements.75

6.3 The layout and optical setup for the scotopic lens density measurement

6.4 A sample scatter plot obtained for a participating subject for one of the LEDs tested. The inverse sigmoidal curve of the form as given in Eq.(6) was fitted to obtain the absolute scotopic threshold at the $50 \%$ viewing criterion. 
FIGURE

PAGE

6.5 The scatter plot of photopic LEAs obtained from Mapcatsf versus thescotopic LEAs obtained at absolute threshold. The solid line is of slope unity. The dashed line is the regression line of Eq. $y=0.833 x+3.945$.

6.6 The Bland Altman plot used for testing for levels of agreement of the results between the LEAs obtained under the two separate viewing conditions. The mean difference which was 2.00 years is depicted by the solid horizontal line and the dashed lines are for depicting Mean \pm 2 SD.

8.1 Scatter plot of LOD with MPOD for all subjects. The regression line shown in red is given by the Eq.: $\mathrm{y}=0.528+0.267 \mathrm{x}$, with a $\mathrm{p}<0.0175$.

8.2 Regression lines for the scatter plots of LOD with MPOD for the three age groups. For the group of subjects between 18 and 30 years the Eq. of the line was $y=0.514+0.056 x$ (shown in red), $\mathrm{p}$ value $=0.3863$; For the group of subjects between 30 and 50 years the Eq. of the regression line was $y=0.469+0.341 \mathrm{x} \quad$ (shown in blue), $\mathrm{p}$ value $=0.0867$; and the Eq. of the regression line for the group of subjects between 50 and 80 years was $y=0.866+0.107 x$ (shown in green), $p$ value $=0.625$.

8.3 Regression lines for scatter plots of LOD with MPOD for females in the three age groups. For the age group of females between 18 and 30 years the regression line has an Eq.: $y=0.494+0.028 x$ (shown in red), $p$ value $=0.784$. For the age group of females between 30 and 50 years the regression line has an Eq. $y=0.775-0.228 x$ (shown in blue), p-value $=0.507$. For females in the age group of 50 to 80 years the regression line: $y=0.836+0.312 x$ (shown in green), $p$-value $=0.312$. 
8.4 Regression lines for scatter plots of LOD with MPOD for males in the three age groups. For the age group of males between 18 and 30 years the regression line has an Eq.: $y=0.551+0.009 x$ (shown in red), $p$-value $=0.895$. For the age group of males between 30 and 50 years the regression line has an Eq. $y=0.432$ $+0.423 x$ (shown in blue), $p$-value $=0.0914$. For males in the age group of 50 to 80 years the regression line: $\mathrm{y}=$ $0.885+0.019 x$ (shown in green), $p$ value $=0.962$.

9.1 The types of configurations of foveal stimuli tested for facilitating minimum fixational eye movements.

9. 2 The screen and projector setup

9.3 The hardware components of the eyetracker

9.6 A sample of Transformed gaze coordinates in the $\mathrm{Y}$ direction prior to analysis.

9.7 A sample of Transformed gaze coordinates in the $\mathrm{X}$ direction prior to analysis. 


\section{Chapter 1}

\section{Macular Pigment and Eye Health}

\section{Section 1.1}

\section{What is the macular pigment?}

Macular pigment(MP) is a non-bleaching yellow-color pigment that is found in the primate retina. ${ }^{1}$ The pigment is primarily concentrated in the foveal region of the retina ${ }^{2}$ as shown in Fig. 1.1 below. The fovea of the retina is solely dominated by the cone photoreceptors ${ }^{3}$ and MP is found on the Henle fiber layer of the cone axons $^{4}$ and outer segments of the rod photoreceptors. ${ }^{5}$

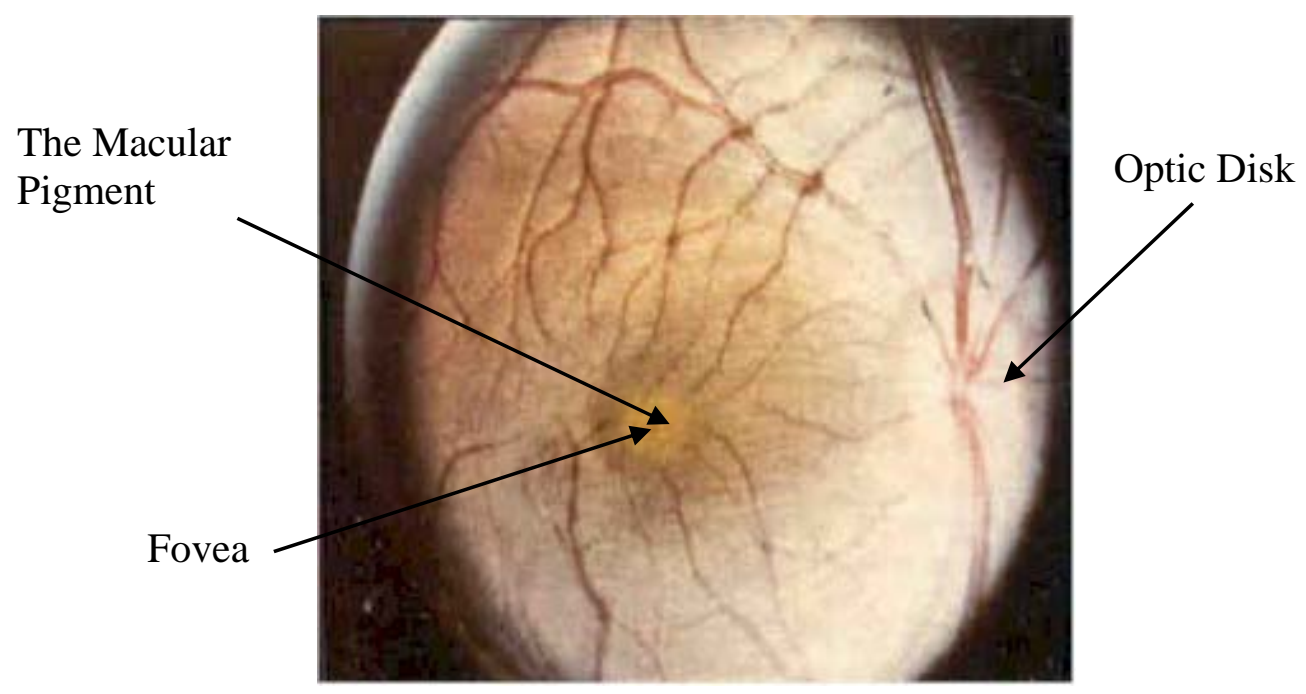

Fig.1.1 The location of the macular pigment in the macaque retina.Image courtesy: Fig 1(a) of Malinow et al (1980). ${ }^{6}$

Entoptic phenomena such as the observation of Haidinger brushes ${ }^{4,7}$ and Maxwell's spot $^{8}$ are explained due to the presence of MP around the fovea. The phenomenon of Haidinger brushes can be observed as a dark shape in the form of a "bow tie" by an individual when gazing at a uniformly illuminated surface 
through a plane polarizer. ${ }^{9}$ The pattern rotates if the polarizer is rotated. The rotation of the polarizer increases its visibility. The observation of the Haidinger brushes is based on the dichroic property of the component molecules of the MP, ${ }^{9}$ the molecules whose axis lie in the plane same as the plane of polarization of the incident light absorb the light producing the “dark” bow tie like feature as viewed

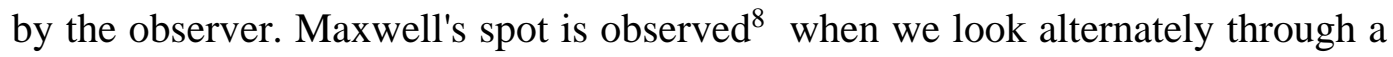
yellow and a blue color filter. A dark region in the form of a circle is seen around the area of gaze fixation when looking through the blue filter. ${ }^{10}$ The fixation of gaze being directly mapped to the fovea centralis and MP being mostly concentrated in the foveal region with its preferential absorption in the blue end of the spectrum is the explanation for this effect.

Macular pigment is composed primarily of the carotenoids lutein and zeaxanthin. ${ }^{11,12}$ The distribution of the component carotenoid molecules across the retina was first published by Bone et al. in $1988 .{ }^{13}$ Some of the lutein is converted into meso-zeaxanthin in the central region of the retina. Therefore, in the foveal region, the concentration level of meso-zeaxanthin reaches maximum, and is at negligible levels in the periphery of the retina. The distribution profile of MP along with its component molecules are shown in Fig. 1.2 next page. 


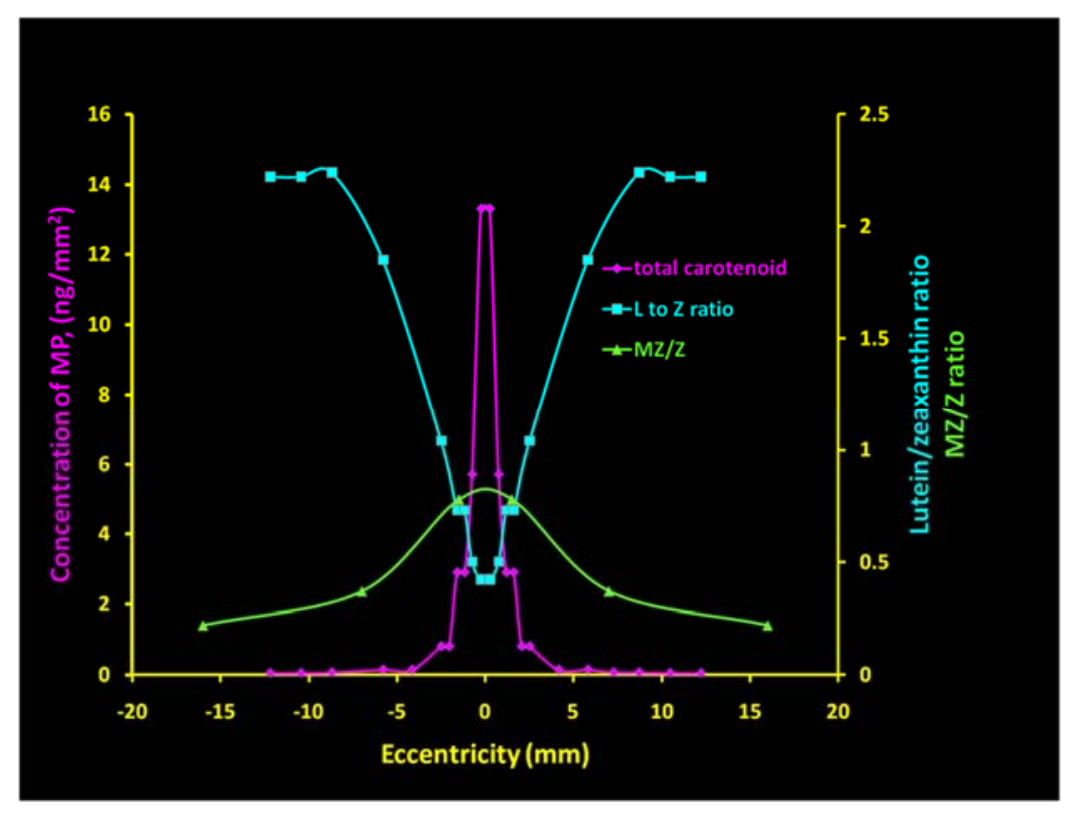

Fig. 1.2 The spatial distribution of the total carotenoid or the macular pigment in the retina is shown by the purple line. The cyan line shows the ratio of lutein to zeaxanthin. In the central retina zeaxanthin is the dominant carotenoid and the ratio of meso-zeaxanthin to zeaxanthin is shown by the green line. Image courtesy : Bone et al 1988. ${ }^{13}$

\section{Section 1.2}

\section{Absorbance spectrum of the Macular Pigment}

Wald in 1945 published the optical density spectrum of MP. ${ }^{14}$ Bone et al. ${ }^{15}$ applied heterochromatic flicker photometry (HFP) to 49 individuals to measure the macular pigment optical density (MPOD), and their results agreed with that of Wald's published work in 1945 . However a precise optical density spectrum was published by Bone in $1992^{9}$ where the density spectrum of MP, derived from its dichroic properties, appeared almost identical to the similar spectrum obtained from liposome-bound zeaxanthin and lutein in an environment duplicating that associated with the MP in the retina. The normalized optical density spectrum of 
MP is shown in Fig. 1.3. Also, data gathered by Bone in 1971 had shown that there is a wide variability in the amount of pigment possessed by individuals, suggesting diet, genetic, and environmental factors can be responsible for such variability. ${ }^{16}$ Further it has been also shown that diets rich in carotenoids or supplements containing the component carotenoids of the macular pigment can help in improving the level or concentration of MP in an individual's retina. ${ }^{17}$

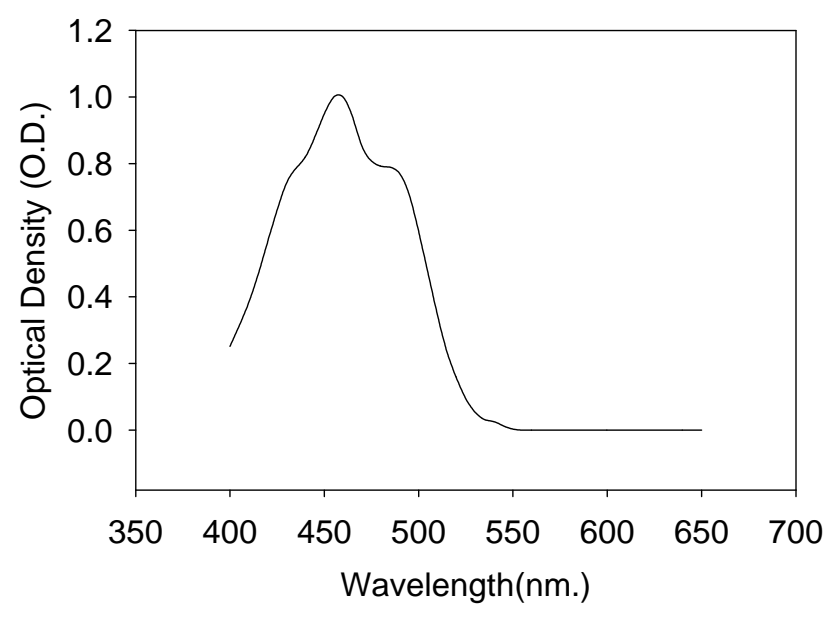

Fig1.3 The normalized optical density spectrum of the macular pigment, obtained from liposome-bound zeaxanthin and lutein as published in Bone et al in 1992. ${ }^{9}$

\section{Section 1.3}

\section{Macular Pigment in relation to proper eye health}

The fovea is dominated by the cone photoreceptors, which are responsible for vision under photopic or bright light conditions ${ }^{18}$ and vision demanding high levels of acuity. ${ }^{18}$ Therefore to preserve good vision standards, maintenance of cone health is of utmost importance. 
There are various reasons responsible for changes in the retina leading to loss of vision, but one of the conditions that has been found to have a predominant statistical importance, especially amongst the elderly population, is age-related macular degeneration (AMD) ${ }^{19}$

Age related macular degeneration is associated with the loss of central vision. An example of the appearance of the field of view to an individual with AMD is shown in Fig.1.4.b.

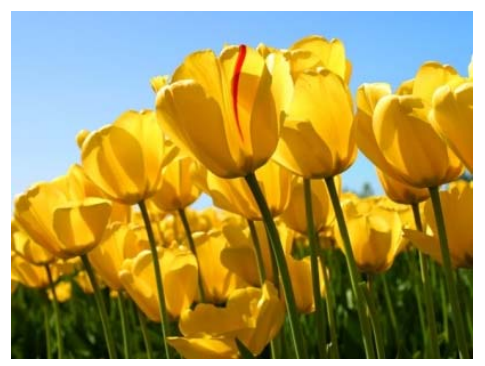

Fig.1.4 (a) Sample field as seen by a person with no vision anomaly.

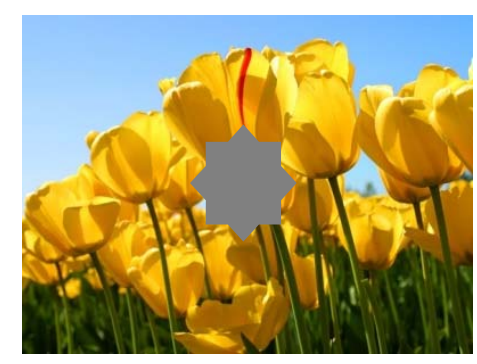

Fig.1.4 (b) Sample field as seen a person suffering from AMD.

Vision loss as a result of AMD is classified into two types viz. dry and wet. ${ }^{20}$ Degeneration in visual standards in the foveal region associated with the accumulation of drusen or cellular debris between the retinal pigment epithelium and the choroid is classified as dry AMD. ${ }^{20}$ Wet AMD on the other hand is associated with neovascularization of the aging retina, which involves formation of blood vessels in the macular region. These newly formed blood vessels being weak are associated with leakage leading to the loss of central vision.

Amongst the various risk factors ${ }^{21}$ for developing AMD, photooxidative ${ }^{22}$ damage to the photoreceptors has been cited as an important one. Light of shorter 
wavelength, especially in the blue region of the spectrum, aids in transforming the available oxygen molecules in the retina ${ }^{23}$ to reactive oxygen species such as singlet oxygen. The subsequent interaction of these oxygen species with the photoreceptor cells in the retina results in photooxidative damage of the photoreceptors. ${ }^{24}$

As the MPOD spectrum in Fig.1.3 is seen to be of highest absorbance at around $460 \mathrm{~nm}$ and decreasing to almost zero at $550 \mathrm{~nm}$, such a characteristic of the absorbance spectrum of the MP is understood to impart it the capability of acting in a protective capacity against excessive photooxidative damage to the photoreceptors. ${ }^{25,26}$ The identification of this protective role of the MP against photooxidative stress contributing to the damage to the photoreceptor cells, has made it an important subject of research in modern days. Further, it has been established that the higher the concentration of this pigment in an individual's retina, the better he/she might be protected against development of macular degeneration. ${ }^{27,28}$ As the levels of MPOD can be elevated only by diet or by consumption of supplements containing the component carotenoids, measurement and monitoring levels of MPOD is essential so that individuals can be prescribed the necessary preventive measures.

Cataract, ${ }^{29}$ along with AMD, is also a vision impairment condition, which instead is a consequence of a heavily opaque lens and primarily treated surgically. ${ }^{29}$ However, once an individual undergoes treatment for removal of the opacity associated with the lens, he/she is more susceptible to retinal exposure to light in 
the blue region of the spectrum. This therefore increases the chance of photooxidative damage to the photoreceptors and the development of AMD. ${ }^{30}$ Therefore clinical practitioners, prior to removal of cataract, should consider measuring the MPOD levels as a precautionary step and, if necessary, recommending individuals to modify their diet or take supplements in order to elevate protection against blue light damage by increasing the level of MP in the patient's retina. Hence, measurement and monitoring of MPOD of the patient regularly is important.$^{31}$

Such measurements, ideally done under clinical settings, therefore prioritize development of repeatable, reliable, and non-invasive measurement techniques and instruments.

\section{Section 1.4}

\section{Methods and techniques for measuring levels of Macular Pigment}

Levels of MP possessed by an individual, obtained by in vivo methods, are either in terms of optical density ${ }^{27}$, or are in the form of signal counts available from

resonance Raman spectroscopy. ${ }^{32}$ Over the years researchers have developed various techniques for measuring macular pigment optical densities (MPOD) which are either signal-based such as the autofluorescence ${ }^{33}$ and reflectance ${ }^{34}$ measurement techniques, or are psychophysical in nature such as heterochromatic flicker photometry (HFP) ${ }^{35,36}$ and heterochromatic brightness matching (HBM). ${ }^{37}$ 
The signal-based autofluorescence and the reflectance method, aside from being expensive, suffer from loss of signal in the aging eye. In the case of autofluorescence, such loss is the result of scattering of light ${ }^{38}$ by the aging lens and for reflectance measurements, losses in signal are caused by the presence of drusen in the back of the retina. ${ }^{38,39}$

Signal loss is not a considerable factor in psychophysical methods. The psychophysical methods also involve minimal risk, are subject-friendly and instrumentation is relatively easy to fabricate. On the basis of the factors mentioned, the psychophysical techniques have become the preferred choice of MPOD measurement amongst researchers and clinical practitioners. Between HBM and HFP, HFP has emerged as the more popular choice of psychophysical methods of measurement. The reason for HFP to become the preferred choice has been the strong similarity of the MPOD spectrum measured in vivo by HFP to that measured under in vitro conditions. ${ }^{35,15}$

Though researchers have pursued refinements of HFP,,$^{40,41,42,35}$ at the heart of HFP measurement is the concept of achieving a luminance match between two light sources made to flicker in anti-phase to each other ${ }^{32}$. Through instrumentation techniques, the frequency of flicker can be controlled by adjusting the angular frequency of a rotating chopper as described in Bone and Landrum ${ }^{32}$ or electronically as described in Bone and Mukherjee. ${ }^{43}$ To facilitate measurement, one of the component light sources of the stimulus has a peak wavelength close to the peak absorbance of the MP and the other with a peak at a region of the 
spectrum where the absorbance of MP is almost zero. ${ }^{44}$ Such sources can be derived from the light emitted by a single Halogen lamp and passing the light through interference filters to obtain the desired wavelengths, ${ }^{35}$ or from independent LED sources ${ }^{43}$. Heterochromatic Flicker Photometers that derive light solely from LED sources are termed as LED-based machines. The perception of flicker is minimized by either the operator or the subject, adjusting the intensity of the light in the blue channel either by translating a variable density wedge ${ }^{35}$ for halogen-based instruments or by adjusting the settings of a logarithmic potentiometer ${ }^{40}$ in LED-based instruments.

As the MP is primarily concentrated in the fovea, with its distribution becoming almost negligible beyond $5^{\circ}$ eccentricity in the retina, ${ }^{45}$ HFP measurement is divided into two phases, foveal and perifoveal. Testing for the foveal phase involves achieving a minimum flicker or no flicker for a stimulus that is entirely imaged on the fovea of the retina. Such a stimulus is generally of the order of 1 to 1.5 degrees in diameter. ${ }^{35}$ As a result of the spatial distribution profile of the MP, the elimination or minimization of flicker for the entire stimulus is affected by the presence of MP along with other pre-receptoral filters, chiefly the lens in the eye. $^{46,47}$

For the foveal measurements, the subject is directed to keep his/her gaze fixed at the center of the presented stimulus and to adjust a handheld control to achieve a flicker null. For subjects who find difficult the simultaneous operation of the control and keeping their gaze fixated, there is the provision in some instruments 
for the operator to adjust the settings. ${ }^{39}$ Such a provision necessitates the subject to communicate to the operator verbally whether a flicker null is achieved or not. For statistical purposes, the flicker null/minimum settings are recorded a minimum of three times, but more usually, five times.

On completion of the foveal phase, the perifoveal phase of the testing procedure is conducted. For the perifoveal flicker null testing, some instruments require the subject to fixate their gaze at a different location in the field of view and minimize the flicker for the previously used foveal stimulus, which at this time appears to be imaged in the perifoveal region of the retina. However some instruments have an alternative approach to the above mentioned procedure. The alternative approach associated with perifoveal testing is minimizing or adjusting for a flicker null condition in the periphery of a larger centrally viewed stimulus either in the form of a full circle as used in the instrument described by Bone et al. ${ }^{43}$ or an annulus as described in Wooten et $a l .{ }^{41}$ which replaces the previously tested smaller stimulus. The large stimulus replacing the smaller stimulus is typically of the order of $15^{\circ}$ diameter in size. Regardless of the type of stimulus used, the flicker null settings reported can be attributed to the presence of pre-receptoral filters aside from MP, primarily the lens. ${ }^{43}$ Similar to the foveal measurements, the measurements are repeated at least three times. The difference between the intensity settings for the foveal and the perifoveal flicker null settings can hence be considered to be a direct consequence of the presence of MP. Therefore, applying the necessary mathematical calculations and taking into account various 
spectral templates, we can compute the luminance associated with the individual light sources at the flicker null/minimum settings and calculate the MPOD.

Though significant refinements have been achieved over the years in MPOD measurement by HFP, there are still two drawbacks that have not yet been eliminated. One such drawback is Troxler fading of a peripherally viewed small stimulus. $^{43}$ The other occurs if the subject, during the perifoveal testing, inadvertently looks directly at the flickering stimulus instead of the eccentric fixation mark while reporting the flicker null condition. In such cases, he/she would merely be repeating the foveal phase of the testing procedure. ${ }^{43}$

The Mapcatsf instrument addresses the two above mentioned difficulties encountered in other HFP instruments. In the present dissertation we evaluate the suitability of using Mapcatsf, a LED-based, fully microprocessor-controlled HFP instrument whose primary features are to measure MPOD and the lens density in terms of lens equivalent age, by clinical practitioners and researchers. 


\section{Chapter 2}

\section{Instrument Design and Subject Testing Procedure}

\section{Section 2.1}

\section{Instrument design}

The MPOD measurement by HFP is a two-step process and the reason for the limits or size of the stimulus used in each of the separate steps is explained referring to Fig. 2.1 below.

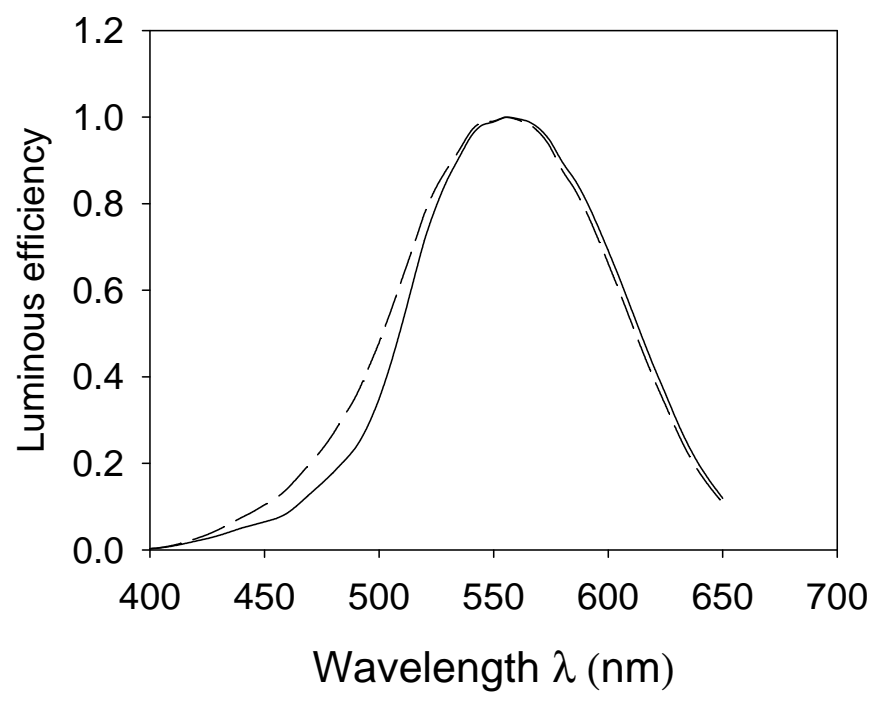

Fig 2.1 The luminous efficiency functions plotted for centrally viewed fields of 10 degree (dashed line) and 2 degree (solid line) in diameter.

In Fig. 2.1, the difference in the photopic luminous efficiency functions for $2^{\circ}$ and $10^{\circ}$ stimuli is seen in the short wavelength region. Such a difference is attributed to the concentration of MP primarily in and around the foveal region. Therefore 
for the foveal phase of the flicker null testing in most of the instruments, a small centrally viewed stimulus of around 1 to 2 degree diameter is used. Such a stimulus when imaged on the retina falls directly on the fovea. The stimulus used in Mapcatsf for foveal testing is $1.5^{\circ}$ in diameter and is associated with a vertical/horizontal cross hair for central gaze fixation. Gaze fixation is associated with eye movements, ${ }^{48,49}$ which are termed as fixational eye movements, a topic discussed in detail in Chapter 9. The mean amplitude associated with such eye movements is of the order of $0.36^{\circ} .^{50}$ Therefore the area of retina sampled during MPOD measurement could be larger than the stimulus area depending on the size of these eye movements. To minimize this effect, the size of the foveal stimulus was chosen to be of $1.5^{\circ}$ diameter rather than $1^{\circ}$ as in a number of other instruments.

For the perifoveal testing on Mapcatsf, a large centrally viewed $15^{\circ}$ diameter circular stimulus associated with vertical/horizontal cross hairs for gaze fixation is used. As the concentration of MP reduces to negligible levels at about $5^{45}$ to $7^{51}$ degrees eccentricity in the retina, the 10 degree luminous efficiency function is applicable for the $15^{\circ}$ diameter stimulus without any significant differences being associated. The flicker null reported in the periphery of such a large stimulus can be considered to have no association with the absorbance of MP, ${ }^{45}$ but rather to be primarily affected by the optical density of the lens. ${ }^{43}$

The schematic optical setup of Mapcat is shown in Fig. 2.2. The LED light sources used to generate the stimuli are a 3W blue LED of peak wavelength at 
$455 \mathrm{~nm}$ and half bandwidth $20 \mathrm{~nm}$ and a $1 \mathrm{~W}$ green LED of peak wavelength at $515 \mathrm{~nm}$ and half bandwidth of $33 \mathrm{~nm} \cdot{ }^{43}$ The LED sources associated with heat sinks are mounted in the integrating sphere $\mathrm{S}_{1}$, so that the resultant light obtained is spatially uniform.

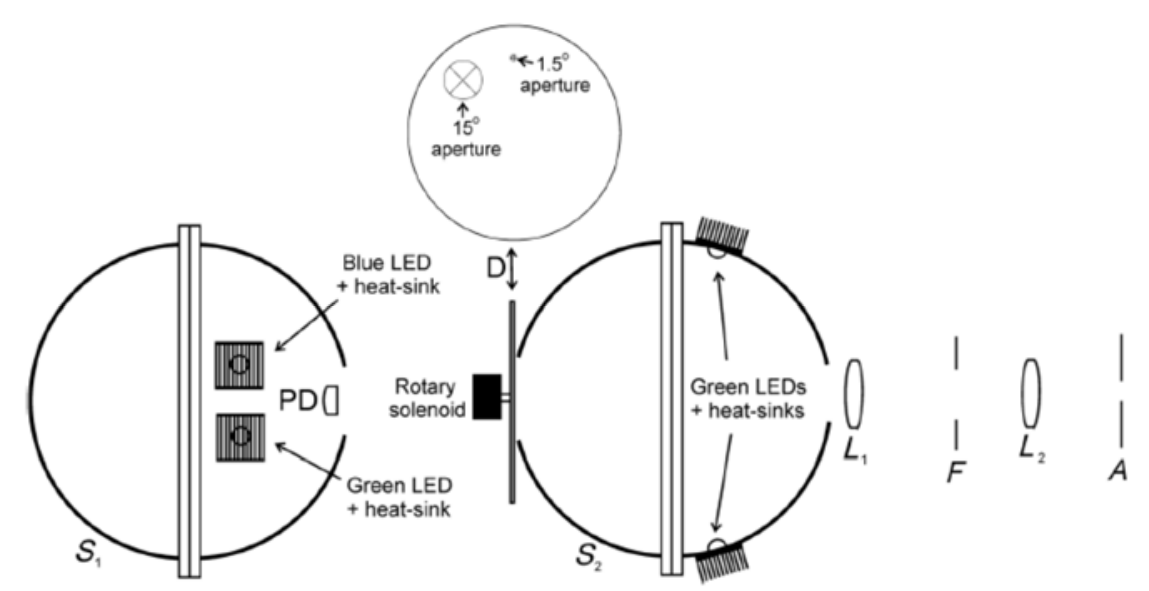

Fig 2.2 The optical setup of Mapcatsf. The interior of the sphere $S_{1}$ is illuminated by the blue and green LEDs. The intensity settings of the blue and green LEDs at flicker null are measured by the photodioide PD. The apertures on disk D provide the $1.5^{\circ}$ and $15^{\circ}$ stimuli as viewed by the telescopic setup which consists of the lenses $\mathrm{L}_{1}$ acting as the objective and $\mathrm{L}_{2}$ acting as the eyepiece along with the field stop $\mathrm{F}$ and aperture $\mathrm{A}$. The front surface of $\mathrm{D}$ is illuminated by the LEDs on the sphere $\mathrm{S}_{2}$. Image courtesy from SPIE, $\mathrm{JBO}^{43}$

It is known that current-controlled LED outputs result in a shift of their peak wavelength with current. ${ }^{43}$ To avoid such a change in the LED spectrum, the outputs were frequency controlled. Such a control mechanism was obtained by applying frequency-modulated fixed amplitude pulses to the LEDs. Bi-stable electronic circuits were used to obtain the "on" and "off" cycles, so as to enable the output of the blue and the green LEDs to be in antiphase to each other at frequencies between 10 and $40 \mathrm{~Hz}^{43}$ resulting in the visual perception of flicker. A logarithmic potentiometer, in the form of a handheld control device, is used to 
adjust the intensity of the blue LED source by the subject to report a flicker null condition while centrally viewing the testing stimulus.

A flicker null condition is achieved when the photoreceptors in the subject's retina perceive matching luminance levels between the light in the blue channel and light in the green channel. A photodetector positioned on the sphere $S_{1}$ as shown, samples the light from the inner rear surface of the sphere at the flicker null condition. The microprocessor associated with the photodetector is programmed to gather the intensities recorded by a sample-and-hold technique. Such a technique gathers many samples of the intensities of the light from both the blue and the green LEDs. For computational purposes; the averages of the results are taken and are represented by the symbol $\phi$, with pertinent subscripts for the blue and the green LEDs respectively, shown in Eq.s (1) - (3) in section 2.2 .

The emergent, spatially uniform light from sphere $S_{1}$ is designed to pass through additional apertures provided on an opaque disk " $\mathrm{D}$ " which, along with the telescopic setup consisting of the lenses $\mathrm{L}_{1}$ and $\mathrm{L}_{2}$, aid in setting the desired dimensions of $1.5^{\circ}$ and $15^{\circ}$ diameter for the circular stimuli used during the foveal and the perifoveal testing phases. Further, the field stop "F" limits the total field size at 24 degrees. ${ }^{43}$ The disk $\mathrm{D}$ is rotated to switch stimuli between the two phases of testing by a rotary solenoid. To facilitate gaze fixation, both the stimuli used are provided with cross hairs. 
Just anterior to the disk $\mathrm{D}$, a second sphere labeled as $\mathrm{S}_{2}$, is located, fabricated out of two hemispherical surfaces. Two 1W green LEDs of the same peak wavelength and bandwidth as the ones in sphere $\mathrm{S}_{1}$ are mounted on $\mathrm{S}_{2}$. These LEDs are also provided with heat sinks and are used to illuminate the front surface of the disk D in order to create the necessary photopic viewing conditions. To facilitate the condition of uniform luminance at flicker null settings across both the field and the stimulus, the luminance of the green LEDs in the sphere $S_{2}$ is set to match the luminance of the green component of the stimulus. The interior surface of the hemisphere closest to the disk D is painted white and that of the other hemisphere is painted black, both with a matte finish. The matte black finish prevents feedback of any scattered light generated in $S_{2}$ to the reflecting surface of the sphere $\mathrm{S}_{1}$.

Aside from the lens $\mathrm{L}_{2}$ and the viewing aperture "A", the rest of the setup is mounted on a translational slide controlled by a stepper motor. Such a mechanism is provided so that distinct focusing of the cross hair stimulus on the disk " $\mathrm{D}$ " is facilitated according to the participating subject's visual accommodation level. As maintaining the angular size of the stimuli during the testing phases is very crucial, the lens $\mathrm{L}_{2}$ is positioned at a distance from the viewing aperture A such that the distance between the eyepiece lens $\mathrm{L}_{2}$ and the nodal point of the subject's eye is equal to the focal length of $\mathrm{L}_{2}$. The particular alignment of the lens $\mathrm{L}_{2}$ described results in a constant angular size of the retinal image, regardless of the subject's state of accommodation. 


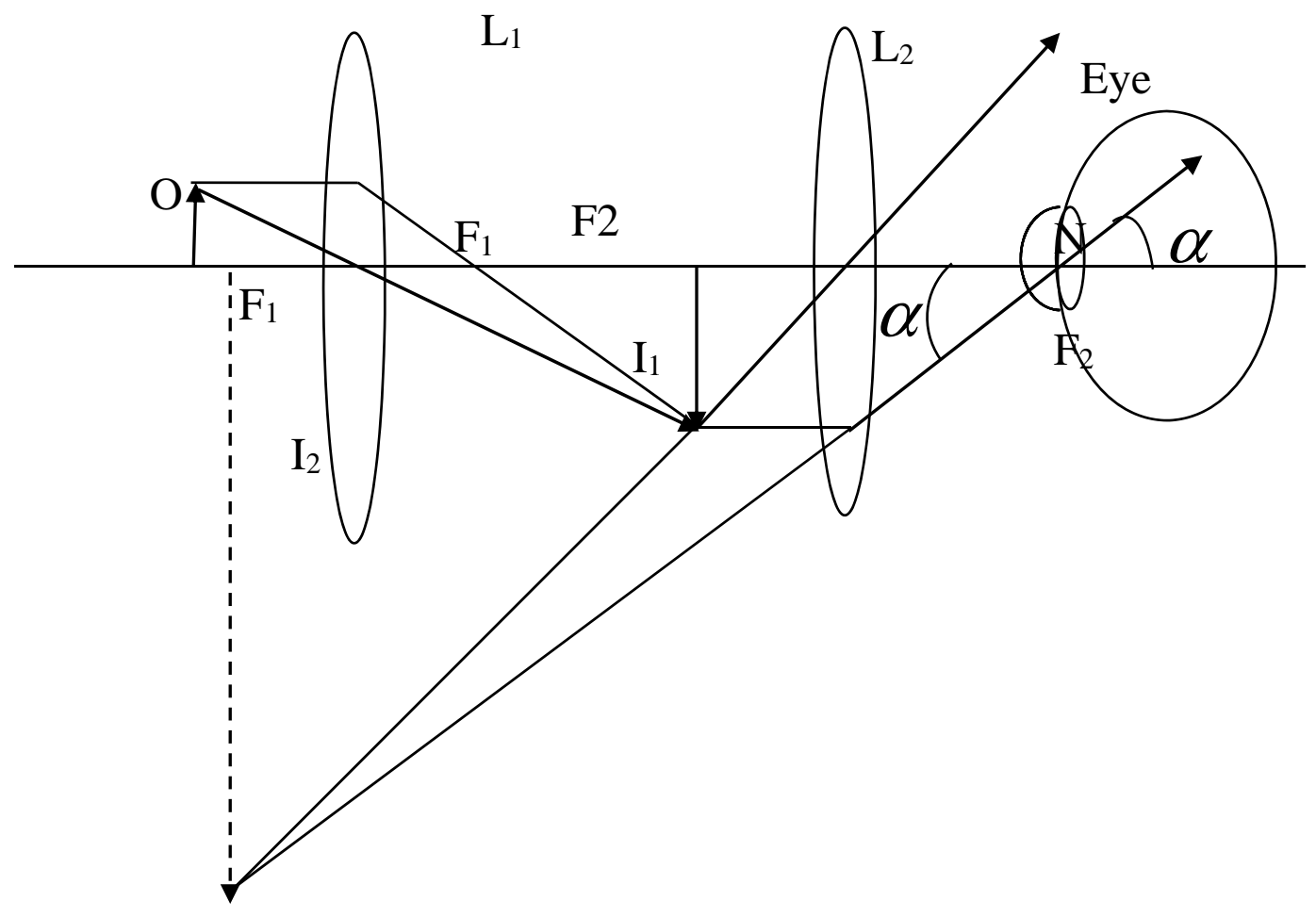

Fig. 2.3 The ray diagram explains the fact that when the distance between the nodal point of the eye and the eyepiece lens $\mathrm{L}_{2}$ is equal to the focal length of $\mathrm{L}_{2}$, the angle subtended by the stimulus imaged on the retina is the same regardless of the position of the second image $\mathrm{I}_{2}$, which is adjusted based on the subject's accommodation.

In Fig. 2.3, F1, F2 are the focal points of the objective and the eyepiece lenses $\mathrm{L}_{1}$, $\mathrm{L}_{2}$ respectively, with " $\mathrm{N}$ " being the nodal point of the eye. "O" depicts the extremity of the stimulus used, with $I_{1}$ and $I_{2}$ being the images formed by the lenses $L_{1}$ and $L_{2}$. Since $I_{2}$ is a virtual image, it is represented by a dashed line in the Fig.. The angle " $\alpha$ ” depicts the angular size of the image of $\mathrm{I}_{2}$ formed on the retina. Applying trigonometry we get:

$$
\tan \alpha=\frac{h_{I_{1}}}{F_{2}}
$$


In Eq. 1 above $h_{I_{1}}$ is the height of the image $\mathrm{I}_{1}$ and $F_{2}$ is the focal length of the lens $\mathrm{L}_{2}$. By applying the lens Eq. to the lens $\mathrm{L}_{1}$, the radii of the original apertures on disk " $D$ " can be fabricated for the $1.5^{\circ}$ and $15^{\circ}$ stimuli for foveal and perifoveal testing respectively.

Since Mapcatsf does not employ a dental bite, lack of precise eye positioning could result in small variations in the distance between the nodal point of the eye and the eyepiece lens, but errors introduced thereby due to the unaligned eye positioning can be ignored. ${ }^{43}$

\section{Section 2.2.1}

\section{Theory for MPOD measurement}

The photodetector in the sphere $S_{1}$ is used to detect the intensity of the green LED, which is steady, and the adjusted intensity of the blue LED at the flicker null condition. The photodetector outputs recorded for the blue and the green LEDs at the settings of foveal and perifoveal flicker null can be expressed mathematically as shown below:

$$
\begin{aligned}
& \phi_{B F}=k_{F} \int I_{B}(\lambda) S(\lambda) d \lambda \\
& \phi_{B P}=k_{P} \int I_{B}(\lambda) S(\lambda) d \lambda \\
& \phi_{G}=\int I_{G}(\lambda) S(\lambda) d \lambda \ldots .
\end{aligned}
$$


The terms $I_{B}, I_{G}$ are the normalized intensity spectra of the blue and the green LEDs. The multipliers $k_{F}, k_{P}$ are the factors that arise due to adjustment of the luminance levels of the blue LED by the subject to arrive at the flicker null/minimum conditions during the foveal and the perifoveal phases of testing respectively. The term $S(\lambda)$ is the sensitivity spectrum of the photodetector mounted in the sphere S1. From Eq.s (2) and (3)

$$
\frac{k_{F}}{k_{P}}=\frac{\phi_{B F}}{\phi_{B P}} \ldots \ldots \ldots \ldots \ldots \ldots \ldots
$$

The achievement of the flicker null during the foveal or the perifoveal phase is the result of matching of luminance levels between the light from the component sources at the foveal region of the retina during the foveal phase or at the periphery during the perifoveal phase. The luminance match conditions for the foveal and the perifoveal flicker null/minima conditions are expressed mathematically as shown in Eqs. (6) and (7) respectively.

$$
\begin{aligned}
& k_{F} \int I_{B}(\lambda) 10^{-P D(\lambda)} V(\lambda, a) d \lambda=\int I_{G}(\lambda) 10^{-P D(\lambda)} V(\lambda, a) d \lambda \\
& k_{P} \int I_{B}(\lambda) V(\lambda, a) d \lambda=\int I_{G}(\lambda) V(\lambda, a) d \lambda
\end{aligned}
$$

In both the Eq.s, the term $V(\lambda, a)$ is the age-modified luminous efficiency function of the human eye, obtained for a centrally viewed stimulus of $10^{\circ}$ diameter. Though a standard $2^{\circ}$ luminous efficiency function is available, the $10^{\circ}$ function was used in the Eqs. so that the transmittance of MP could be observed 
as a separate parameter and the subject's peak MPOD can be computed from it. As the MPOD is considered to be almost negligible beyond 5 to 7 degrees eccentricity, ${ }^{51}$ the $15^{\circ}$ diameter stimulus was used for perifoveal testing. The term $D(\lambda)$ is the macular pigment optical density spectrum, ${ }^{9}$ normalized to unity at the peak optical density and $P$ is the peak MPOD of the subject being tested ${ }^{43}$. Taking into account the spatial distribution profile of $\mathrm{MP}$, the term representing the transmittance of MP is absent in Eq. (7). Division of Eq. (6) by Eq. (7), and taking into account the results from Eq. (5), leads to

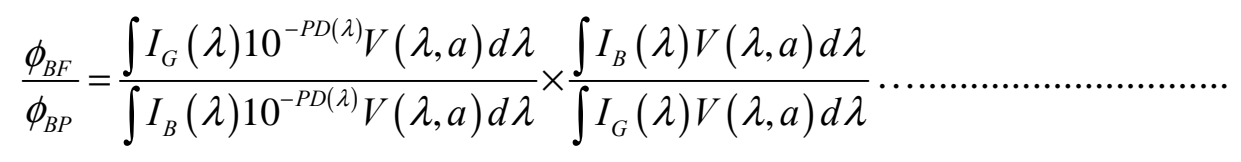

If instead of LED sources, monochromatic light sources were used, Eq. (8) above reduces to

$$
P=\log \left(\frac{\phi_{B F}}{\phi_{B P}}\right)
$$

Such an expression, however, is only valid if the density spectrum of MP $D(\lambda)$ has its maximum at the wavelength of the blue source and is zero at the wavelength of the green source.

On inspection of Eq. (8), if the spectral characteristics of the LED sources are known along with $V(\lambda, a)$ (the $10^{\circ}$ luminous efficiency function for a certain age), then we can numerically solve Eq. (8) to obtain a relation between $P$ and the 
ratio of the photodetector outputs of the blue light intensities recorded at the foveal and the perifoveal flicker null conditions, $\frac{\phi_{B F}}{\phi_{B P}}$.

Sagawa and Takahashi ${ }^{52}$ published a template as shown below in eq. (10) which describes the variation in the luminous efficiency function with age. The reference age has been changed from the original 64.9 years to 32 years since the CIE 1951 approved $V_{10}(\lambda)$ was the fitted data for a group of subjects whose average age was 32 years.

$$
\log V(\lambda, a)=\log V(\lambda, 32)+(a-32) \alpha(\lambda) \ldots \ldots \ldots \ldots \ldots \ldots \ldots(10)^{52}
$$

The parameter $\alpha_{\lambda}$ is the change per year of the log luminous efficiency, attributed to the aging lens by Sagawa and Takahashi. Hence the parameter “ $a$ ” associated with $V(\lambda)$ can be termed as the "lens equivalent age" or LEA.

In Fig. 2.4 the plot of “ $P$ ” versus $\log \left(\frac{\phi_{B F}}{\phi_{B P}}\right)$ is shown for various values of LEA. The LEA is computed from the perifoveal flicker null settings which we explain in the following section. Once the LEA is obtained, the MPOD \pm SEM (standard error in the mean) is computed by the microprocessor essentially by selecting the pertinent curve from Fig. 2.4. 


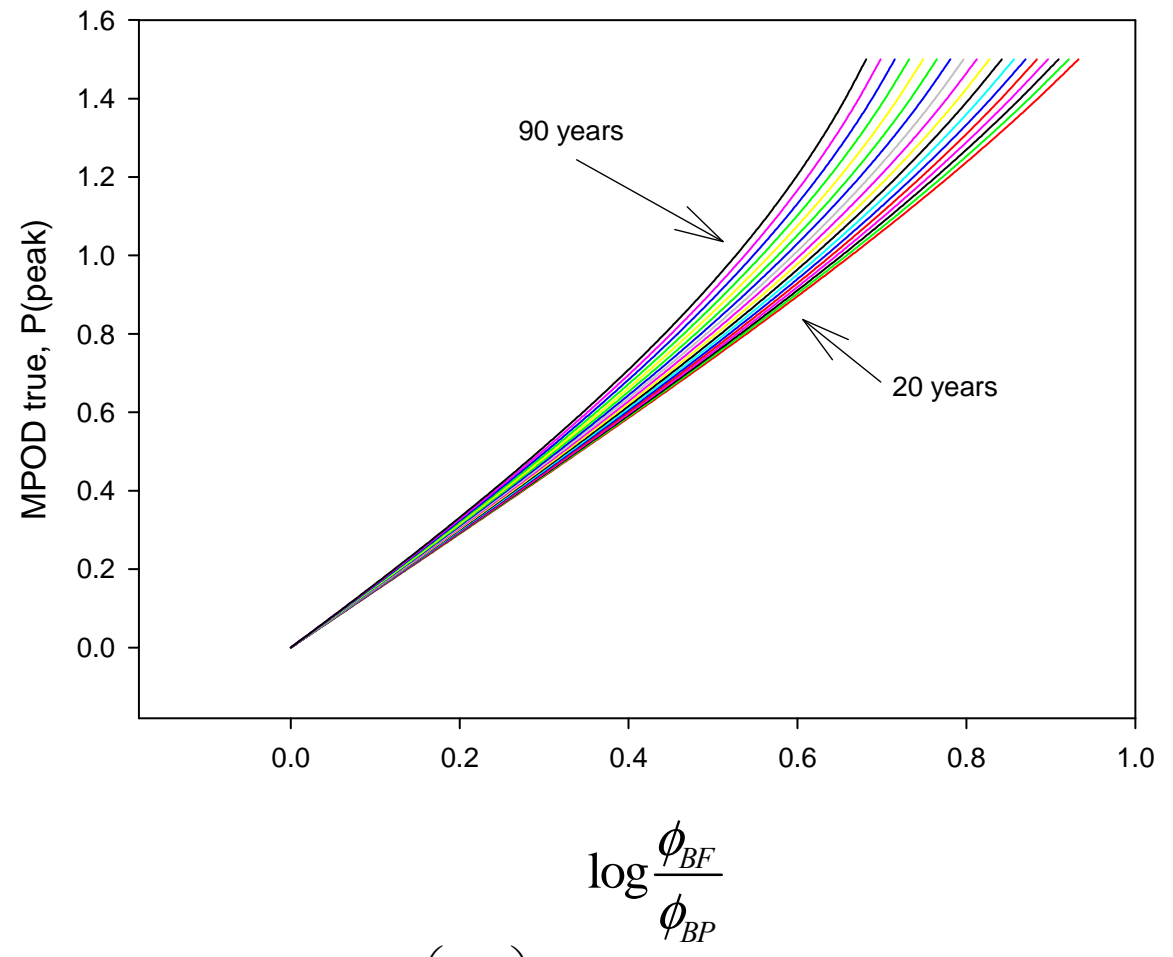

Fig. 2.4 The plots of $P$ vs $\log \left(\frac{\phi_{B F}}{\phi_{B P}}\right)$ for LEAs at 5 years intervals between 20 and 90 years.

Aside from computing the MPOD along with the associated standard error in the mean, the microprocessor computes the average percentage of the light blocked in the blue end of the spectrum (400 to $500 \mathrm{~nm}$ ) by computing the mean of the expression $\left(1-10^{-P D(\lambda)}\right)$. The capacity of Mapcatsf to compute the percentage of the light being blocked in the blue region of the visible spectrum is a more readily understood quantity for the layperson who is likely to be unfamiliar with the concept of optical density. 


\section{Section 2.2.2}

\section{Theory of LOD or LEA measurement}

At large retinal eccentricities, the presence of MP can be ignored. Taking into account the optical density spectrum of the lens, the perifoveal flicker null condition that result from match of luminances between light from the component blue and the green sources of the $15^{\circ}$ stimulus can be considered to be affected by the age-related changing levels of the density of the lens. The ratio of the photodetector outputs of the intensities of the blue and the green LEDs can be combined with luminance of the light sources at the perifoveal flicker null condition according to eq. (11) below.

$$
\frac{\phi_{B P}}{\phi_{G}}=\frac{\int I_{G}(\lambda) V(\lambda, a) d \lambda}{\int I_{B}(\lambda) V(\lambda, a) d \lambda} \times \frac{\int I_{B}(\lambda) S(\lambda) d \lambda}{\int I_{G}(\lambda) S(\lambda) d \lambda}
$$

If the spectral characteristics of the concerned variables in the right-hand side of eq. (11) are known, then the left hand side, or the ratio of the photodetector outputs, can be seen to be only dependent on “ $a$ ”. As the variable " $a$ ” in Eq. (11) has been referred to as LEA earlier, in Fig. 2.5 we show the variation of LEA with $\frac{\phi_{B P}}{\phi_{G}}$.A curvefit polynomial programmed in the microprocessor computes the LEA along with the associated standard error in the mean. 


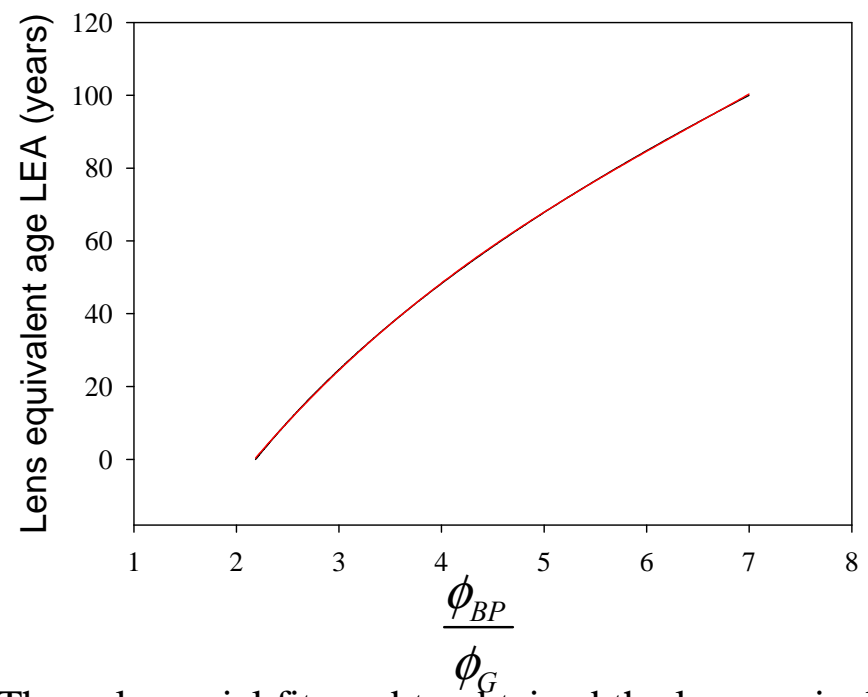

Fig. 2.5 The polynomial fit used to obtained the lens equivalent age or LEA from the intensity ratios of the blue and the green I.ED sources detected by the photodetector at perifoveal flicker null settings, $\frac{\phi_{B P}}{\phi_{G}}$.

\section{Section 2.2.3}

\section{LEA computation based on templates derived from excised lenses.}

Sagawa and Takahashi attributed the changes in the photopic luminous efficiency function in the shorter wavelengths to the age-related changes in the optical density of the lens. This prompted us to test templates developed from data from excised lenses, such as the ones by Pokorny et al. ${ }^{46}$ and van de Kraats and van Norren. ${ }^{47}$ To develop the luminous efficiency function, a 10-degree luminous efficiency function was obtained for an aphakic individual. To develop the luminous efficiency function for an aphakic individual the standard $V_{10}(\lambda)$ was divided by the transmittance of the lens for a 32 year old individual obtained from van de Kraat's age-related lens density template. The resulting function was subsequently multiplied by the age-dependent lens transmittance spectrum of the 
model being tested. The resultant $V_{10}(\lambda)$ was included in Eq. (10) to numerically solve for the ratio of the photodetector outputs $\frac{\phi_{B P}}{\phi_{G}}$ as a function of the lens equivalent age (LEA) labeled as " $a$ ". The graphical representation of the variation of $\frac{\phi_{B P}}{\phi_{G}}$ with LEA for the two templates tested, aside from the one by Sagawa and Takahashi, is shown in Fig. 2.6.

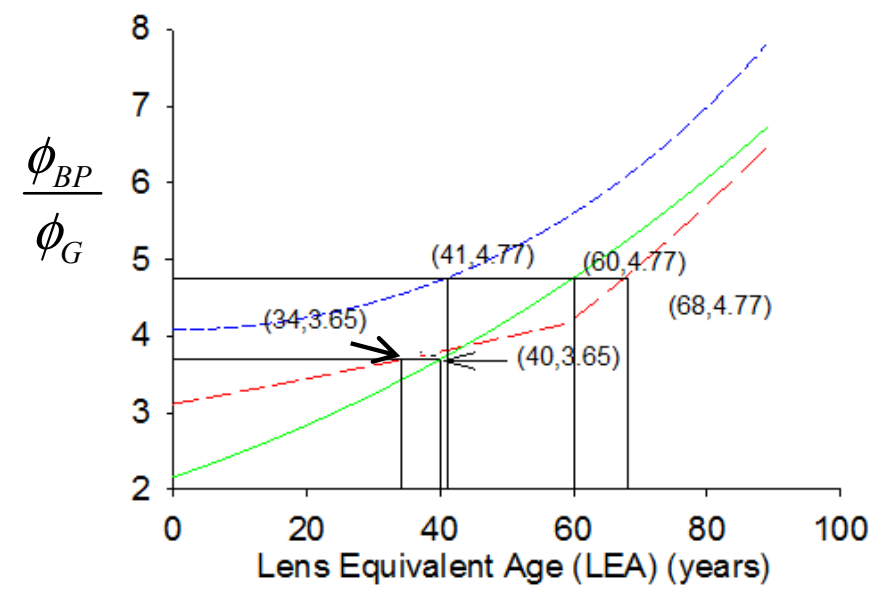

Fig. 2.6 The plots of $\frac{\phi_{B P}}{\phi_{G}}$ with LEA, using the three different templates.

The solid green line is obtained by using the template suggested by Sagawa and Takahashi. The red and blue dashed lines are obtained as a result of using templates suggested by Pokorny and van de Kraats respectively.

In Fig. 2.6 we observe that there are large discrepancies in the LEA measurements when comparing the ratio of the photodetector readings obtained using the three separate templates. We compare the results of the two templates taking the results 
derived from Sagawa and Takahashi as a standard. If, for example, an individual had an LEA of 40 years when using the Sagawa and Takahashi template, he/she would have an LEA of 34 if the Pokorny template was used and would not be traceable to any LEA if the van de Kraats template was used. Similarly if someone has an LEA of 60 years according to the Sagawa and Takahashi template, he /she would have an LEA of 41 years according to van de Kraat's template but would report an LEA of 68 years according to the Pokorny template. In chapter 3 we explain in detail why we programmed the Mapcatsf using the template by Sagawa and Takahashi.

\section{Section 2.3}

\section{Subject testing procedure:}

The testing on Mapcatsf enables testing one eye at a time. The instrument is placed on a hydraulic adjustable table with the subject seated on an adjustable chair to facilitate proper height adjustments. An adjustable chin rest is also provided to have the subject's head partially fixed. The non-testing eye is occluded by an eye patch. The occlusion of the non-testing eye was initiated on the basis of subjects' feedback, that if the non-testing eye was available for vision, it actually made gaze fixation difficult for the testing eye. Prior to the commencement of the testing, the entire test field consisting of the $1.5^{\circ}$ (foveal) central gaze fixating stimulus is focused based on the subject's accommodation level. During the focusing phase, the blue component and flicker are turned off leaving both the stimulus and the surrounding field uniform green. The handheld 
control device operates an optical encoder that provides movement of the translational stage via a stepper motor enabling back and forth movement of the optical layout aside from the eyepiece lens L2 and the viewing aperture "A”. Such a mechanism enables adjustment of the stimulus derived from the cross hair reticle on disk " $\mathrm{D}$ " so that it is focused on the retina according to subject's accommodation.

Once proper focusing has been ensured, the blue LED and the flicker are turned on by the operator. By default, the frequency is set to $24 \mathrm{~Hz}$ by the operator. At this stage, turning the knob controls the intensity of the blue LED, behaving as a logarithmic potentiometer. The operator guides the subject to turn the knob fully clockwise and then counter-clockwise to impart the idea of the change of the apparent flicker with the variation of intensity of the blue component of the stimulus. Once the subject gets accustomed to the operation involved in the testing, the subject is asked to fix his/her gaze at the center of the stimulus and operate the knob until a flicker null or minimum is visually perceived for the entire stimulus.

Further, the subject is also instructed that at the flicker null/minimum condition, the luminance of the surrounding green region will appear the same as that of the stimulus. The testing for the $1.5^{\circ}$ stimulus is called the foveal phase of the testing procedure since the entire stimulus is imaged on the foveal region of the retina. Once the setting for minimum or null flicker is achieved, the intensity of the blue light is recorded by the microprocessor and a subsequent offset is introduced in 
the intensity of the blue component of the stimulus. The process can be repeated a total of 10 times but is generally executed between 4 and 5 times. If a subject reports a wide range of knob settings for perception of flicker minimum, the frequency is reduced from $24 \mathrm{~Hz}$ and on the contrary if it gets hard to obtain a well-defined flicker minimum, the frequency is increased from $24 \mathrm{~Hz}$. The adjustment of the frequency based on the subject's performance is called "customized heterochromatic flicker photometry" or cHFP, first reported by Stringham et al..$^{53}$

On completion of the foveal phase, the $15^{\circ}$ diameter stimulus replaces the $1.5^{\circ}$ diameter stimulus and the flicker frequency is set at $31 \mathrm{~Hz}$ by default. For this phase of testing, the subject is directed to operate the handheld control and eliminate the flicker from the peripheral part of the stimulus while keeping his/her gaze fixed at the center of the cross hairs. Under such conditions, the subject observes a central flickering zone along with the flicker null zone in the periphery as shown in Fig. 2.7 below. 


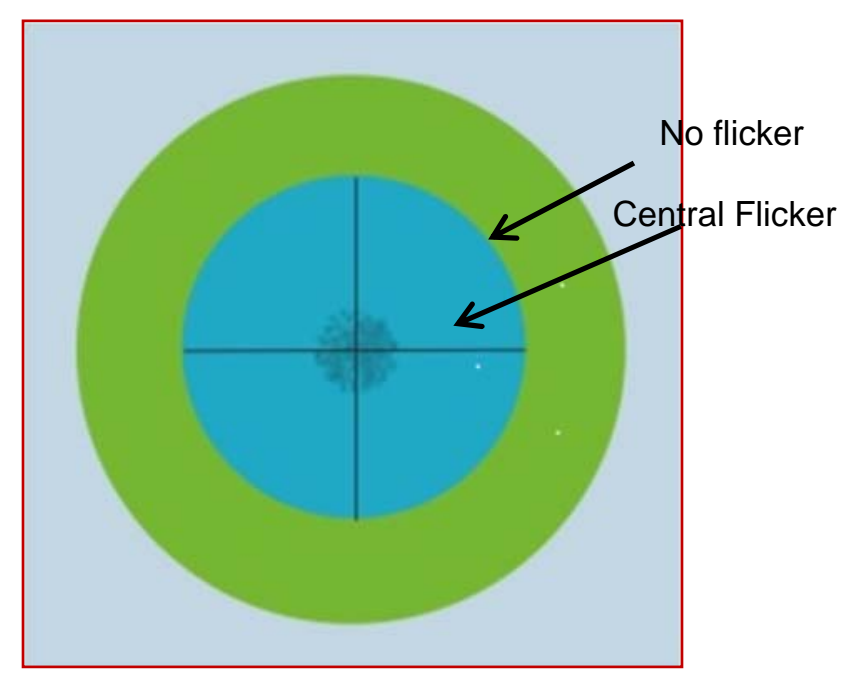

Fig 2.7 The appearance of the $15^{\circ}$ stimulus to the subject when the flicker null/minimum condition is reported in the periphery.

Further, it is communicated to the participating subject that the area of flicker null/minimum should be as large as possible leaving a central circular region of flicker around $\sim 5$ to 8 degree diameter. ${ }^{43}$ The flicker null condition is sharply defined when tiny offsets to the knob position from the flicker null settings result in emergence of flicker for the entire stimulus. As with the foveal phase, the measurements can be recorded 10 times but are generally limited to 4 or 5 times. The small flickering region surrounds the fixation point and is due to MP. Therefore maintenance of precise fixation is not necessary and, from experience, it has been observed that the phenomenon of Troxler fading is absent. Further, compared to another instrument, the Maculometer, ${ }^{54}$ the problem for some subjects of inadvertently gazing directly at a small perifoveal flickering stimulus is removed. The inadvertent shift in gaze to the perifoveal location is a prime 
concern since if the flicker null/minimum conditions are recorded when the subject is gazing directly at the intended perifoveal area, he/she is basically repeating the foveal phase of the testing since MP is mostly concentrated in the fovea.

On completion of the perifoveal measurements, the microprocessor computes the MPOD. Lens optical densities (LOD) are calculated from the perifoveal measurements. However the LOD is computed at $425 \mathrm{~nm}$ taking into account the age-related variation of luminous efficiency, as published by Sagawa and Takahashi. ${ }^{52}$ A tablet display attached to the outer cabinet of the instrument displays the MPOD, the LOD, and the lens equivalent age (LEA), along with the associated standard errors in the means of each of the computed quantities. As an additional and novel feature, the average percentage of blue light blocked by MP is also displayed along with the average intensities of the blue light detected at the foveal and perifoveal flicker null/minimum. The instrument, if interfaced by a USB cable, has the capacity to provide a printout of the results. A sample of a printed result is shown in Fig. 2.8 below. 


\section{MAPCATsf}

\subsection{DEGREE STIMULUS \\ 0.404 \\ 0.402 \\ 0.396 \\ 0.410 \\ 0.399}

\section{DEGREE STIMULUS}

0.272

0.271

0.276

0.261

Green Intensity $=0.071$

Macular Pigment Optical Density MPOD $=0.268+1-0.007$ Blue-light blocking by macular pigment $=35 \%$ Lens Optical Density $(425 \mathrm{~nm}) \mathrm{L}=0.603+/-0.011$ Effective age of lens $=31+/-1$

Foveal av $=0.402+1-0.002$

Perifoveal av $=0.270+1-0.003$

Fig 2.8 A printed sample of the test results. 


\section{Chapter 3}

\section{Pathway to Establishing Credibility of Mapcatsf}

\section{Section 3.1}

\section{Test Retest Results}

In order to establish the repeatability of measurements, we performed a test-retest analysis. Eighty three naïve subjects were recruited for this purpose. The subjects were all aged between 19 and 79 years (mean $\pm \mathrm{SD}=36 \pm 17$ years). Out of the 83 subjects, 4 had undergone cataract surgery. One had been diagnosed with dry AMD but still possessed a 20/20 level of visual acuity. The subjects, prior to being tested, signed an informed consent approved by the IRB at Florida International University. The study conformed to the tenets of the Declaration of Helsinki. The operator described every step of both the phases of testing to the subject using a Power-Point presentation. A minimum of 4 times, the flicker null was tested for both the foveal 1.5-deg stimulus and the perifoveal 15-deg stimulus. Both MP and the lens densities maintain almost the same level in both

the eyes, therefore only the right eye was tested. ${ }^{55,56}$ When questioned, the subjects responded that arriving at the flicker null settings for the 15-degree large stimulus was much easier than for the 1.5-deg stimulus, with Troxler fading not being an issue during the perifoveal phase of test. Such responses are supported by the average standard deviations for the foveal and the perifoveal flicker null settings which were 0.0263 and 0.0124 (arbitrary intensity units) respectively.

The retest was conducted on the same day following the completion of the test. To analyze repeatability, we conducted a Bland Altman analysis for the 83 
subjects by plotting the difference in the test-retest MPOD measurements with respect to the mean of the test-retest measurement. The plot is shown in Fig 3.1.

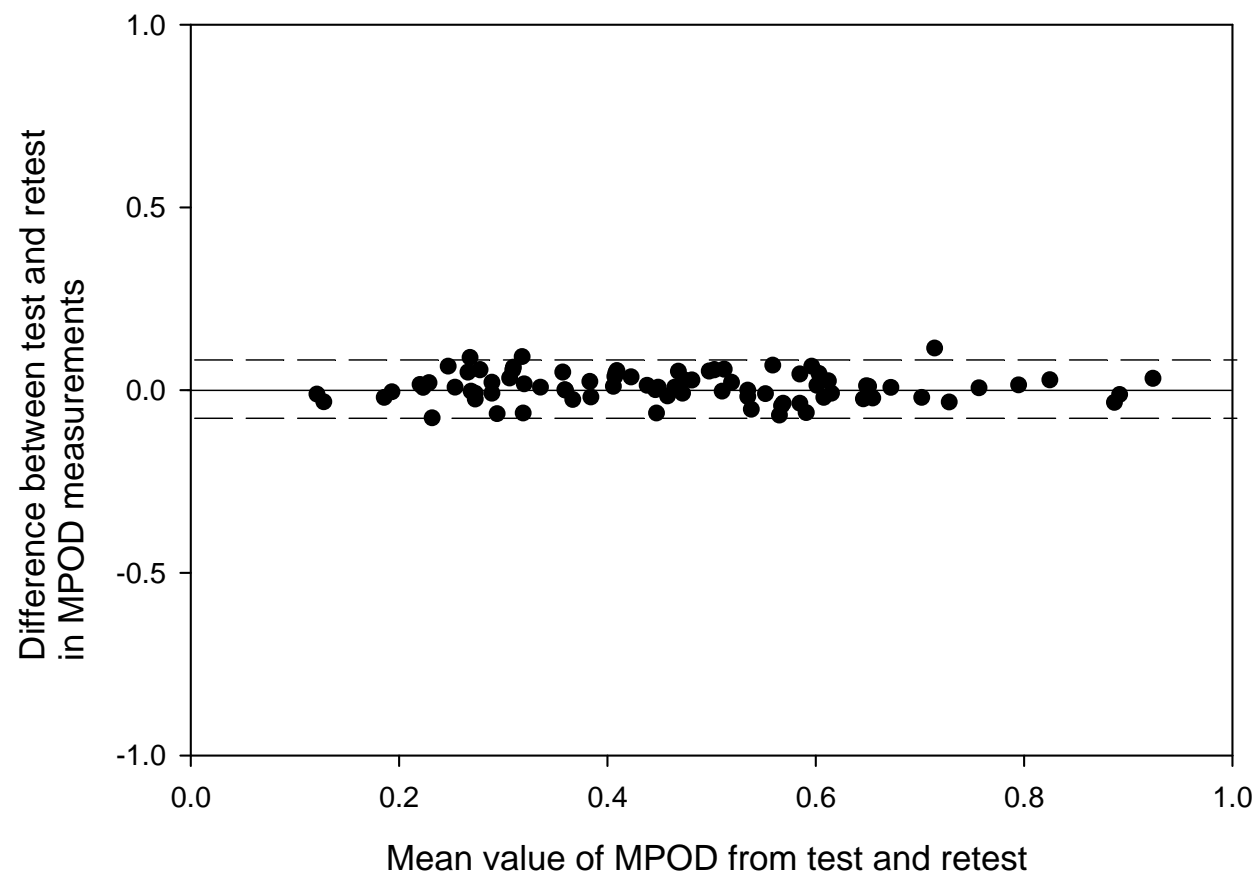

Fig. 3.1 Graph showing the Bland Altman analysis for the 83 subjects where the difference in MPODs measured during the test and retest is plotted with respect to the mean of the MPODs measured. The solid line depicts the mean of the testretest and the dashed horizontal lines are the limits of agreement.

On analysis of Fig 3.1, the average difference between the test retest of MPOD was 0.004 density units with the limits of agreement (mean \pm 2 SD) being 0.004 \pm 0.075 density units.

Results from retest of MPOD measurements were plotted with respect to the values of MPOD measured during the testing phase as shown in Fig 3.2 below. 


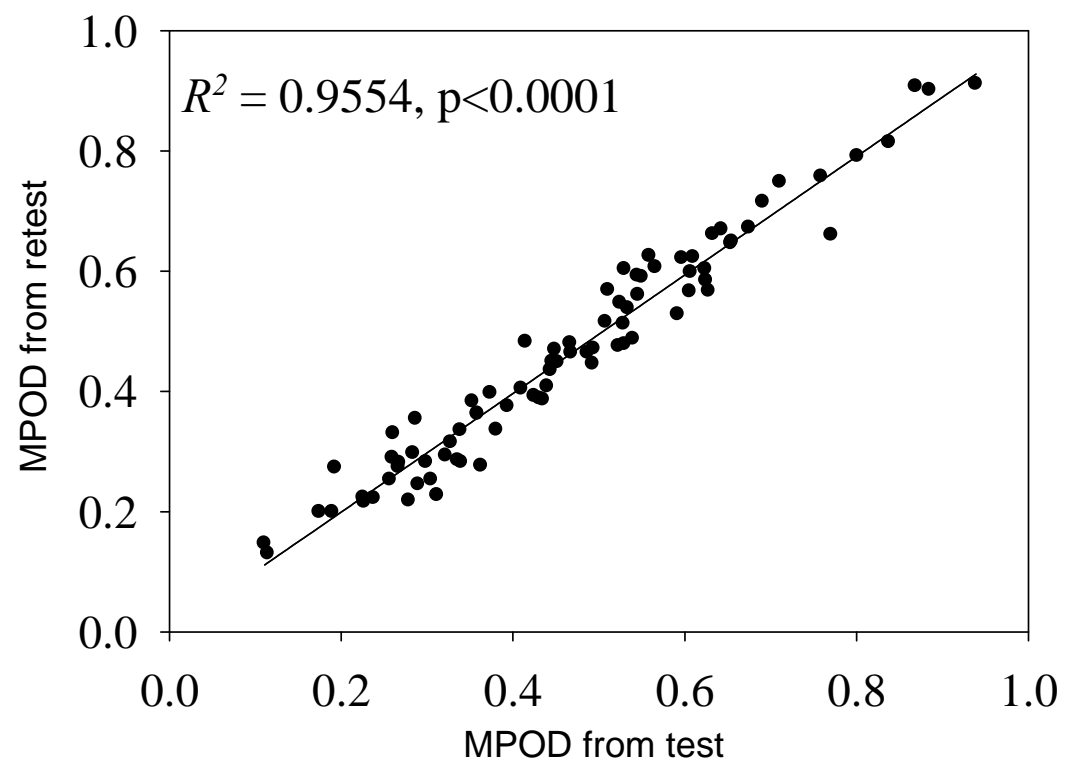

Fig 3.2 The scatter plot along with the regression analysis for MPODs measured during retest with respect to the MPODs measured during test for 83 subjects.

A regression analysis of Fig 3.2 was conducted which resulted in the $\mathrm{R}^{2}$ value of 0.9554 and p-value $<0.0001$. The Eq. of the associated regression line was $\mathrm{y}=0.9859 \mathrm{x}+2.11 \times 10^{-3}$.

As mentioned in section 2.2.2, the associated microprocessor program with Mapcatsf uses the modified age-related luminous efficiency function suggested by Sagawa and Takahashi to compute the lens optical density and lens equivalent age which in turn is further utilized to compute the MPOD values. To test the validity of the programmed model, we plotted the LEA obtained from Mapcatsf with the actual age of the subject as shown in Fig. 3.3 below. 


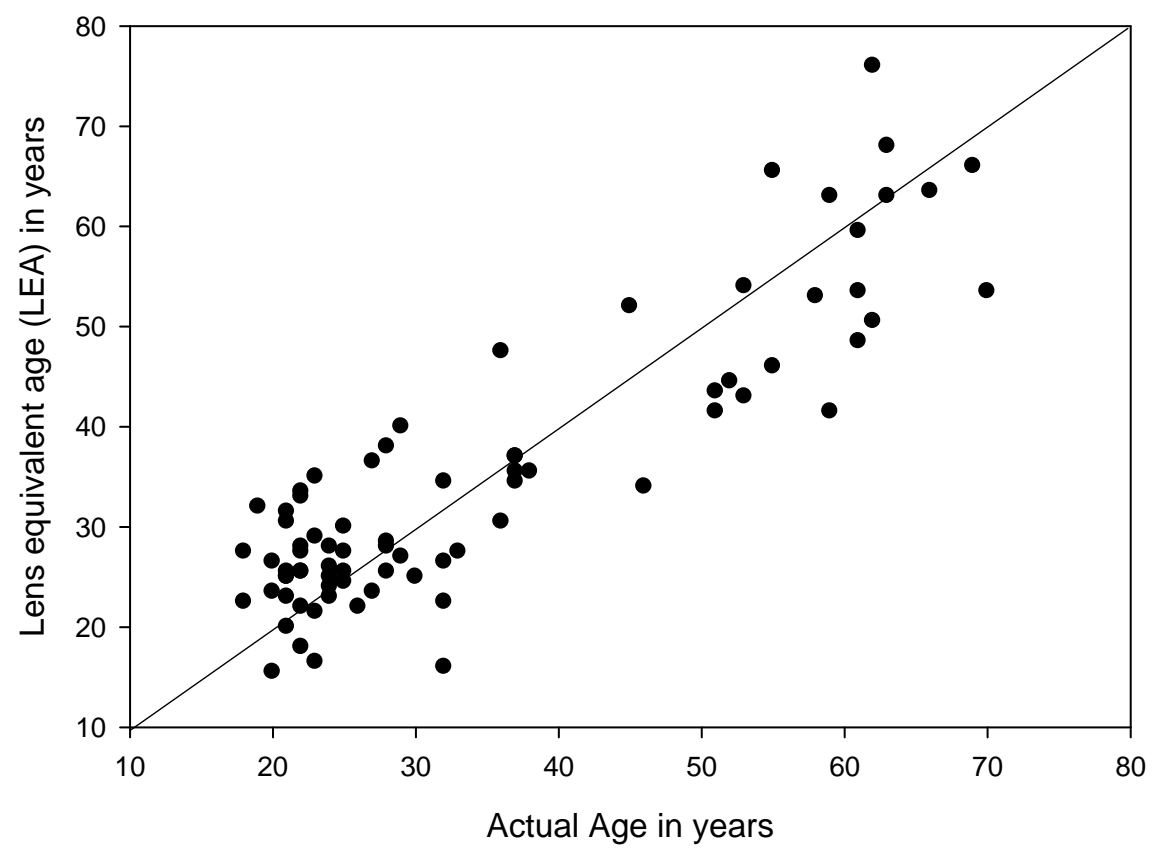

Fig. 3.3 The LEA or lens equivalent age is plotted as a function of the subject's actual age. The solid line is a line of slope unity. The points above the line are the ones for the subjects whose LEAs were more than their actual age and the points that are below are the ones for whom the LEAs were less than their actual age.

From regression analysis of the scatter plot in Fig. 3.3 the $\mathrm{R}^{2}$ value was 0.775 . The Eq. of the regression line for the plot in Fig.3.3 was of the form $y=0.807 x+6.701$.The level of agreement between the LEA and the actual age of the subjects was interpreted from the slope of the regression line which was 0.807. The high values of both the $\mathrm{R}^{2}$ and the slope of the regression line for the scatter plot in Fig. 3.3 indeed support Sagawa and Takahashi's explanation for the change in $V_{10}(\lambda)$ with age being mainly because of the changes in the optical density of the lens. In Fig. 2.6, we had observed high levels of discrepancy that arise using templates from excised lenses when compared to results from Sagawa 
and Takahashi. Since the regression analysis of Fig. 3.3 yielding high levels of agreement between LEA and actual age, using the age-dependent template developed by Sagawa and Takahashi would be the appropriate choice for LEA and MPOD measurement.

\section{Section 3.2}

\section{Testing of Mapcatsf beyond test-retest to determine its reliability}

The preliminary test-retest results with Mapcatsf have shown high levels of repeatability with a mean of the difference between test and retest of only 0.004 . The value of the difference implies that if an individual is tested over and over again, the value of MPOD would not vary significantly considering that other factors remain the same. However repeatability of measurements does not guarantee reliability in measurements obtained by an instrument. Results from further testing are presented in the following chapters to establish the reliability on the data obtained by Mapcatsf.

Because Mapcatsf is a LED-based HFP machine, either of the component LED sources might need to be replaced. From our experience, commercial LEDs purchased vary in spectrum as far as their peak wavelength is concerned by at least a few nanometers from their prescribed nominal values on their package. Ideally an instrument should not exhibit high levels of variation in measurement associated with minor variations in the spectra of the components. We investigated the effects, if any, of changing the component LEDs' peak 
wavelengths on the measured values of MPODs or LODs. The procedure was to simulate a shift in the spectra of both the component LEDs and then compare the outcomes with the results obtained for the LED pairs for which the microprocessor was originally programmed. The simulations and the details of the analysis are given in Chapter 4.

Researchers have reported age-related changes, especially a decline with age, ${ }^{57,58,59}$ in MPOD measured using instruments that employ the HFP technique but do not account for the changing levels with age of the luminous efficiency functions of the participating subjects. Change in the luminous efficiency in the short wavelength region of the spectrum with age, as attributed by Sagawa and Takahashi, ${ }^{52}$ is considered to be an artifact of the aging lens. Age-related change in the density of the lens is termed as lens-yellowing, ${ }^{46}$ which means the lens essentially absorbs more light in blue end of the spectrum for older lenses compared to the young transparent lenses. Elevation of the optical density with age will therefore contribute in higher luminance levels in the blue channel required for reporting a flicker null/minimum condition during the perifoveal test phase of measurement. We believe the result of the luminance of the blue light being high during perifoveal testing is a contributing factor to the apparent decline of the values of the MPODs in aging subjects. Therefore to confirm our hypothesis we ran simulations to check if an apparent age-related decline in MPOD is observed if the changing luminous efficiency with age is not accounted for. Details of the simulation with the analysis and results are given in Chapter 5. 
The feature of obtaining the lens density LOD, interpreted in terms of lens equivalent age or LEA, is an ancillary feature of Mapcatsf. The viewing conditions being photopic during testing by Mapcatsf, vision is mediated by the cone photoreceptors, chiefly by the $\mathrm{L}$ (long) and $\mathrm{M}$ (medium) wavelength sensitive cones. However the ratio of the L-cones to M-cones, interpreted in terms of weight, is known to have a wide range of variation in value across individuals. ${ }^{60,61}$ Such variation in weights is believed to affect lens density measurements under photopic viewing conditions. On the other hand, vision under scotopic or minimum light conditions, is rod-mediated and is associated with the rhodopsin absorbance spectrum which is known to not vary significantly across individuals. Lens densities measured under scotopic conditions, especially at absolute thresholds, are therefore considered to be more precise in value ${ }^{56,62}$ Hence an experiment was conducted to compare lens densities of subjects measured in terms of LEA under scotopic vision at absolute threshold with those measured by Mapcatsf. Details of the experiment and outcomes are described in chapter 6 .

In chapter 7 we report the capability of Mapcatsf for monitoring changing levels of MPOD. Previously in section 1.3, we have mentioned that higher levels of MPOD can lower the risk of an individual developing AMD. Studies have shown that increase in levels of concentration of MP in individuals has been possible through diet ${ }^{63}$ or supplements ${ }^{17}$ rich in the component carotenoids making up the macular pigment. Also increased levels of MPOD resulting from supplementation 
do not change significantly over time. ${ }^{17}$ Clinical practitioners along with researchers are always interested in testing new effective supplements which can enhance the concentration levels of MP in the human retina. Macular pigment optical density(MPOD) being proportional to the concentration of the pigment, and HFP being one of the preferred choices of measurement, Mapcatsf's capacity to measure changing levels of MPOD in an individual's eye was tested. Thirty three human subjects with no previously known vision problems were put on either lutein or ester derivatives of lutein, zeaxanthin and meso-zeaxanthin for six months and their MPOD levels were monitored regularly at 8 weeks interval over the supplementation period.

Carotenoids, especially lutein, have been found in traces in the ocular lens. ${ }^{64}$ Diets rich in carotenoids are known to reduce the chances of individuals developing a cataract. Near-UV and blue light exposure has been established to be one of the chief factors for an individual to develop senile a cataract. ${ }^{65}$ Taking into account the absorption spectrum of lutein, which peaks in and around $460 \mathrm{~nm}$, this carotenoid can be considered to act as a blue light filter. While it is sufficiently concentrated in the retina to potentially limit blue light damage, its concentration in the lens is too low for similar action in this organ. On the other hand, its antioxidant potential in the lens may be relevant in reducing damage to the lens and therefore reducing the risk of developing cataract. However the exact mechanism of how, or even whether, the presence of the carotenoids helps maintain an optically transparent lens is still subject for research. ${ }^{64}$ The lens derives its 
nutrients indirectly from the bloodstream, and there has been a proven relationship between levels of MP in the retina and the carotenoid level in the blood serum. Therefore analyses were conducted to see if higher levels of MPOD are associated with a more transparent lens due to the protective nature of the traces of lutein found on the lens. ${ }^{64}$ The details of the study and analytical outcomes are provided in chapter 8.

It has been debated whether MPOD measured by HFP represents the value at the edge of the image of the foveal stimulus instead of an average of the entire image area ${ }^{66,}{ }^{67}$ Bone et al. in 2004 argued against this hypothesis, attributing MPOD values that were less than the average to involuntary eye movements associated with gaze. ${ }^{48}$ In chapter 9 we present the results of an experiment that we conducted to infer if a central gaze fixating foveal stimulus can be designed which would minimize the effects of such fixational eye movements. 


\section{Chapter 4}

\section{Effects of Shifts in Spectrum of the Component LEDs}

\section{Section 4.1}

\section{Introduction}

Mapcatsf, discussed in Chapter 1, uses high power LEDs as the sources of light. The component LED sources might need to be replaced. However, commercial LEDs are available with labeled nominal values of their peak wavelengths and bandwidths, which are observed to have variability when their spectra are analyzed using a spectrophotometer.

In section 2.2, Eq.s necessary to compute the MPOD and LOD or LEA on Mapcatsf directly involve the spectral characteristics of the component LED sources. Therefore, investigation of the effects on the MPOD and LOD measurements because of variations in the matching levels of the luminance caused by shifts in peak wavelengths of the component LEDs of the stimulus, is of prime importance.

\section{Section 4.2}

\section{Theory}

The operation of the Mapcatsf for arriving at the flicker null involves adjusting the luminance of the blue LED light source while having the luminance of the green LED light source fixed. The LED sources are broad bandwidth sources. The relationship between the peak macular pigment optical density "P” and the ratio of the photodetector readings of the blue light at the foveal and perifoveal flicker 
null conditions is obtained from the polynomial fit, unique for an individual's LEA, as shown in Fig.2.4. The polynomials in Fig.2.4 are cubic in nature and are of the form as shown in Eq.1 below:

$$
P=Y_{o}+a\left(\log \left(\frac{\phi_{B F}}{\phi_{B P}}\right)\right)+b\left(\log \left(\frac{\phi_{B F}}{\phi_{B P}}\right)\right)^{2}+c\left(\log \left(\frac{\phi_{B F}}{\phi_{B P}}\right)\right)^{3}
$$

As discussed in detail in section 2.2.2, the LEA is obtained from the luminance match conditions during the perifoveal flicker null conditions in accordance with the polynomial fit to Fig. 2.5, which is of the form as shown in Eq. 2 below:

$$
L E A=Y_{o}^{\prime}+a^{\prime} \log \left(\frac{\phi_{B P}}{\phi_{G}}\right)+b^{\prime}\left(\log \left(\frac{\phi_{B P}}{\phi_{G}}\right)\right)^{2}+c^{\prime}\left(\log \left(\frac{\phi_{B P}}{\phi_{G}}\right)\right)^{3}
$$

The critical role played by the luminance match between the component LEDs is further established from the fact that values of the MPOD and the LEA measured by Mapcatsf are based on Eq.8 in section 2.2.1 and Eq.11 in section 2.2.2 respectively. Therefore, to test the effects of shifts in peak wavelengths of the LEDs, simulation experiments were conducted where individual LED spectra were shifted by the desired amounts and photodetector ratios $\frac{\phi_{B F}}{\phi_{B P}}$ and $\frac{\phi_{B P}}{\phi_{G}}$ were calculated according to eq. 8 in section 2.2.1 and eq. 11 in section 2.2.2 respectively. The generated values of $\frac{\phi_{B F}}{\phi_{B P}}, \frac{\phi_{B P}}{\phi_{G}}$ were substituted in eqns. 1 and 2 above respectively to generate the values of MPOD and LEA. The fitting coefficients used were based on the original spectra of the component LEDs. 


\section{Section 4.3}

\section{Simulations}

First we tested for the level of differences in the peak values of MPOD obtained because of shifts in the peak wavelengths of the component LEDs. We began by calculating the effect of a peak wavelength shift in the blue LED keeping the spectrum of the green LED the same. However no alteration was made to the microprocessor code to reflect such a shift. When purchasing commercial LED packs, a $\pm 5 \mathrm{~nm}$ variation in the peak wavelength is quite common. Therefore we tested the level of differences that would be observed in the MPODs for subjects with lens equivalent ages of 20, 40, 60 and 80 years when the blue LED peak wavelength was shifted by $+5 \mathrm{~nm}$ and $-5 \mathrm{~nm}$. The calculations were all made using Sigmaplot. The integrals involved in eqns. (8) in section 2.2.1 and (11) in section 2.2.2 for computing $\log \frac{\phi_{B F}}{\phi_{B P}}, \frac{\phi_{B P}}{\phi_{G}}$ (the photodetector readings for the foveal and perifoveal measurements for the simulated LED pair) were evaluated numerically using $0.5 \mathrm{~nm}$ wavelength intervals for the spectral components. The values of $\log \frac{\phi_{B F}}{\phi_{B P}}$ were substituted in Eq. 1 above to get the new peak MPODs. The effects on MPOD of the wavelength shifts are shown in Figs 4.1(a)- (d) for the different LEAs, where the percentage difference in MPOD is plotted as a function of the peak MPOD values. 


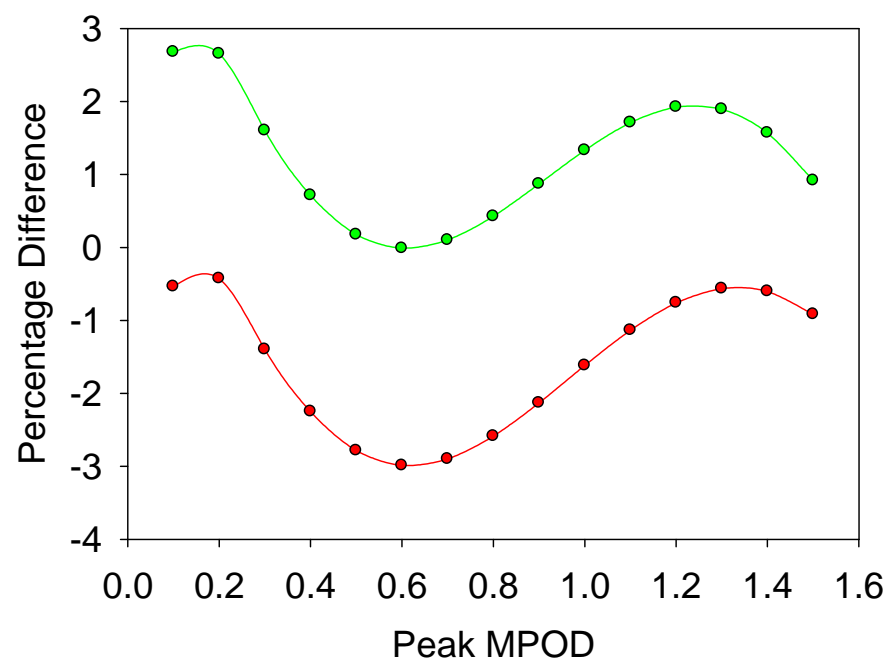

Fig.4.1a Variation in the percentage difference of MPOD observed for an individual with an LEA of 20 years when the blue LED is shifted by $5 \mathrm{~nm}$. The green line is for the variation observed when the blue LED is moved by $+5 \mathrm{~nm}$ and the red line is for the variation observed when the blue LED is moved by $5 \mathrm{~nm}$.

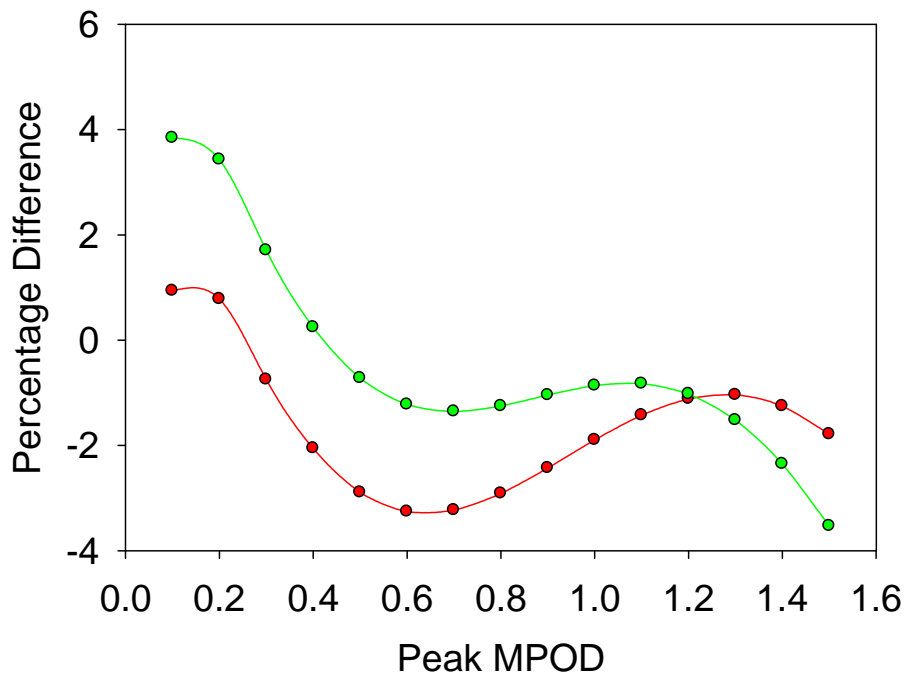

Fig. 4.1b Variation in the percentage difference of MPOD observed for an individual with an LEA of 40 years when the blue LED is shifted by $5 \mathrm{~nm}$. The green line is for the variation observed when the blue LED is moved by $+5 \mathrm{~nm}$ and the red line is for the variation observed when the blue LED is moved by $5 \mathrm{~nm}$. 


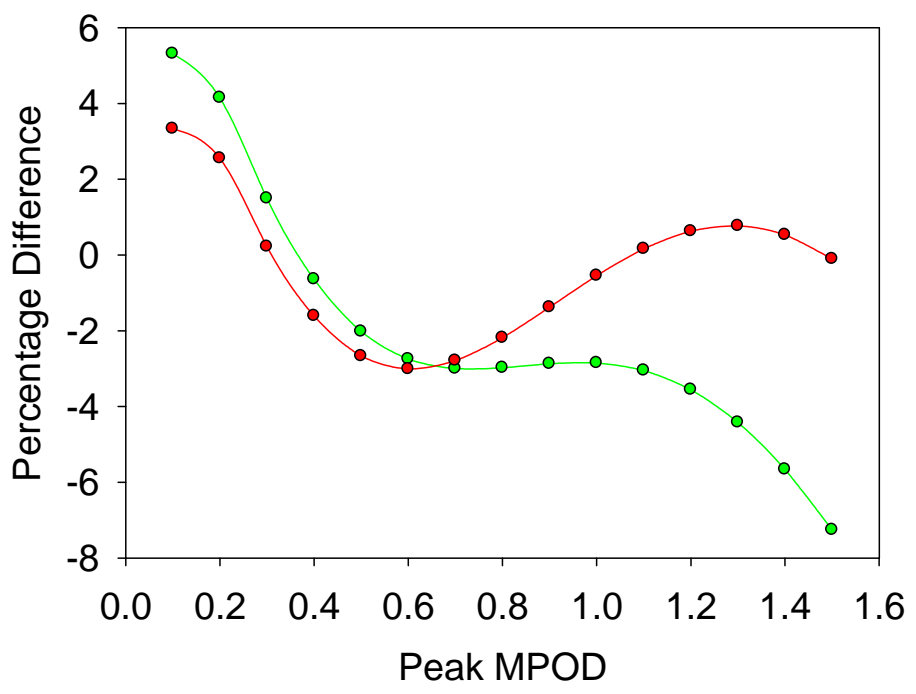

Fig. 4.1c Variation in the percentage difference of MPOD observed for an individual with an LEA of 60 years when the blue LED is shifted by $5 \mathrm{~nm}$.The green line is for the variation observed when the blue LED is moved by $+5 \mathrm{~nm}$ and the red line is for the variation observed when the blue LED is moved by $5 \mathrm{~nm}$.

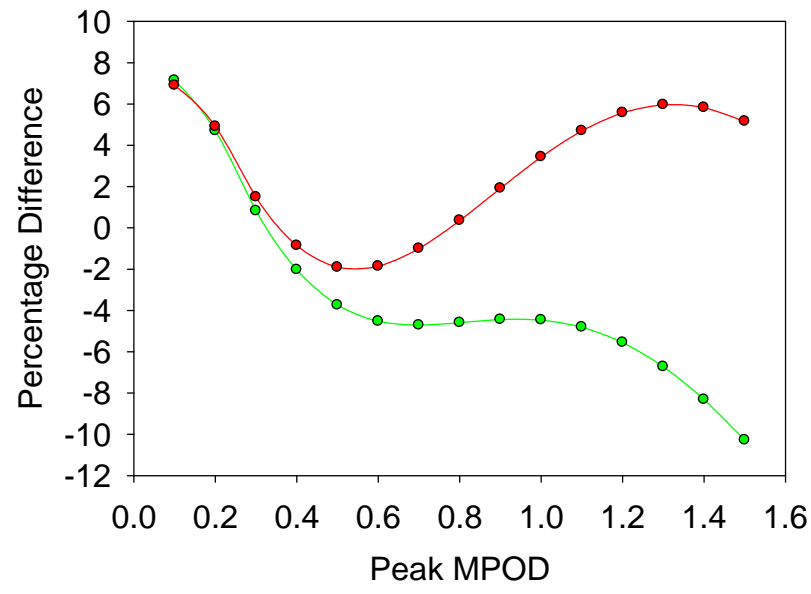

Fig 4.1d Variation in the percentage difference of MPOD observed for an individual with an LEA of 80 years when the blue LED is shifted by $5 \mathrm{~nm}$. The green line is for the variation observed when the blue LED is moved by $+5 \mathrm{~nm}$ and the red line is for the variation observed when the blue LED is moved by $5 \mathrm{~nm}$.

Effects of $\pm 5 \mathrm{~nm}$ shifts in the peak wavelength of the green LEDs were also tested while keeping the spectrum of the blue LED the same as for which the 
microprocessor was programmed. The effects of the shift in the green LEDs were tested for individuals possessing LEAs of 20, 40, 60 and 80 years just like the ones tested for shifts in the blue LED. The effects of such testing are shown in Fig.s 4.2(a)-d.

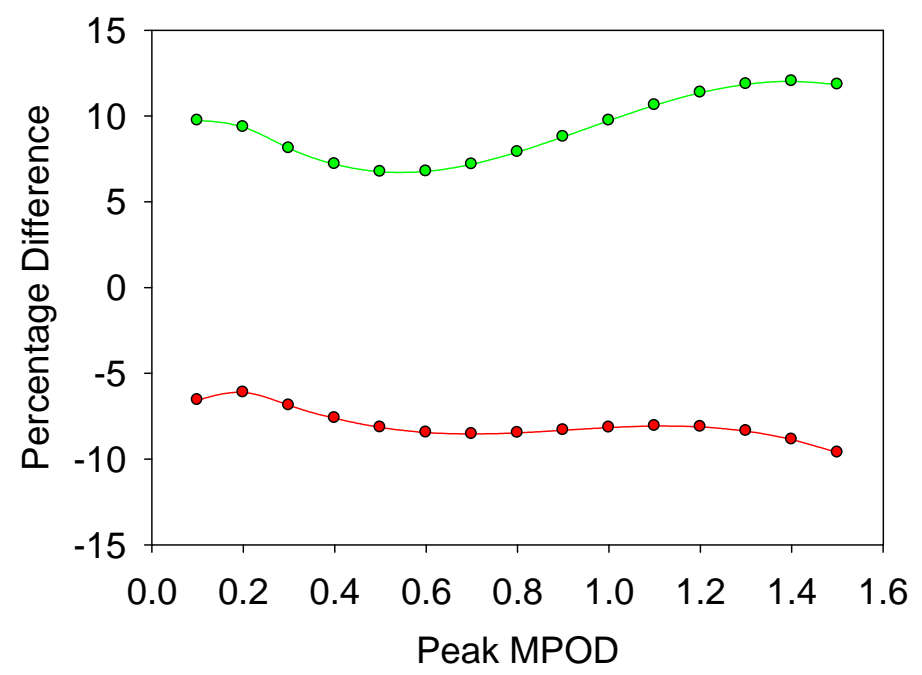

Fig 4.2a Variation in the percentage difference of MPOD observed for an individual with an LEA of 20 years when the green LED is shifted by $5 \mathrm{~nm}$. The green line is for the variation observed when the green LED is moved by $+5 \mathrm{~nm}$ and the red line is for the variation observed when the green LED is moved by $5 \mathrm{~nm}$. 


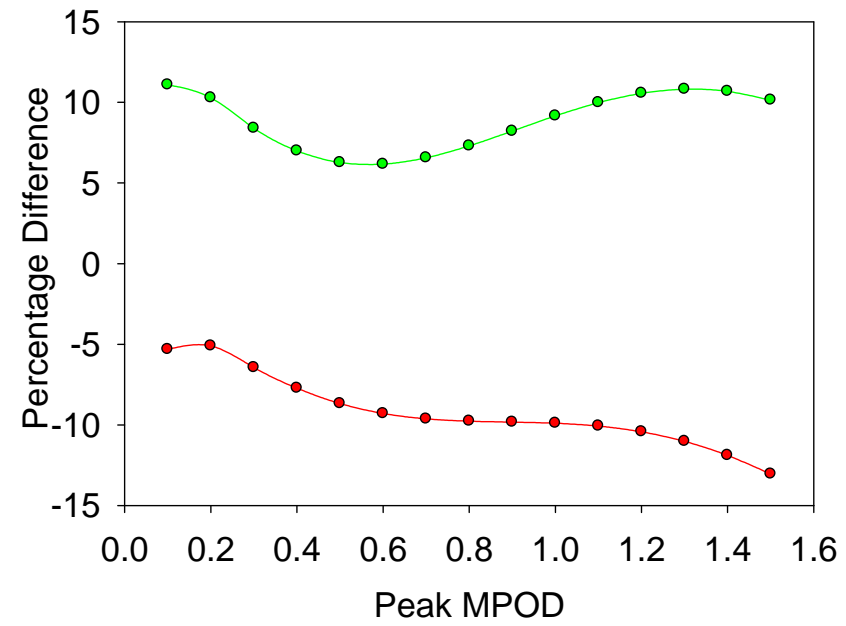

Fig 4.2b Variation in the percentage difference of MPOD observed for an individual with an LEA of 40 years when the green LED is shifted by $5 \mathrm{~nm}$. The green line is for the variation observed when the green LED is moved by $+5 \mathrm{~nm}$ and the red line is for the variation observed when the green LED is moved by $5 \mathrm{~nm}$

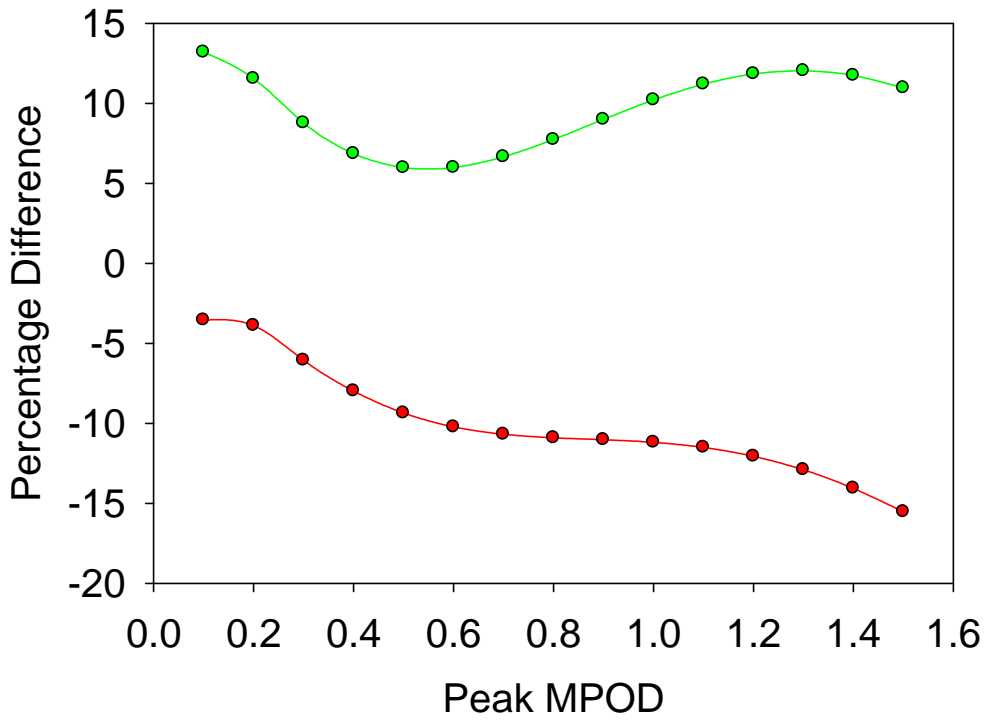

Fig 4.2 c Variation in the percentage difference of MPOD observed for an individual with an LEA of 60 years when the green LED is shifted by $5 \mathrm{~nm}$. The green line is for the variation observed when the green LED is moved by $+5 \mathrm{~nm}$ and the red line is for the variation observed when the green LED is moved by $5 \mathrm{~nm}$. 


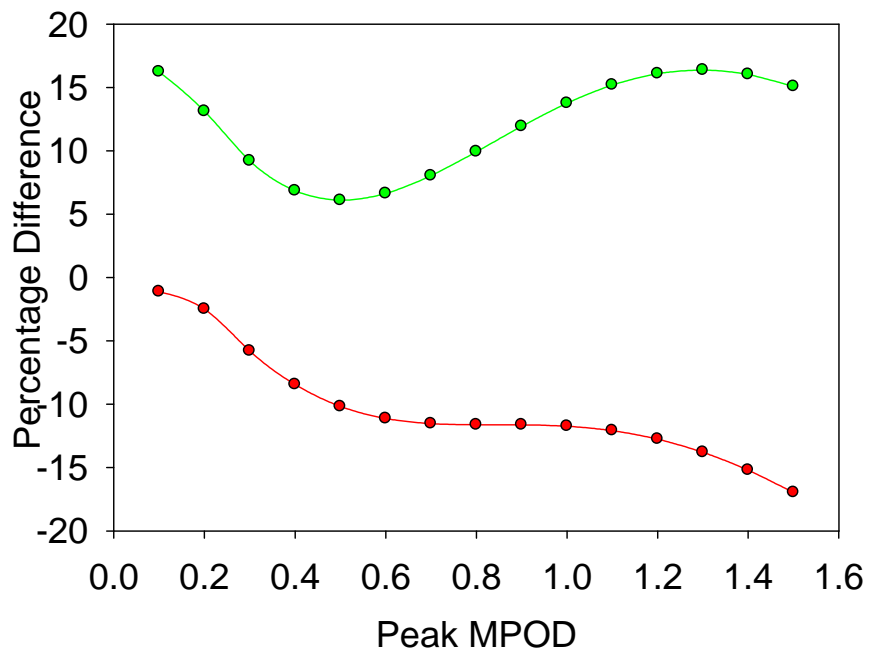

Fig $4.2 \mathrm{~d}$ Variation in the percentage difference of MPOD observed for an individual with an LEA of 80 years when the green LED is shifted by $5 \mathrm{~nm}$, The green line is for the variation observed when the green LED is moved by $+5 \mathrm{~nm}$ and the red line is for the variation observed when the green LED is moved by $5 \mathrm{~nm}$.

As mentioned in chapter2, Mapcatsf computes the lens density interpreted as lens equivalent age, LEA, from the luminance of the blue and the green LEDs at perifoveal flicker null settings according to the Eq.11 in section 2.2.2 followed by Eq. 2 in section 4.2 above. The capacity to compute the LOD and interpret it in terms of the LEA is a novel feature not available in other similar HFP instruments. Hence just like the effect of a shift in peak wavelength of the component LEDs on the measured values of the MPOD, similar testing was pursued to see the effects on the LEA. The ratio of the photodetector settings was calculated for the perifoveal flicker null conditions as mentioned above for the simulated peak shifted LED pair. The calculated values were substituted in Eq.2 of section 4.2 to obtain the LEA, but the coefficients in Eq. 2 were kept at values 
used for obtaining LEA for the original LED pair used in the instrument. At first the green LED spectrum was shifted by $\pm 5 \mathrm{~nm}$ while leaving the spectrum of the blue LED unaltered. The results of such testing are shown in Fig 4.3.

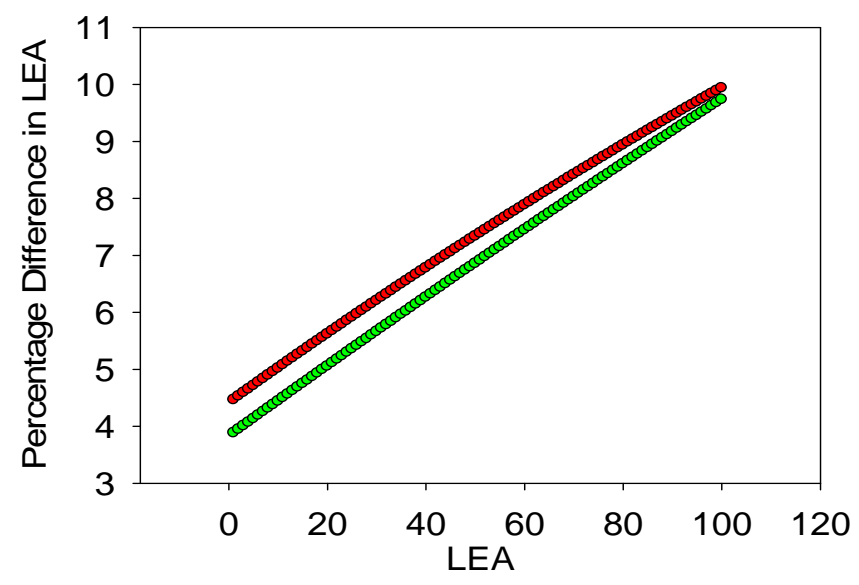

Fig 4.3 The percentage differences in LEA due to shift in the green LED by \pm 5 $\mathrm{nm}$. The green line is for the percentage differences in the LEAs that will be the result when the peak of the green LED is moved by $+5 \mathrm{~nm}$. Similarly the red line is for the percentage difference in the LEA that will result when the green LED is moved by $-5 \mathrm{~nm}$.

Further, the effect of a $5 \mathrm{~nm}$ shift in the peak wavelength of the blue LED on LEA was tested while having the spectrum of the green LED unaltered. The effect of such a shift is shown in Fig.4. Referring to Figs. 4.3 and 4.4 we can see that the shift in the blue LED would affect the LEA measurement more than an equal shift in the peak of the green LED. 


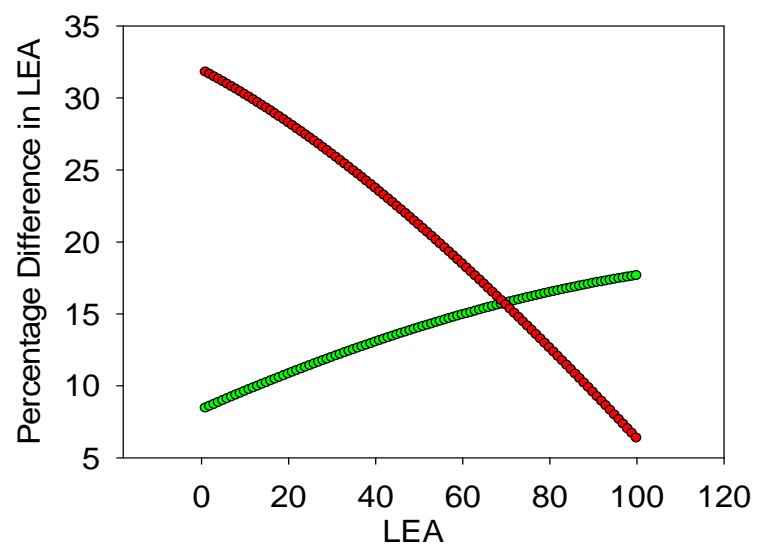

Fig.4.4 The percentage differences in LEA due to shift in the blue LED by \pm $5 \mathrm{~nm}$. The green line is for the percentage differences in the LEAs that results when the peak of the blue LED is moved by $+5 \mathrm{~nm}$. Similarly the red line is for the percentage difference in the LEA that will result when the blue LED is moved by $-5 n m$.

Five nanometer shifts being quite common in commercial LED packages, from our intuition, a 1nm shift in the peak of the LED was believed to be a more than likely occurrence. While such a shift may not be significant for other purposes, we were nonetheless prompted to test its effects on MPOD and LEA measurements based on the range of differences observed with $5 \mathrm{~nm}$ shifts in the component LEDs. The testing for the effects of $\pm 1 \mathrm{~nm}$ shifts on both the MPOD and LEA measurements were executed in the similar manner to those for the $\pm 5 \mathrm{~nm}$ shifts. The testing for the effects on the MPOD measurements was again tested for simulated individuals with LEAs of 20, 40, 60 and 80 years old. The results are shown in Figs 4.5 a-d respectively. 


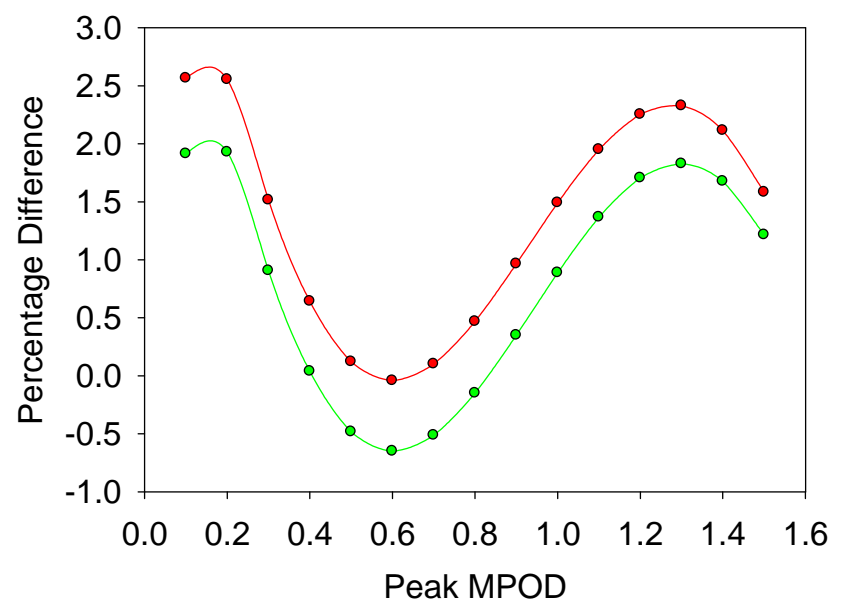

Fig. 4.5 a Variation in the percentage difference of MPOD observed for an individual with an LEA of 20 years when the blue LED is shifted by $1 \mathrm{~nm}$.The green line is for the variation observed when the blue LED is moved by $+1 \mathrm{~nm}$ and the red line is for the variation observed when the blue LED is moved by $1 \mathrm{~nm}$.

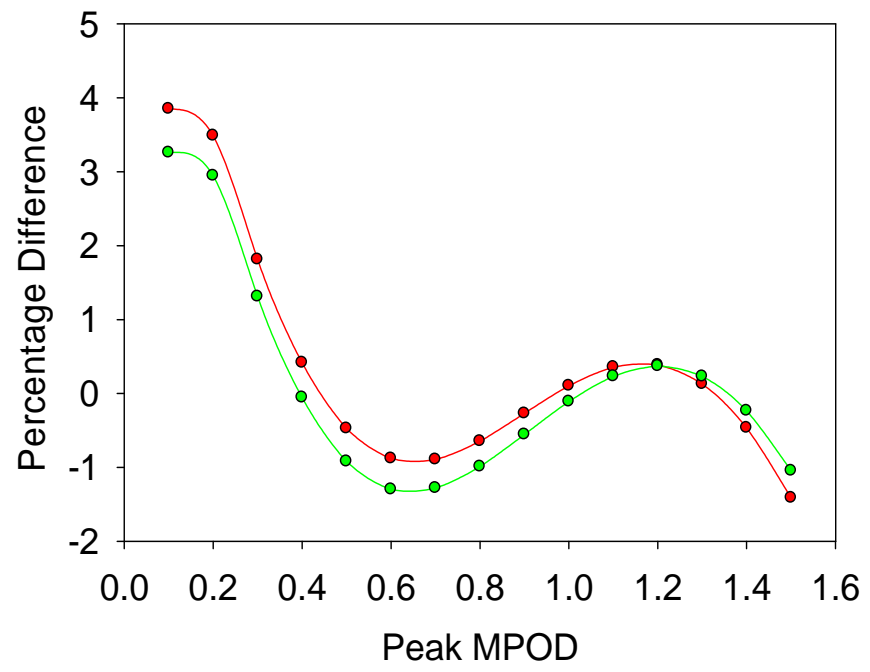

Fig. $4.5 \mathrm{~b}$ Variation in the percentage difference of MPOD observed for an individual with an LEA of 40 years when the blue LED is shifted by $1 \mathrm{~nm}$.The green line is for the variation observed when the blue LED is moved by $+1 \mathrm{~nm}$ and the red line is for the variation observed when the blue LED is moved by $1 \mathrm{~nm}$. 


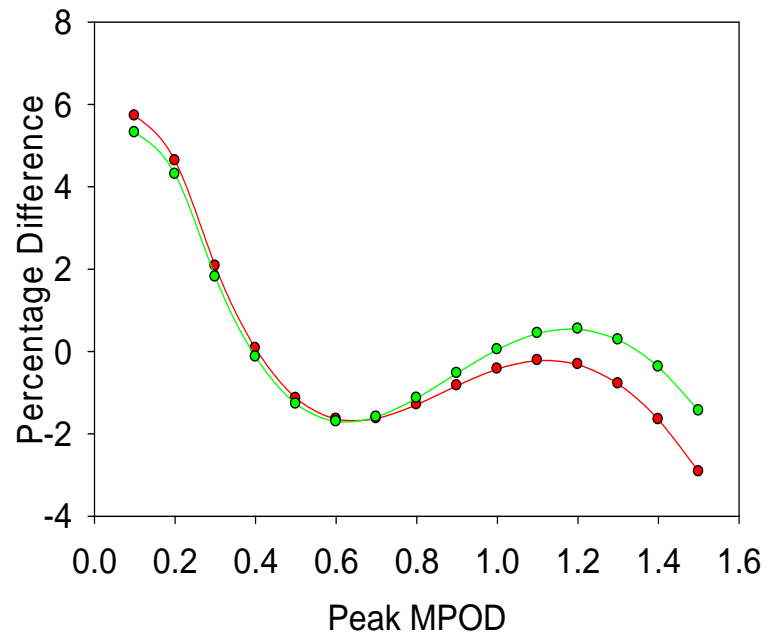

Fig 4.5 c Variation in the percentage difference of MPOD observed for an individual with an LEA of 60 years when the blue LED is shifted by $1 \mathrm{~nm}$.The green line is for the variation observed when the blue LED is moved by $+1 \mathrm{~nm}$ and the red line is for the variation observed when the blue LED is moved by $1 \mathrm{~nm}$.

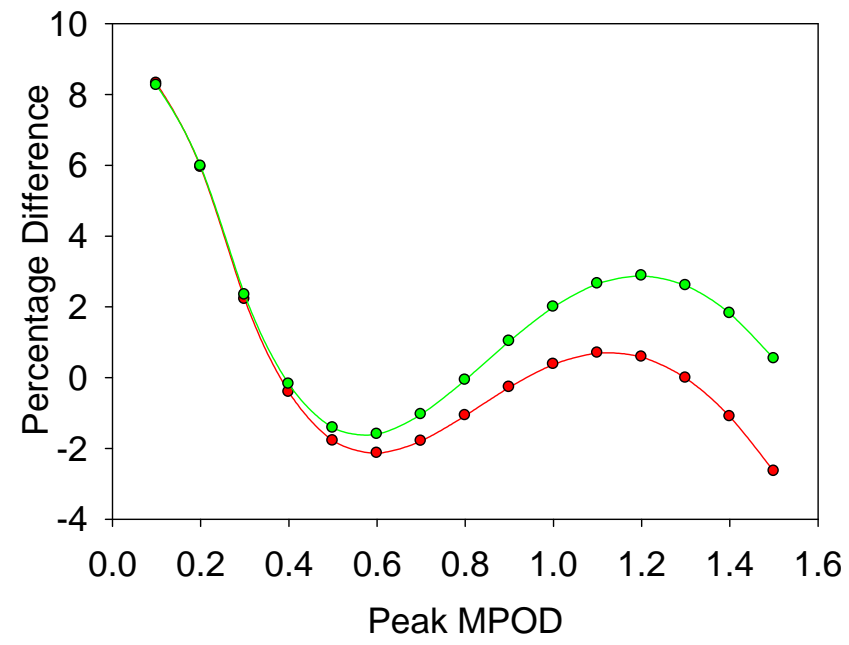

Fig $4.5 \mathrm{~d}$ Variation in the percentage difference of MPOD observed for an individual with an LEA of 80 years when the blue LED is shifted by $1 \mathrm{~nm}$.The green line is for the variation observed when the blue LED is moved by $+1 \mathrm{~nm}$ and the red line is for the variation observed when the blue LED is moved by -1 $\mathrm{nm}$. 
Similar effects resulted from $1 \mathrm{~nm}$ shifts in the green LED were investigated, and the results are shown in Fig.s 4.6 a-d.

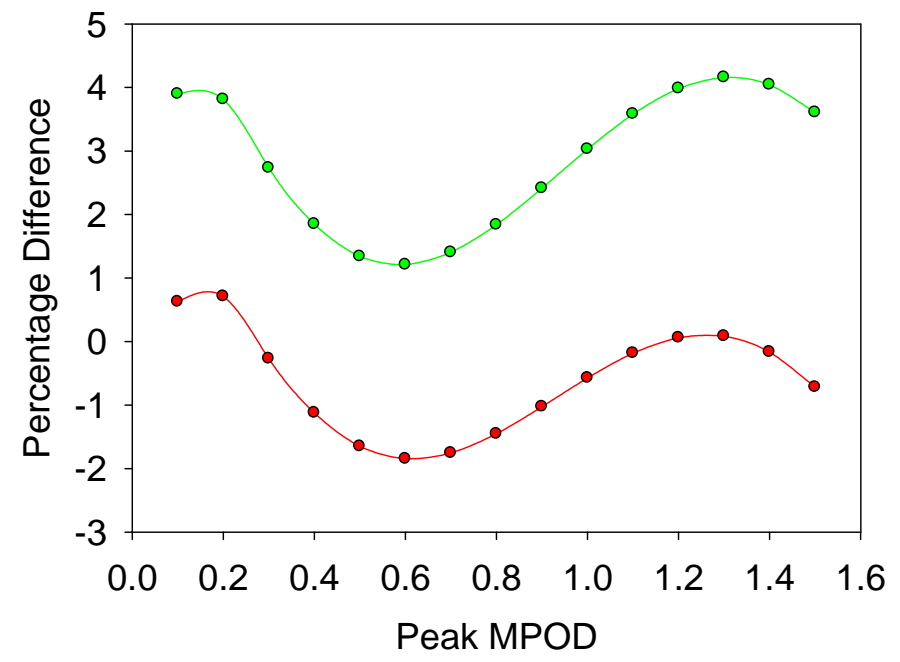

Fig 4.6 a Variation in the percentage difference of MPOD observed for an individual with an LEA of 20 years when the green LED is shifted by $1 \mathrm{~nm}$. The green line is for the variation observed when the green LED is moved by $+1 \mathrm{~nm}$ and the red line is for the variation observed when the green LED is moved by -1 nm.

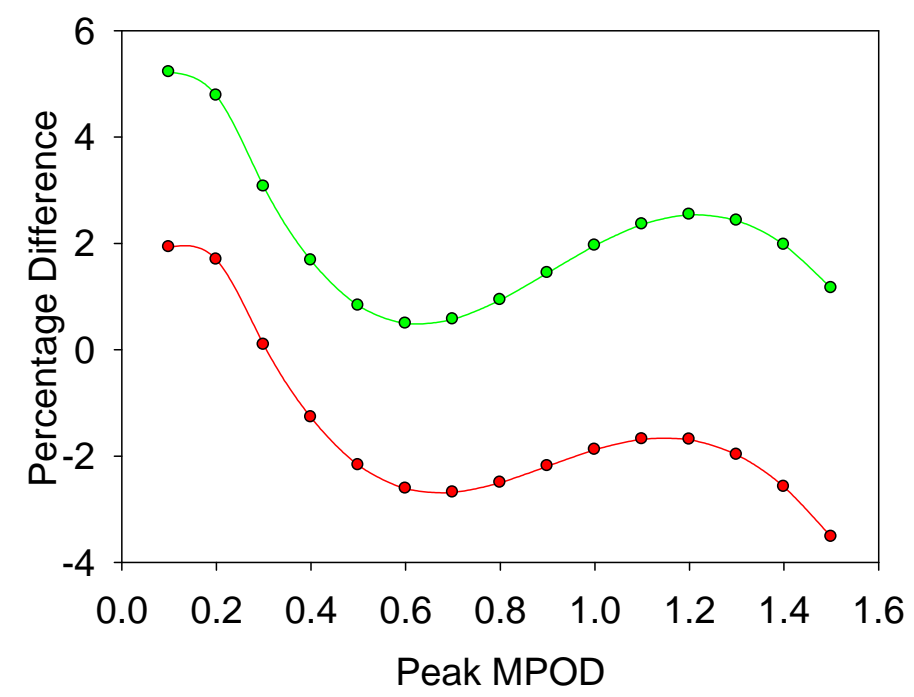

Fig $4.6 \mathrm{~b}$ Variation in the percentage difference of MPOD observed for an individual with an LEA of 40 years when the green LED is shifted by $1 \mathrm{~nm}$. The green line is for the variation observed when the green LED is moved by $+1 \mathrm{~nm}$ and the red line is for the variation observed when the green LED is moved by -1 nm. 


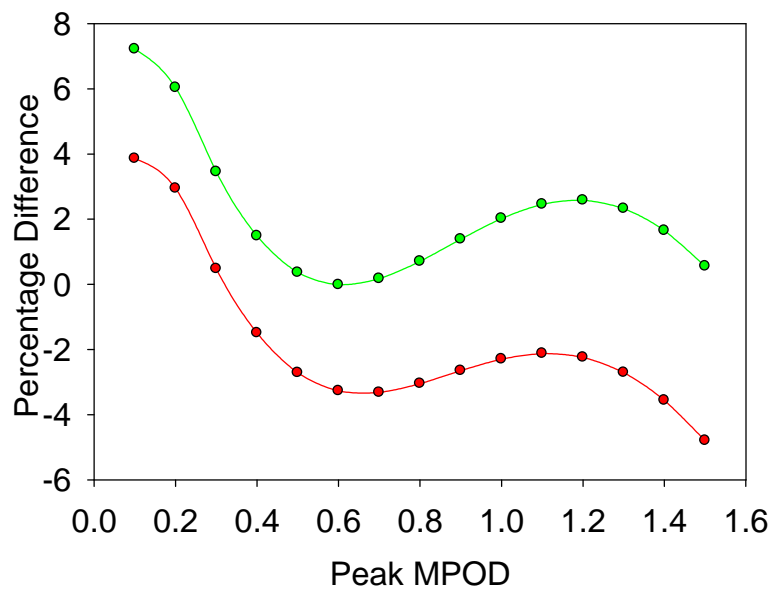

Fig 4.6c Variation in the percentage difference of MPOD observed for an individual with an LEA of 60 years when the green LED is shifted by $1 \mathrm{~nm}$. The green line is for the variation observed when the green LED is moved by $+1 \mathrm{~nm}$ and the red line is for the variation observed when the green LED is moved by -1 $\mathrm{nm}$.

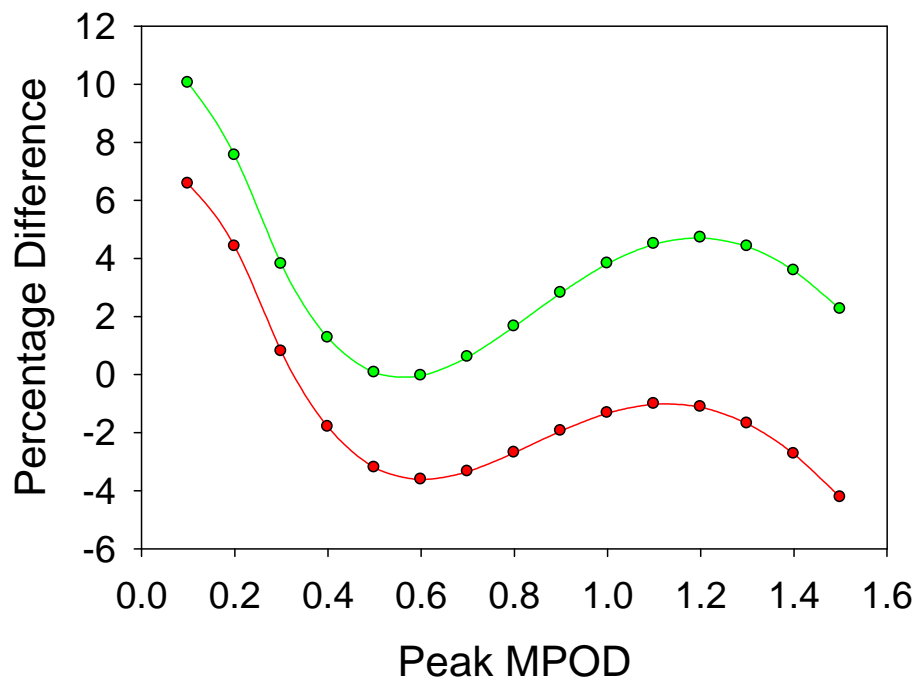

Fig4.6d Variation in the percentage difference of MPOD observed for an individual with an LEA of 80 years when the green LED is shifted by $1 \mathrm{~nm}$. The green line is for the variation observed when the green LED is moved by $+1 \mathrm{~nm}$ and the red line is for the variation observed when the green LED is moved by -1 $\mathrm{nm}$. 
Testing was also conducted for the effect of a shift in the LED peak wavelengths by $\pm 1 \mathrm{~nm}$ on the LEAs measured. The results obtained for the shifts in the blue and the green LEDs are shown in Fig.s 4.7 and 4.8 respectively.

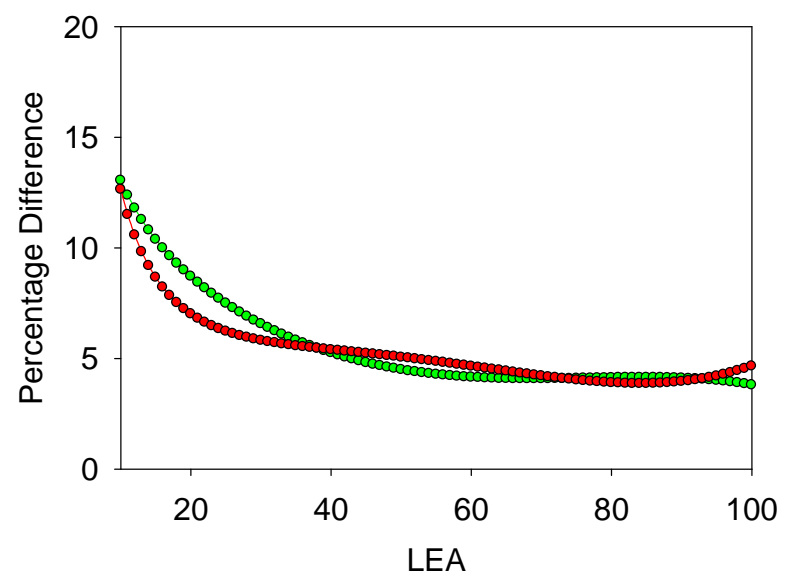

Fig 4.7 Variation in percentage difference in LEA due to shift in the blue LED by $\pm 1 \mathrm{~nm}$. The green line is for the percentage differences in the LEAs that results when the peak of the blue LED is moved by $+1 \mathrm{~nm}$. Similarly the red line is for the percentage difference in the LEA that will result when the blue LED is moved by $-1 \mathrm{~nm}$.

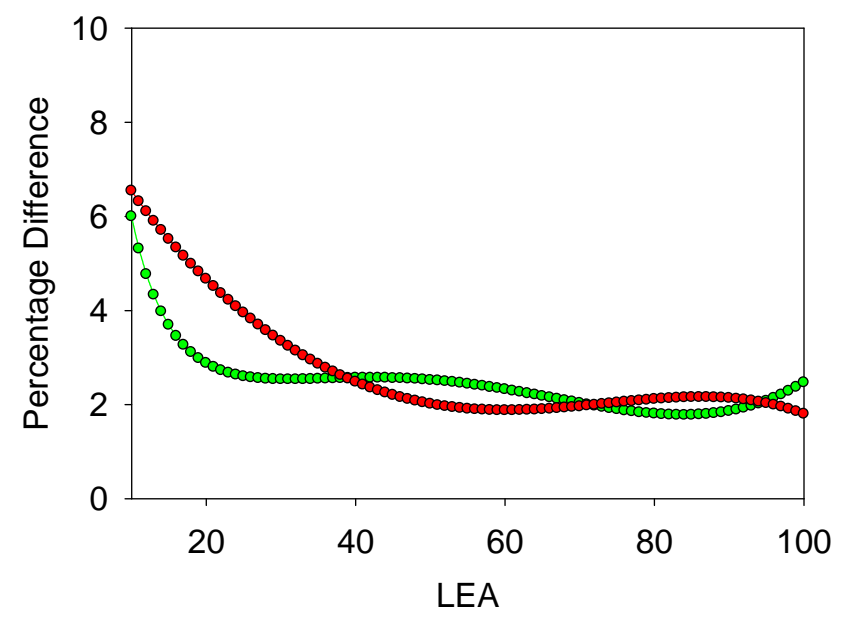

Fig 4.8 Variation in percentage difference in LEA due to shift in the green LED by $\pm 1 \mathrm{~nm}$. The green line is for the percentage differences in the LEAs that results when the peak of the green LED is moved by+1nm. Similarly the red line is for the percentage difference in the LEA that will result when the green LED is moved by $-1 \mathrm{~nm}$. 


\section{Section 4.4}

\section{Discussion}

The results of the simulated experiments conducted by shifting the LED peaks indicate significant effects on both the MPOD and the LEA measurements. The effects on MPOD measurements due to the shifts in either of the component LED spectra are seen to be dependent on the age of the subject or more precisely his/her LEA. At first we tested the effects of $\pm 5 \mathrm{~nm}$ shifts of the component LED spectra. The effects for the $\pm 5 \mathrm{~nm}$ shifts in the green LED spectrum while having the blue LED spectra fixed, on MPOD measurements were shown in Figs 2.1 a- d. Similarly effects on MPOD measurements because of $\pm 5 \mathrm{~nm}$ shifts in the blue LED spectra, but having the green LED spectra fixed were shown in Figs. 2.2 a-d. On inspection of the two sets of graphs we can observe that the overall effects are more pronounced with the increase in the associated LEAs of the subjects. Aside from an age-related effect, shifts in the green LED seem to be affecting each age group more than similar such shifts for the blue LED. Capacity to measure the lens density being a novel, ancillary feature of Mapcatsf, we tested the effects of $\pm 5 \mathrm{~nm}$ shifts in the peak wavelength for both the green and the blue LEDs on the LEA as percentage differences. Such simulated testing evolved a reverse trend, based on the effects observed in Fig.s 4.3 and 4.4 where we observe that shifts in the blue LED source affected the values of LEAs more than similar such shifts in the green LEDs, contrary to the effects of the $\pm 5 \mathrm{~nm}$ shifts on MPOD measurements. 
As mentioned in the earlier sections of this chapter, $\pm 1 \mathrm{~nm}$ shifts were assumed to be a more likely occurrence in a commercial LED pack than $\pm 5 \mathrm{~nm}$ variations. From similar such tests as those conducted for the $\pm 5 \mathrm{~nm}$ shifts, the results of the $\pm 1 \mathrm{~nm}$ shifts of both the component LEDs on the MPOD and LEA measurements are shown in Figs 4.5(a)-(d), 4.6 (a)-(d), 4.7, 4.8 . The effects are shown as percentage differences in MPODs that are outcomes of the $\pm 1 \mathrm{~nm}$ shifts in the peak wavelength of the blue LED in Figs. 4.5 (a)-(d). Similarly effects due to \pm 1 nm shift in the green LED are shown in Figs. 4.6(a)-(d). Figs. 4.7 and 4.8 depict the effects on LEA measurements due to shifts of magnitude $\pm 1 \mathrm{~nm}$ in the blue and the green LED spectra respectively.

On inspection of the Figs. 4.5 (a)-(d) and Figs. 4.6 (a)-(d) we can infer an increase with LEA in erroneous outcomes due to shift in the component LED spectra on MPOD measurements. As expected, the percentage differences in the MPOD values observed are less compared with those observed for the $\pm 5 \mathrm{~nm}$ shifts at the same LEA. Similar to the $5 \mathrm{~nm}$ shifts, a $1 \mathrm{~nm}$ shift in the green LED has more effect on MPOD measurement compared to a similar such shift of the blue LED, for the same LEA. However, similar to the $5 \mathrm{~nm}$ shifts, the percentage differences in LEA measurements are seen to be more affected with the shift in the blue LED than with shifts of similar magnitude with the green LED.

Trends observed in the plots depicting the effects on MPOD measurement due to both the $\pm 5 \mathrm{~nm}$ and $\pm 1 \mathrm{~nm}$ shifts in peak wavelengths of each of the component 
LED spectrum makes it inconclusive to comment which direction of shift in the spectra has a more pronounced overall effect than the other.

In Fig 4.3 one can observe that the effect of a $+5 \mathrm{~nm}$ shift in the green LED peak has a lower effect than a $-5 \mathrm{~nm}$ shift on the LEA measurements. However in case of Fig. 4.8 the effects of $\pm 1 \mathrm{~nm}$ shifts in the green LED on LEA measurements cannot lead to an inference as to shifts in which direction of the spectrum of the green LED might lead to less margin of error.

On observing Fig 4.4, we can again comment that a $+5 \mathrm{~nm}$ shift in the blue LED has less effect than a $-5 \mathrm{~nm}$ shift on LEA measurements. The effects of shifts in the blue LED spectrum by $\pm 1 \mathrm{~nm}$ shown in Fig. 4.8 again cannot aid in making a conclusive comment whether a positive or a negative shift in the peak of the blue LED by $1 \mathrm{~nm}$ might have more effect on the LEAs measured.

In conclusion, since we have observed a significant variation in both the MPOD and the LEA due to shifts in the component LEDs by even 1nm, if the LEDs need to be replaced, care should be taken that their spectrum is not shifted even by 1 $\mathrm{nm}$ from that of either of the component LEDs for which the microprocessor was originally programmed. Further, in our opinion, it will actually be better to reprogram the microprocessor if the LEDs are replaced, taking into account their precisely measured spectra so that erroneous values of MPOD and LEA are not obtained. 


\section{Chapter 5}

\section{Variation of Peak Macular Pigment Optical Density with Age measured using Heterochromatic Flicker Photometry}

\section{Section 5.1}

\section{Introduction}

Researchers over the years have reported various trends of an overall variation of MPOD with age of the subjects. Some have reported an overall rise, ${ }^{68,69,70}$ some an overall decline $e^{57,67}$, or some no change at all ${ }^{33,71,72}$. However, especially for the investigators employing HFP, a majority of them have reported an overall negative relationship between age and $\operatorname{MPOD}^{58,57,32,73}$. After an investigation of the various instruments used when MPOD was measured by HFP, we observed that the developers of these instruments ${ }^{74,41}$ had not taken into account the changes in the luminous efficiency of an individual with age. The change in the luminous efficiency with age is primarily observed in the lower wavelength region of the visible spectrum; ${ }^{52}$ such a change therefore can be attributed mainly to the change in the optical density of the lens with age. ${ }^{62,52,46}$ We conducted an experiment in order to test if an apparent age-related decline in MPOD, similar to those reported by other researchers using HFP, can be explained as a result of overlooking the age-related variation in luminous efficiency function in the instruments used. 
Sets of unbiased random numbers were used to simulate subjects' MPOD and LEA. The simulated data were used to compute the photodetector ratio $\frac{\phi_{B F}}{\phi_{B P}}$ which was then used in eq.1 in section 4.2, but with the fitting coefficients kept fixed for those of an individual aged 32 years, to compute the value of "partially corrected” MPOD.

\section{Section 5.2}

\section{Theory}

Fig. 5.1 below shows the age-corrected luminous efficiency function $V_{10}(\lambda)$, for a centrally viewed 10-degree stimulus, corrected for age using the template suggested by Sagawa and Takahashi. The functions $V_{10}(\lambda)$ when age-corrected are termed $V_{10}(\lambda, a)$, with the parameter " $a$ ” depicting the LEA as explained in detail in chapter 2. On inspection of these functions, more pronounced age-related changes are observed in the lower wavelength region (400 to $550 \mathrm{~nm}$ ) than in the higher wavelength region. 


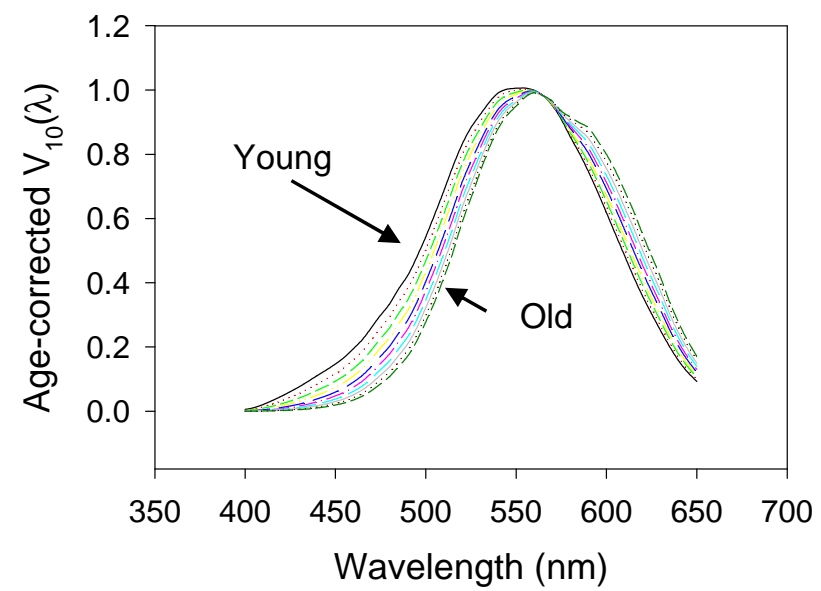

Fig 5.1 The age corrected luminous efficiency function for a centrally viewed 10 degree stimulus.

On inspection of the Eq.s given in chapter 2 used for computing the ratio between the photodetector outputs, which are subsequently used to compute the MPOD values, we can clearly observe that the age-corrected luminous efficiency function plays an integral role. 


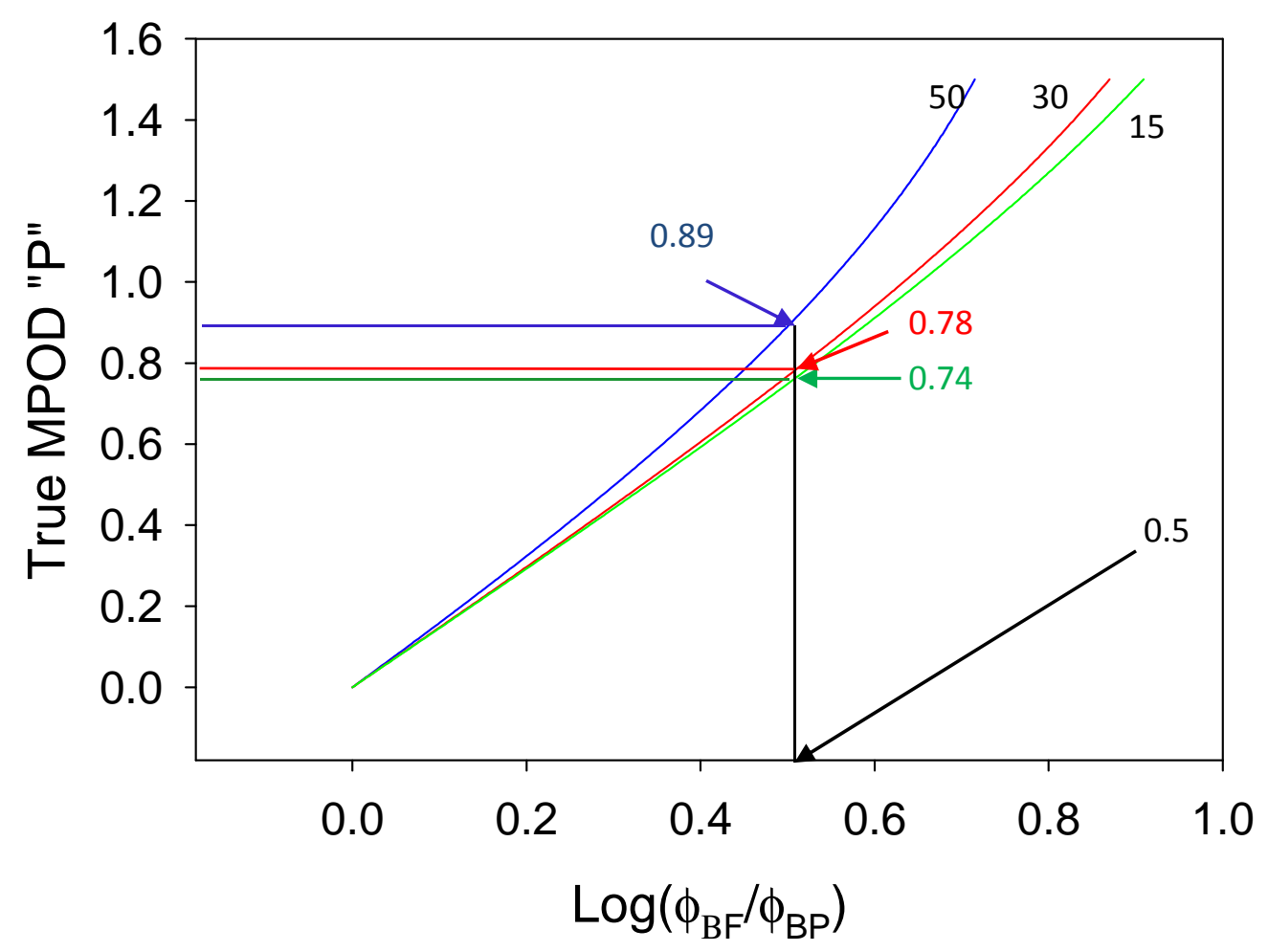

Fig.5.2 Plot of peak MPOD “ $P$ ” with $\log \left(\frac{\phi_{B F}}{\phi_{B P}}\right)$ for 50, 30 and 15 year olds.

The effect of having the fitting coefficients in the polynomial not corrected for LEA is illustrated in Fig. 5.2 above. Here it can be seen that if an individual of 30 years of age gave a $\log \left(\frac{\phi_{B F}}{\phi_{B P}}\right)$ value of 0.5 , following the curve for the associated LEA of that of 30 years, the MPOD would be computed as 0.78 by the microprocessor. If a subject with an LEA of 50 years gave the same $\log \left(\frac{\phi_{B F}}{\phi_{B P}}\right)$ of 
0.5 , the peak MPOD would be computed as 0.89 , and someone with an LEA of 15 years would give a true MPOD of 0.74 for the same $\log \left(\frac{\phi_{B F}}{\phi_{B P}}\right)$ value of 0.5 .

\section{Section 5.3}

\section{Experiment}

We generated unbiased random numbers using Sigmaplot ${ }^{\circledR}$ to simulate peak MPOD values between 0 and 1, and LEA values between 20 and 90 years. See Fig. 5.3. The values of peak MPOD show no dependence, on average, with age as is evident from the slope of the regression line which is $5.736 \times 10^{-5}$ density units/year.

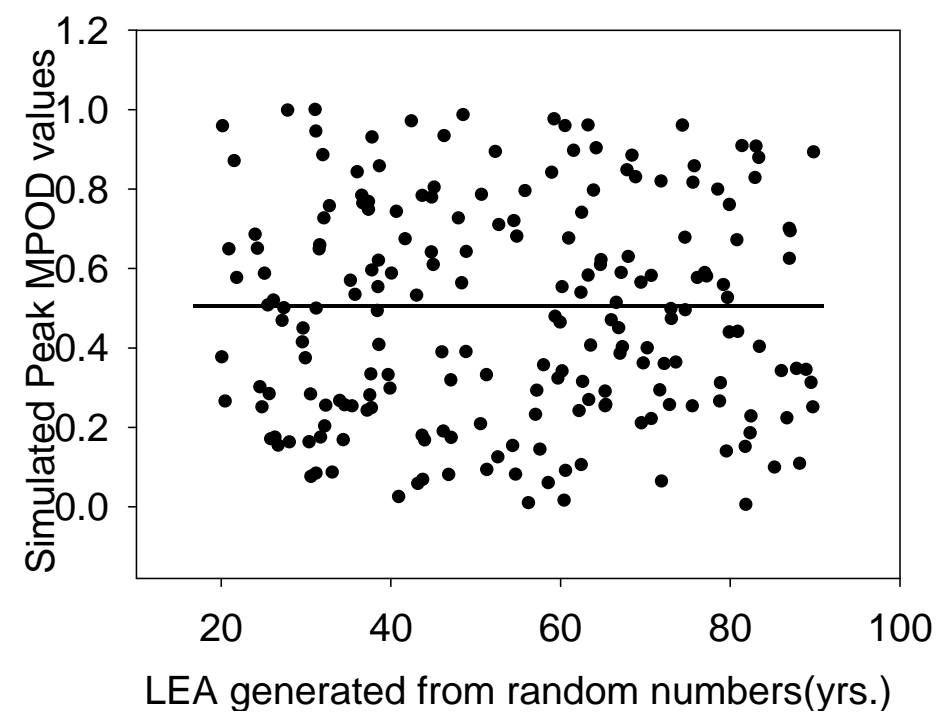

Fig 5.3 The scatter plot of simulated peak MPOD values with the LEAs generated from random numbers. 
Employing eq. 8 in section 2.2.1, the $\log \left(\frac{\phi_{B F}}{\phi_{B P}}\right)$ values were calculated for each individual on Sigmaplot. The calculated values of $\log \left(\frac{\phi_{B F}}{\phi_{B P}}\right)$ are plotted with respect to the LEAs in Fig 5.4. The peak MPOD obtained by Mapcatsf is computed from $\log \left(\frac{\phi_{B F}}{\phi_{B P}}\right)$ using eq. 1 in section 4.2. The coefficients in the Eq. are dependent on the LEAs of the individuals obtained from eq. 2 in section 4.2. Therefore, to test the effect of the invariance in the luminous efficiency function, we kept the values of the coefficients, $Y_{0}, a, b, c$ for those of a 32 year old individual, hence giving "partially corrected” values of MPOD. Such an age was selected because the standard luminous efficiency curve approved by the CIE is based on the data gathered from a group of subjects where the average age was 32 years. ${ }^{75}$

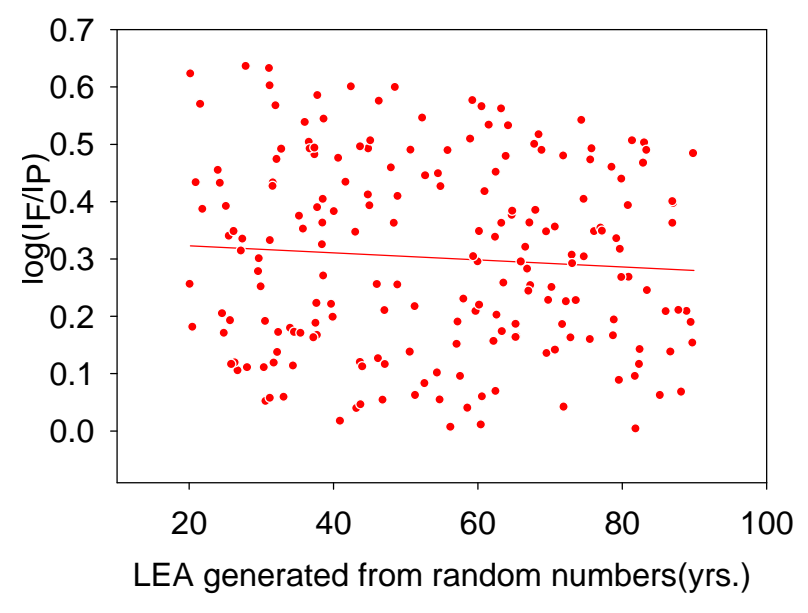

Fig 5.4 Plot of $\log \left(\frac{\phi_{B F}}{\phi_{B P}}\right)$ values versus LEAs generated from random numbers 
Six independent data sets were generated similar to that described by Fig. 5.3 and the simulated values of $\log \left(\frac{\phi_{B F}}{\phi_{B P}}\right)$ were calculated in the same manner as described above. Following this, the calculated values were partially corrected as mentioned above to obtain the MPOD values if the age correction for the luminous efficiency function is not incorporated. An example of a test run is given in Fig.5.5.

The regression analysis conducted on the plots of MPOD vs generated LEA for 6 simulated datasets gave an average decline in MPOD of $(1.44 \pm 0.51) \times 10^{-2}$ per decade.

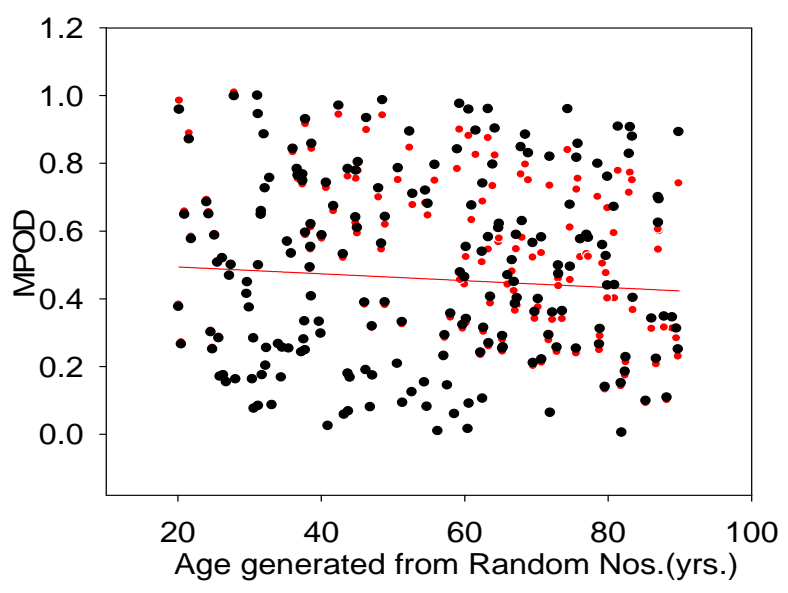

Fig 5.5 Scatter Plot of MPOD with age generated from random Nos. The red dots or the "partially corrected" MPOD values are obtained when the values of are $\log \left(\frac{\phi_{B F}}{\phi_{B P}}\right)$ used for the polynomial fit with coefficients fixed for that of a 32

year old in eq.1 in section 4.2. The red line gives the regression analysis of the variation of MPOD with age. The black dots depict the true simulated MPODs values of the subjects generated from the random numbers on Sigmaplot.

We can observe in Fig.5.5 that the "partially corrected" MPOD values for the simulated subjects in the higher age range (> 32 years) have lower calculated 
values of peak MPOD than their actual values represented by the black dots, and the partially corrected MPOD values for the subjects in the lower age group $(<32$ years) have higher values compared to their actual peak values of their MPODs. Such a trend can be explained based on the assumed invariance in the luminous efficiency function with age.

\section{Section 5.4}

\section{Discussion and Conclusion}

The average decline in peak MPOD with age per decade as observed from the average of the slopes of the six simulations was of the same order of magnitude as those reported by other researchers who had analyzed the MPOD measured by heterochromatic flicker photometry (HFP) as a function of subject age. $57,58,59,73$ Hence we can surmise that MPODs measured using broad-band light sources, especially LEDs, can give potentially erroneous results if the changing levels of the luminous efficiency function with age are not taken into account. The resultant discrepancies are more pronounced in the higher age groups than the ones observed in the younger age groups.

Mapcatsf takes into account the age-related changes in the luminous efficiency function. Hence for the same population of subjects tested for repeatability in Chapter 3, data was analyzed by plotting a scatter plot of the average MPOD obtained from test and retest versus the lens equivalent age as shown in Fig. 5.6. 


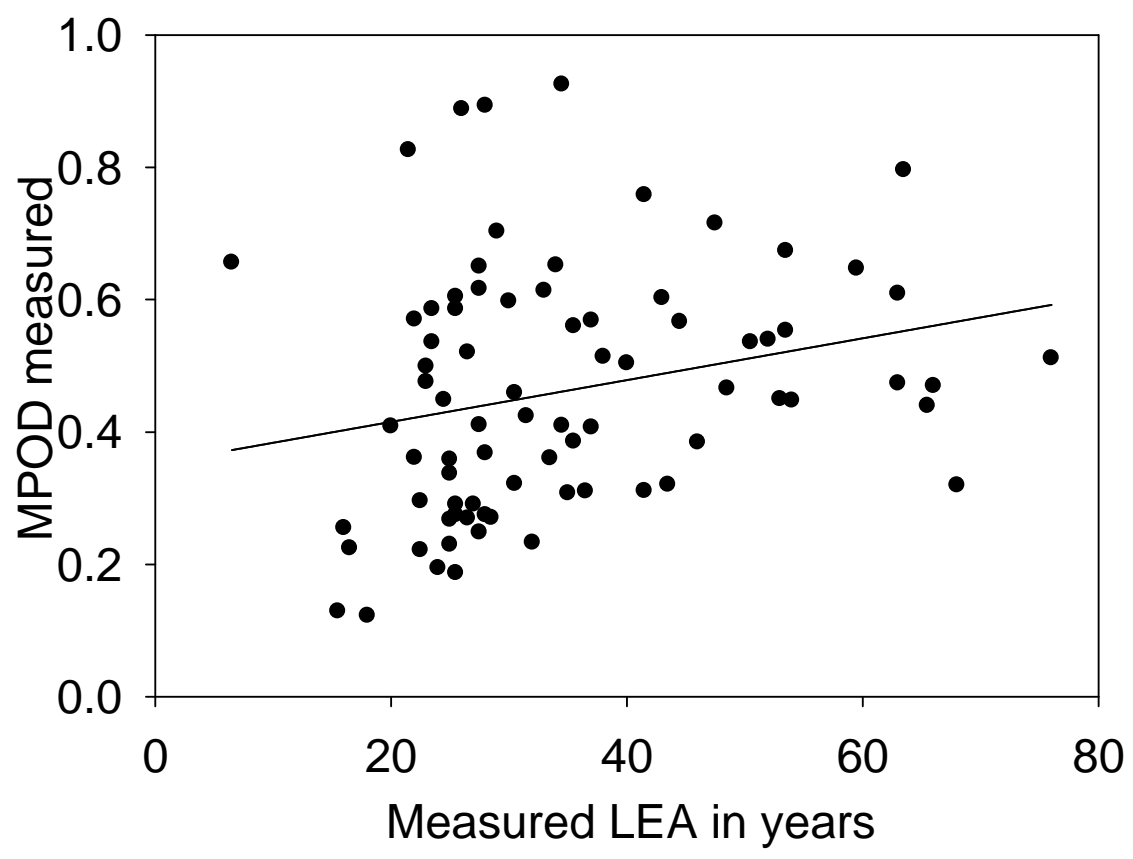

Fig.5.6 The plot of MPOD measured versus the LEAs measured while testing for repeatability of Mapcatsf. The regression line has Eq. of the $y=0.352+3.155 \times 10^{-}$ ${ }^{3} \mathrm{x}$.

The slope of the regression line in Fig 5.6 gave an overall rise of MPOD of $3.15 \times 10^{-2}$ per- decade with a p-value of less than 0.03 and $\mathrm{r}^{2}$ value of 0.061 . Recruitment of subjects was not controlled, based on lifestyle, especially diet. However, it might be possible that individuals in the higher age groups were consuming a more healthy diet rich in carotenoids, especially lutein and zeaxanthin. This possibility might be responsible for the observed rise in MPOD with age. Nevertheless, based on the statistics and the recruitment profile, a firm conclusion cannot be inferred. 
The negative trends in the variation of MPOD with age that we observed for the simulated data sets along with the positive slope of the regression line for Fig 5.6, does not lead us to a firm conclusion about the variation of MPOD with age. Researchers should therefore be cautious about the various factors such as dietary habits and levels of UV exposure of the subjects involved before predicting an age-related trend in the level of optical density of MP, particularly if it is measured by LED-based HFP instruments. 


\section{Chapter 6}

\section{Validity of Mapcatsf's capacity to measure lens optical density}

\section{Section 6.1}

\section{Importance of developing reliable lens density measuring techniques}

Cataract, manifested by the ocular lens becoming heavily opaque, ${ }^{76}$ is one of the major causes of visual impairment in the world. ${ }^{77}$ The primary structures of the lens which undergo changes in the optical density and potentially exhibit the condition of cataract are the cortex, nucleus and posterior sub-capsular (PSC) regions of the lens. ${ }^{78,79}$ Different reasons account for the accelerated increase in the optical density of these three regions of the lens. The component protein molecules undergoing changes in shape is primarily associated with the changes in the optical density of the nucleus, whereas the stress in the associated lens fibers is responsible for the rise in optical density levels of the cortical region. ${ }^{76}$ However opacity levels in the PSC region are considered mainly to be associated with diabetes, myopia, exposure to ionizing radiation and intake of steroids. ${ }^{79}$ The optical density changes in the nucleus and the cortical regions have been found to be age-related. ${ }^{80}$ Aside from age and genetic ${ }^{81}$ factors being key reasons for progress towards cataract, reasons from which one can safeguard oneself, such as smoking, ${ }^{82}$ UV exposure, ${ }^{83}$ and obesity have been found to be responsible for development of cataract not only in the elderly but also in younger individuals. 
Since accelerated rise in the optical density of the ocular lens is considered a predictor of progression towards cataract, regular monitoring of the density levels of the lens is of vital importance. ${ }^{56}$ Hence, development of fast, easy, noninvasive, reliable methods for measuring lens density under clinical settings is necessary. ${ }^{84}$ Some examples of measurement techniques that are signal-based and directly measure the optical density of the lens are autofluorescence ${ }^{85}$ and reflectance techniques. ${ }^{86}$ However, these techniques are associated with serious drawbacks. The autofluorescence technique is expensive and the signal obtained is found to be heavily affected by scattering of light by the aging lens. ${ }^{38}$ Similarly the signal obtained by reflectance techniques is susceptible to being compromised by the presence of drusen especially in the aging retina. ${ }^{38}$ Psychophysical techniques on the other hand employ photopic or scotopic vision and involve minimal risk. Additionally one can neglect the loss of signal due to scattering of light by the aging lens.

Psychophysical techniques employing photopic vision are fast and easy but are dependent on the response of the associated cone cells in the retina. Popular methods employing photopic vision are heterochromatic flicker photometry $(\mathrm{HFP})^{87}$ and color matching techniques. ${ }^{88}$ These techniques employ the photopic luminous efficiency function which is directly dependent on the weighting of the long wavelength sensitive L-cones and medium wavelength sensitive M-cones. ${ }^{89}$ It is known that the relative weights of L-cones and the M-cones vary 
significantly amongst individuals. Therefore data for ocular parameters measured employing these techniques have been questioned for reliability. ${ }^{56}$

Scotopic vision, mediated by the rod cells, has been used by Wald in $1945^{14}$ and Wright in $1951^{90}$ to compute the lens density spectrum by comparing the scotopic sensitivity curves of phakic and aphakic individuals. The differences they reported were attributed to the transmittance of the crystalline lens in the phakic individuals. Further, the scotopic luminous efficiency functions of the aphakic individuals had a close resemblance to the rhodopsin absorption spectrum. Therefore a comparison between the rhodopsin absorbance spectrum and the scotopic luminous efficiency function at absolute thresholds of vision can be used to compute the lens density spectrum for phakic individuals. ${ }^{62}$ Testing at absolute thresholds necessitates prolonged periods of dark adaptation making the testing very lengthy. However, results obtained under such conditions are found to be consistent with those obtained by ex-vivo techniques. ${ }^{56}$ Rod mediated scotopic vision is dependent on the rhodopsin absorbance template, which is almost constant in individuals, unlike photopic vision with its dependence on the weighting of L-cone to M-cones. Hence to establish the reliability of LEA measurements by Mapcatsf, we compared the LEA for a group of individuals tested on Mapcatsf to their LEA values obtained at absolute scotopic thresholds after complete dark adaptation. A detailed description of the theory and testing procedure of lens density measurement by Mapcatsf can be found in chapter 2, section 2.2 .2 and 2.3 respectively. 


\section{Section 6.2}

\section{Scotopic Measurements: Theory}

Vision taking place under light conditions of -2.5 log Trolands falls in the category of scotopic vision and is mediated by rod cells. ${ }^{91}$ The spatial density distribution of the rods is such that the fovea can be considered a rod-free zone but there is an increase in rod density with increasing eccentricity from the fovea, reaching 190,000 rods $/ \mathrm{mm}^{2}$ at 20 to 30 degrees from the fovea. ${ }^{92}$ Taking into account that above 5 degrees eccentricity density of MP is negligibly small, the scotopic luminosity function above 5 degrees is affected only by the transmittance spectrum of the lens $T_{L}(\lambda)$ and the rhodopsin absorbance spectra $R(\lambda) .{ }^{93}$

$$
V_{\text {scotopic }}=R(\lambda) T_{L}(\lambda)
$$

$T_{L}(\lambda)$ was calculated for that of a 32 year old person using the van de Kraats and van Norren template and corrected for age by the template suggested by Sagawa and Takahashi. ${ }^{52}$ The absolute thresholds of vision were tested for a peripherally viewed stimulus illuminated by either a blue or a green LED, similar to those used for the perifoveal testing on Mapcatsf. The stimulus for the scotopic measurements was designed to be positioned at approximately the same retinal eccentricity as the periphery of the $15^{\circ}$ degree stimulus on Mapcatsf. The rationale was that any traces of MP at this eccentricity would affect each method of measurement equally. The similar LED sources and the eccentricity of the perifoveal stimulus used enabled direct comparison between the LEAs obtained 
by the two separate methods. The luminances of the stimulus due to the blue and the green LEDs, $L_{B}, L_{G}$ are mathematically expressed in Eq.s (2) and (3) respectively.

$$
\begin{aligned}
L_{B} & =\int B(\lambda) T_{F}(\lambda) T_{w}\left(x_{b}, \lambda\right) R(\lambda) T_{L}(\lambda, a) d \lambda \ldots \ldots \ldots \ldots \ldots . .(2) \\
L_{G} & =\int G(\lambda) T_{F}(\lambda) T_{w}\left(x_{g}, \lambda\right) R(\lambda) T_{L}(\lambda, a) d \lambda \ldots \ldots \ldots \ldots \ldots . .(3)
\end{aligned}
$$

The terms $B(\lambda), G(\lambda)$ denote the normalized spectra of the blue and the green LEDs respectively. The spectra of both the LEDs were obtained using the Ocean Optics ${ }^{\circledR}$ spectrometer. The transmittance of an auxiliary neutral density filter placed in the path of the testing light to maintain full scotopic viewing condition is denoted by $T_{F}(\lambda)$. The terms $T_{w}\left(x_{b}, \lambda\right), T_{w}\left(x_{g}, \lambda\right)$ are the transmittances of a variable density neutral wedge at the translational settings $x_{b}$ and $x_{g}$, at which the absolute scotopic thresholds are detected for the blue and the green LEDs respectively. Since, at absolute threshold, the luminance values are equal - both are essentially zero - we get:

$$
\frac{10^{-1.2036 x_{g}}}{10^{-1.1364 x_{b}}}=0.59 \frac{\int B(\lambda) T_{F}(\lambda) T_{w}\left(x_{b}, \lambda\right) R(\lambda) T_{L}(\lambda, a) d \lambda}{\int G(\lambda) T_{F}(\lambda) T_{w}\left(x_{g}, \lambda\right) R(\lambda) T_{L}(\lambda, a) d \lambda}
$$

The factors 1.2036, 1.1364 and 0.59 appearing in eq. (4) are the result of calibration of the variable density wedge and the LED sources. 
On observation, the right-hand side of Eq. (4) gives a unique value for a particular value of " $a$ ”. Therefore by varying the values of LEA, “ $a$ ”, we can generate values of the right-hand side. Labeling the left- hand side of Eq. 4 above as “ $F$ ”, we can obtain a plot of LEA with respect to F, and this is shown in Fig.6.1. The plot of LEA vs F shown in Fig. 5 was fitted with a function of the form given in Eq. 5 to enable computation of LEA from F.

$$
L E A=\frac{a+b F}{1+c F+D F^{2}}
$$

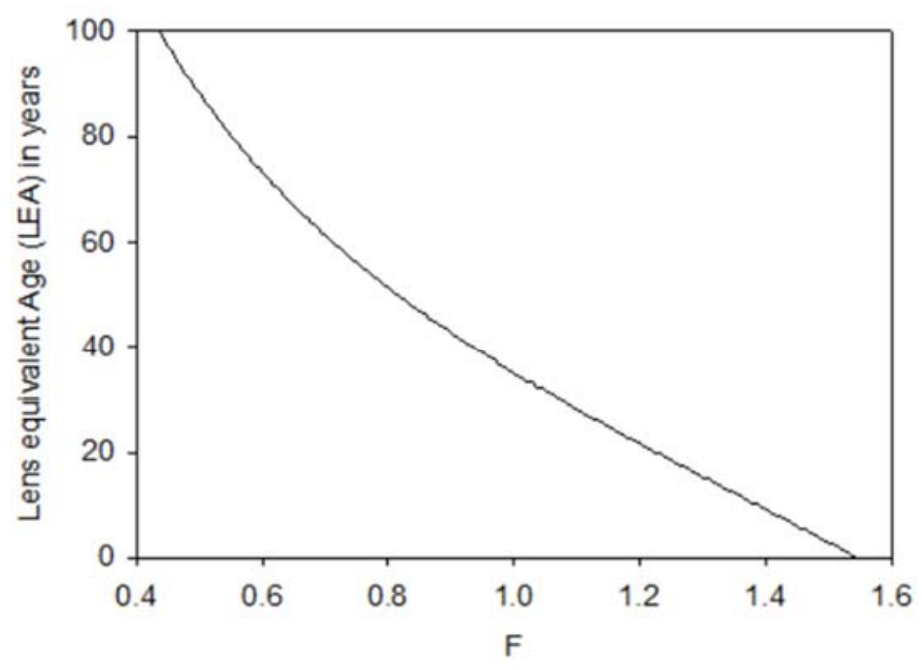

Fig.6.1 The polynomial fit to the plot of LEA with F. 


\section{Section 6.3}

\section{Scotopic Measurements: Instrument design}

The peripheral stimulus with the provision of central gaze fixation is shown in Fig. 6.2. The 0.5 degree stimulus is set 7.5 degrees eccentric to the central gaze position which is facilitated by a dimly lit red LED of 0.25 degrees radius. The stimulus in the periphery is illuminated by a blue LED of peak wavelength 455 $\mathrm{nm}$ or a green LED of peak wavelength $515 \mathrm{~nm}$. The input to the LEDs was provided by a frequency-controlled circuit so as to prevent variation in peak wavelengths with change of intensity. The driver associated with feeding the input signal to the LED generated pulses in the kilohertz range. The LEDs being cycled at $0.5 \mathrm{~s}$ "on" and $1.0 \mathrm{~s}$ "off” resulted in the visual perception of pulses of short duration.

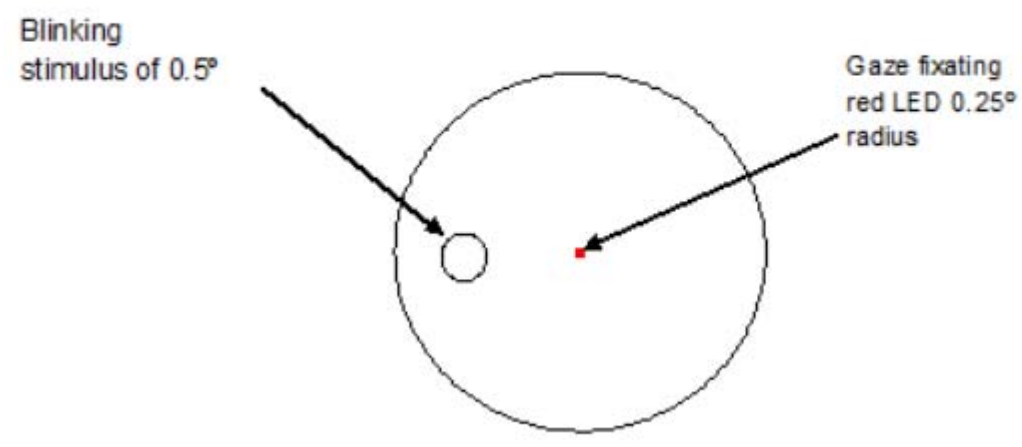

Fig. 6.2 The central gaze fixating stimulus used for absolute scotopic threshold measurements. 
The optical layout for the setup for scotopic measurement is shown in Fig 6.3. The sphere S acts in the capacity of an integrating sphere housing the test LEDs in the upper interior section, providing spatially uniform light emerging from the aperture. To maintain scotopic viewing conditions, a series of neutral density filters were placed in the light path between the sphere "S" and the eyepiece. The variable density wedge filter was translated electromechanically to alter the luminance levels of the test LEDs to reach the absolute scotopic threshold. The opaque screen to the left of the housing for the eyepiece consisted of precisely cut apertures defining the stimulus and the fixation target. Also posterior to the opaque screen, the dim red LED is placed to illuminate the fixation target. An achromatic doublet lens is positioned to the right end of the housing which can be moved back and forth in a telescopic arrangement. As explained in section 2.1 (Fig. 2.3), to ensure the maintenance of eccentricity at which the image of the stimulus is formed in the retina of the subject, a non-limiting viewing aperture is positioned so that the distance between the nodal point of the subject's eye and the achromatic doublet lens is equal to its focal length. ${ }^{43}$ 


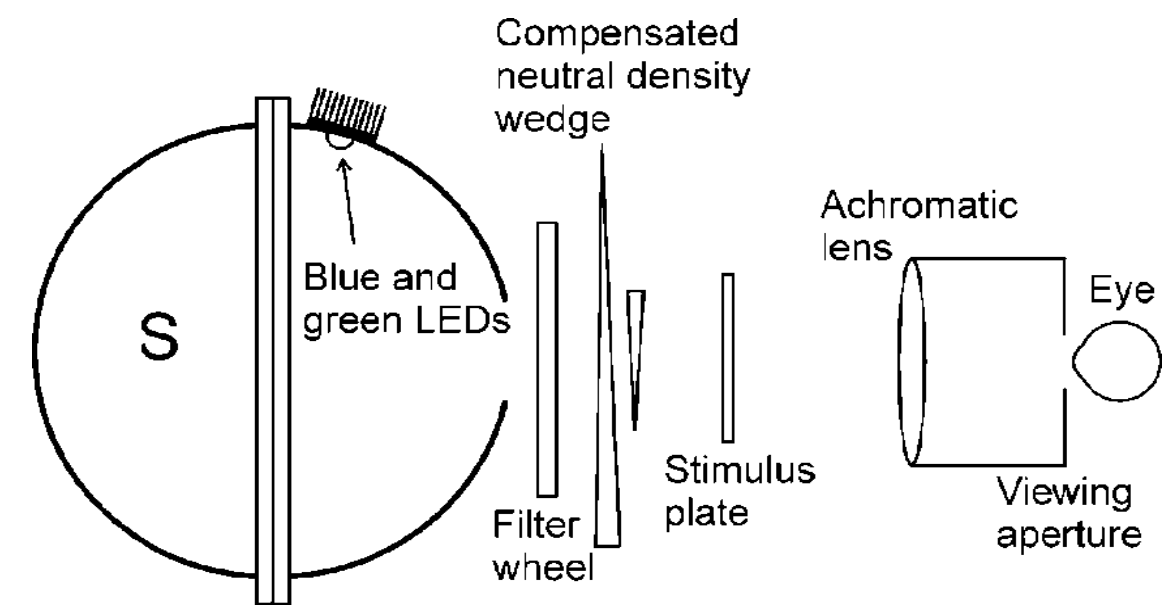

Fig. 6.3 The layout and optical setup for the scotopic lens density measurement

\section{Section 6.4}

\subsubsection{Design and Plan of the Study}

Twenty five subjects were recruited between the ages of 20 and 75 years (average age $39 \pm 14$ years) and were in possession of healthy vision. They were required to sign FIU-IRB approved informed consent.

Lens densities were measured for the subjects recruited by the two methods. Since the lens densities are usually similar in both the eyes, only one eye was tested. ${ }^{56}$

\subsubsection{Photopic Testing}

As the lens densities or LEA measurements by Mapcatsf are obtained from the perifoveal flicker null measurements, the testing procedure was the same as discussed in chapter 2 section 2.3, or in the section where the testing procedure is discussed in Bone and Mukherjee. ${ }^{43}$ 


\subsubsection{Scotopic Testing}

The subjects were fully dark-adapted for 30 minutes in a room of complete darkness. However, prior to dark adaptation, the eyepiece lens was adjusted to bring the fixation target and the peripheral stimulus into sharp focus. During the period of dark adaptation, a further precaution was taken to occlude the subject's test eye with an eyepatch. Once fully dark adapted, the operator turned the blinking blue stimulus "on" with the subject being directed to gaze at the minimally lit red LED. Such a direction from the operator ensured the blinking stimulus appeared in the periphery of the subject's viewing field. The wedge setting initially was set at a level such that the luminance of the stimulus was higher than at threshold. Consequently the subjects counted all 14 blinks that occurred during a 20 seconds measurement period. The intensity of the testing stimulus was lowered incrementally by translating the variable optical density wedge. The subject was allowed 20 seconds to report the number of blinks that were seen at each setting of the wedge. The test was terminated when the subject started reporting around 2 blinks for the span of 20 seconds. Subsequently the blue LED was replaced by the green LED and the procedure was repeated. 


\section{Section 6.5}

\section{Results:}

A scatter plot was generated between the number of counts and the wedge positions. A three parameter sigmoidal function shown in Eq. 6 below was fitted to the data.

$$
N=\frac{a}{1+e^{-\frac{\left(x-x_{o}\right)}{b}} \ldots \ldots . . .}
$$

In Eq. 6 " $N$ " is the number of counts and the variable $\mathrm{x}$ depicts the variable density wedge position. A $50 \%$ visibility criterion was used for obtaining the wedge setting at absolute scotopic threshold. The criterion reflects the setting at which $50 \%$ of the blinks were visible. The values of threshold settings obtained from Eq. 6 were substituted in the left-hand side of Eq. 4 to obtain the quantity F which, when substituted in Eq.5, gave us the LEA. The uncertainties in measurement of the scotopic LEAs were associated with the uncertainty generated for the parameter $x_{o}$ in Eq.6 on Sigmaplot. 


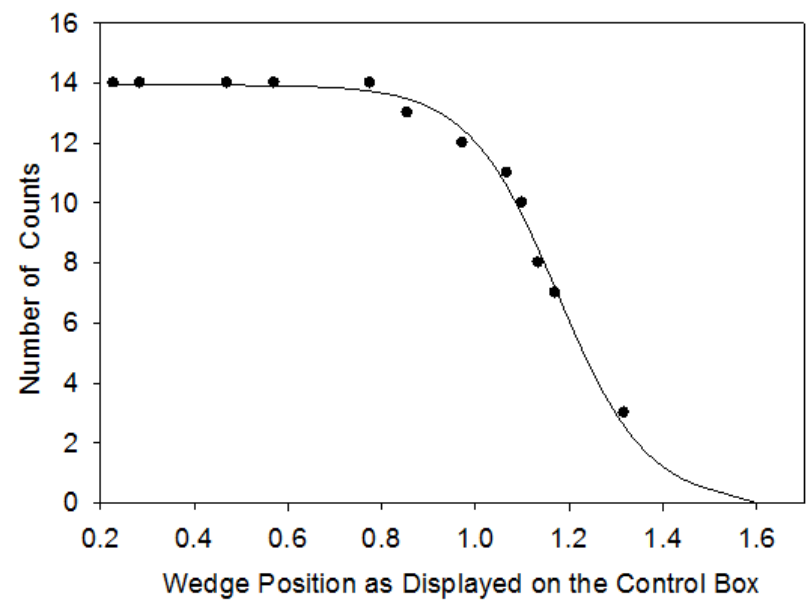

Fig 6.4 A sample scatter plot obtained for a participating subject for one of the LEDs tested. The inverse sigmoidal curve of the form as given in Eq.(6) was fitted to obtain the absolute scotopic threshold at the $50 \%$ viewing criterion.

The scatter plot for the photopic LEA versus the scotopic LEA is shown in Fig. 4.5. The error bars associated with the data points are the standard errors in measurement of LEAs under the two separate viewing conditions respectively.

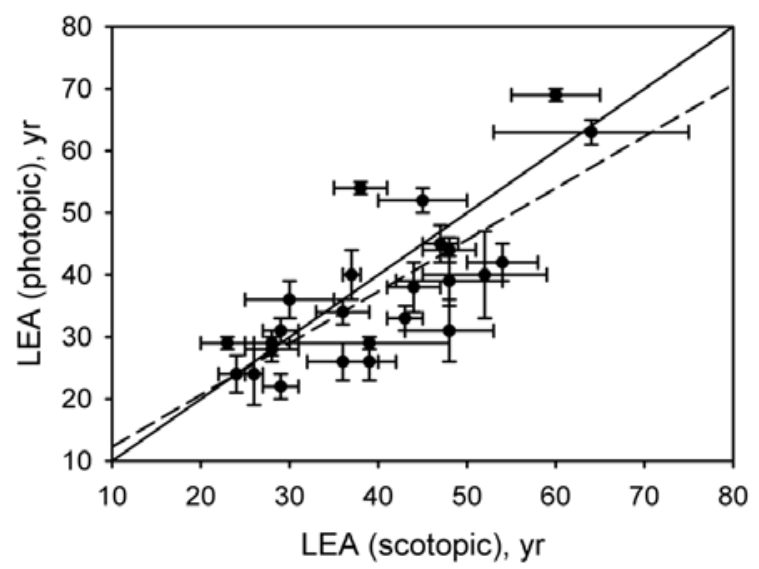

Fig 6.5 The scatter plot of photopic LEAs obtained from Mapcatsf versus the scotopic LEAs obtained at absolute threshold. The solid line is of slope unity. The dashed line is the regression line of Eq. $y=0.833 x+3.945$. 
The solid line of slope unity represents equality between the LEAs obtained under the two separate viewing conditions. Results of regression analysis of the scatter plot in Fig 4.5 gave a slope of 0.8333 and an $r^{2}$ value of 0.59 with $p<0.0001$.To further analyze the gathered data for systematic deviations, we performed a Bland Altman analysis as shown in Fig. 6.6. The mean difference between the photopic and the scotopic LEAs was 2.00 years with 8.68 years being the 95 percent confidence intervals.

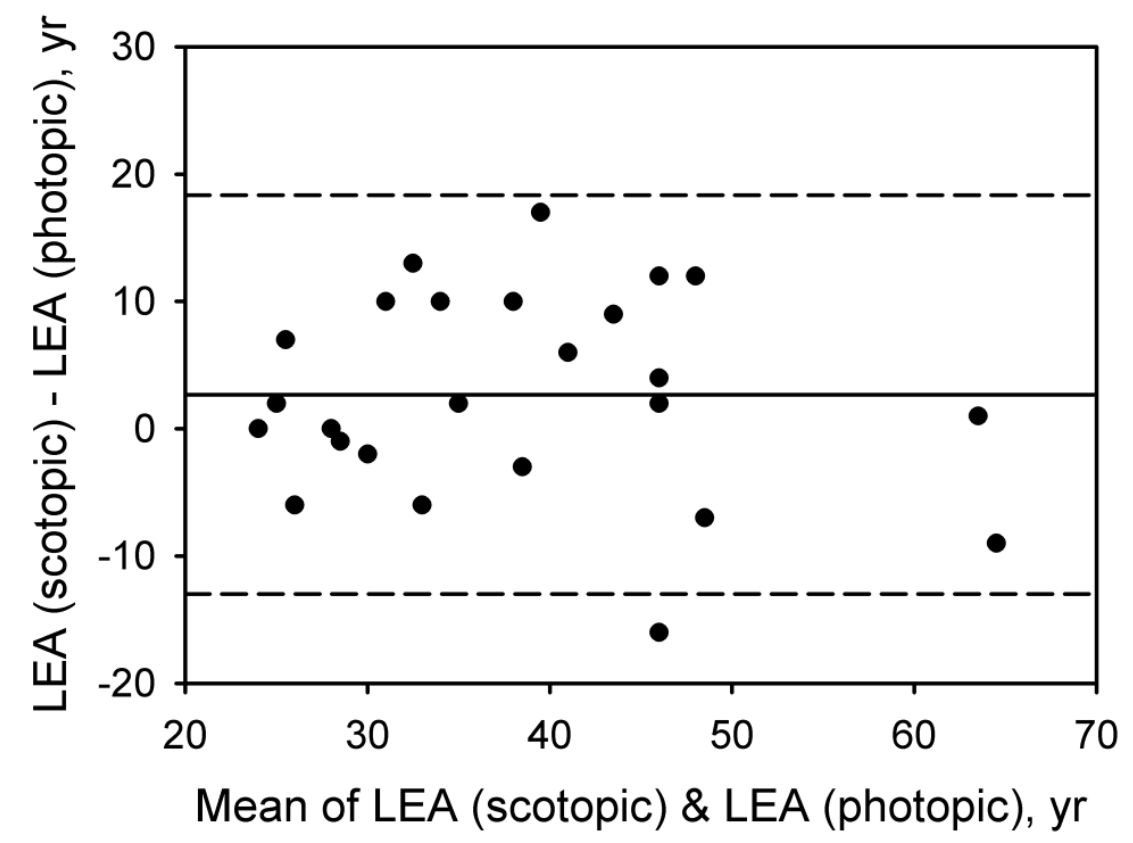

Fig 6.6 The Bland Altman plot used for testing for levels of agreement of the results between the LEAs obtained under the two separate viewing conditions. The mean difference which was 2.00 years is depicted by the solid horizontal line and the dashed lines are for depicting Mean $\pm 2 \mathrm{SD}$. 


\section{Section 6.6}

\section{Discussion}

An overall good level of agreement between the LEAs measured by the two methods can be surmised from the slope of $0.833 \pm 0.144$ and the intercept of 3.945 years of the regression line in Fig.6.5. The average difference between the photopic and the scotopic LEAs being 2 years with the limits of agreement being 8.0 years further supports our findings.

Wooten et $\mathrm{al}^{56}$ had reported a slope of 0.92 and an intercept of 0.09 density units for the regression line for a scatter plot of lens densities obtained under photopic and absolute scotopic viewing conditions. Our measurements were in terms of LEA, but LEA is proportional to the optical density of the lens. Therefore we can conclude that our results of regression analysis of Fig.6.5 compare favorably with Wooten et al 2007. These researchers had proposed an alternate approach of testing involving vision mediated by the rod cells, but only necessitating partial dark adaptation. However they had suggested the possibility of some participation of cone cells under such conditions. Therefore to avoid any cone intrusion, the absolute scotopic thresholds were tested after complete dark adaptation.

Mapcatsf uses an age-modified $10^{\circ}$ photopic luminous efficiency function. Photopic vision is dependent chiefly on the responses of the L-(long) and M(medium) wavelength cones, and the ratio of the L- to M-cones is known to vary over a wide range among individuals. Some of the reported ranges of variation in 
L and M cone ratios are 0.6 to 12 by Carroll et al., ${ }^{61} 1.1$ to 16.5 by Hofer et al., ${ }^{60}$ and 0.47 to 15.82 by Sharpe et al..$^{89}$ The standard CIE $10^{\circ}$ luminous efficiency function is based on an average L to M cone weight. Hence measurement of LEA employing photopic methods can be influenced by the variation in $\mathrm{L}$ and $\mathrm{M}$ cones among individuals. To investigate the effect of the variation in cone weights on the LEAs measured by Mapcatsf, we used the template developed by Sharpe and Stockman ${ }^{89}$ which enables constructing the $10^{\circ}$ luminous efficiency function for any value of the $\mathrm{L}$ to $\mathrm{M}$ cone ratio. The ratio of photodetector intensities provided by each subject allowed the LEA to be computed from Eq. (11) in section 2.2.2. The only modification involved in the present case was replacing the standard age-modified luminous efficiency function by a similar function at the extreme cone weights of 0.47 and 16.5. For the extreme cone weights, the lower extreme was the one reported by Sharpe and Stockman and the upper extreme value was the one reported by Hofer et al. Table 6.1 depicts the results of the analysis. 


\begin{tabular}{|c|c|c|c|c|c|}
\hline $\begin{array}{c}\text { Subject } \\
\text { No. }\end{array}$ & $\begin{array}{c}\text { Age } \\
\text { (yrs.) }\end{array}$ & $\begin{array}{l}\text { Measured } \\
\text { scotopic } \\
\text { LEA } \pm \text { SD } \\
\text { (yrs.) }\end{array}$ & $\begin{array}{l}\text { Measured } \\
\text { photopic } \\
\text { LEA } \pm \text { SD } \\
\text { (yrs.) }\end{array}$ & $\begin{array}{l}\text { Photopic } \\
\text { LEA } \pm \text { SD } \\
\text { for L:M } \\
\text { weight of } \\
0.47(\text { yrs. }\end{array}$ & $\begin{array}{l}\text { Photopic } \\
\text { LEA } \pm \text { SD } \\
\text { for L:M } \\
\text { weight of } \\
16.5(\text { yrs. }\end{array}$ \\
\hline 1 & 71 & $60+/-5$ & $69+/-1$ & $75+/-1$ & $66+/-1$ \\
\hline 2 & 34 & $43+/-2$ & $33+/-2$ & $41+/-2$ & $32+/-2$ \\
\hline 3 & 24 & $29+/-2$ & $31+/-2$ & $39+/-2$ & $30+/-2$ \\
\hline 4 & 28 & $37+/-1$ & $40+/-4$ & $43+/-4$ & $34+/-4$ \\
\hline 5 & 28 & $28+/-3$ & $28+/-2$ & $31+/-1$ & $22+/-1$ \\
\hline 6 & 40 & $47+/-2$ & $45+/-3$ & $49+/-3$ & $39+/-3$ \\
\hline 7 & 27 & $52+/-7$ & $40+/-7$ & $44+/-8$ & $34+/-8$ \\
\hline 8 & 40 & $48+/-6$ & $39+/-4$ & $44+/-5$ & $33+/-4$ \\
\hline 9 & 30 & $39+/-9$ & $29+/-1$ & $37+/-2$ & $25+/-2$ \\
\hline 10 & 36 & $48+/-5$ & $31+/-5$ & $34+/-4$ & $25+/-4$ \\
\hline 11 & 60 & $45+/-5$ & $52+/-2$ & $57+/-2$ & $47+/-2$ \\
\hline 12 & 61 & $64+/-11$ & $63+/-2$ & $68+/-2$ & $58+/-2$ \\
\hline 13 & 53 & $48+/-3$ & $44+/-2$ & $48+/-3$ & $40+/-3$ \\
\hline 14 & 28 & $23+/-3$ & $29+/-1$ & $33+/-1$ & $22+/-2$ \\
\hline 15 & 26 & $39+/-3$ & $26+/-3$ & $29+/-3$ & $20+/-3$ \\
\hline 16 & 24 & $36+/-4$ & $26+/-3$ & $29+/-5$ & $20+/-5$ \\
\hline 17 & 36 & $30+/-5$ & $36+/-3$ & $45+/-2$ & $35+/-2$ \\
\hline 18 & 32 & $44+/-3$ & $38+/-4$ & $42+/-4$ & $32+/-4$ \\
\hline 19 & 28 & $26+/-1$ & $24+/-5$ & $29+/-4$ & $18+/-4$ \\
\hline 20 & 29 & $24+/-2$ & $24+/-3$ & $29+/-4$ & $20+/-4$ \\
\hline 21 & 28 & $28+/-3$ & $29+/-2$ & $33+/-3$ & $23+/-2$ \\
\hline 22 & 61 & $38+/-3$ & $54+/-1$ & $57+/-2$ & $47+/-2$ \\
\hline 23 & 46 & $36+/-3$ & $34+/-2$ & $37+/-2$ & $27+/-2$ \\
\hline 24 & 28 & $29+/-2$ & $22+/-2$ & $28+/-3$ & $17+/-3$ \\
\hline 25 & 51 & $54+/-4$ & $42+/-3$ & $44+/-2$ & $33+/-2$ \\
\hline
\end{tabular}

Table 6.1. LEAs measured under the two separate viewing conditions are presented. The columns 5 and 6 consists the recalculated LEAs at extreme cone weights of 0.47 and 16.5 respectively.

A two tailed Student's t-test was conducted at the 0.05 confidence level to test the significance of difference between the LEAs measured by Mapcatsf and at absolute scotopic threshold. Such a testing resulted in 14 subjects having a significant difference in their LEAs. For these 14 subjects the Student's t-test was 
repeated, but their photopic LEA value was selected at the extreme cone weight, which placed it closer to the LEA at absolute scotopic threshold. With this modification, significant differences remained for 6 of the subjects. These 6 subjects were subjects $1,10,15,16,22$ and 25 . Therefore we can say that variability in cone weight cannot completely account for the differences observed between scotopic and the photopic LEAs.

As a concluding perspective, the results of LEAs measured under the two separate viewing conditions are in reasonable agreement with previously published results. ${ }^{56}$ Testing under scotopic conditions necessitates long periods of dark adaptation especially if testing is preferred at absolute threshold conditions. Therefore the rapid testing under photopic conditions would be recommended as the preferable choice for routine measurements. Mapcatsf truly stands out as a reliable instrument capable of measuring both MPOD and LOD/LEA with a high level of repeatability for clinical purposes. 


\section{Chapter 7}

\section{Credibility of Mapcatsf for Monitoring MPOD levels of an Individual Consuming Lutein or Its Derivative Supplement for Six Months.}

\section{Section 7.1}

\section{Introduction:}

Macular pigment being composed of xanthophylls, lutein, zeaxanthin and mesozeaxanthin, ${ }^{94,95,12}$ has been inferred to act in a protective capacity against photooxidative stress, ${ }^{96,97,} 98$ making it a vantage arena of research for vision scientists in the past few decades. ${ }^{99,100,101,102}$

The level of MP possessed by individuals when measured by in-vivo methods is expressed in terms of optical density (OD) ${ }^{102}$ or signal counts. ${ }^{103}$ A wide range of variability in OD across individuals has been reported, ${ }^{104}$ along with the evidence that individuals with higher levels of MPOD are at a lower risk of developing AMD, ${ }^{105,106,107}$ one of the leading causes of vision impairment affecting senior individuals. ${ }^{108}$ The component carotenoids of MP are not produced by the human body but can be assimilated through diet rich in them ${ }^{101,}{ }^{109}$ or via supplements containing such carotenoids. ${ }^{110,111}$

Bone and Landrum have been pioneers in the arena of carotenoid research to show that not only can supplements be used to elevate levels of MP but also levels of MP reached at the end of the supplement consumption are not altered significantly over the course of time. ${ }^{112}$ Their research involved monitoring levels 
of MPOD at regular intervals by using heterochromatic flicker photometry (HFP) in individuals consuming lutein.

Similarly, researchers or clinical practitioners, pursuing supplement studies, primarily measure MPOD at regular intervals in individuals consuming the supplement over the entire tenure of the study. ${ }^{113,114}$ Therefore developing instrumentation methods providing a high level of precision is important in order to properly monitor changes in the levels of MPOD during the course of such studies.

HFP is one of the preferred means of measuring MPOD by researchers. The primary intention of developing Mapcatsf, which is a novel LED-based HFP instrument, has been to analyze and endorse its repeatability and credibility for measuring and monitoring MPOD under clinical settings.

As reported in chapter 3, preliminary studies with 83 subjects have shown good repeatability. However the credibility of Mapcatsf as an effective instrument for use in clinical settings is further established by inferring its capacity to monitor changing levels of MPOD in individuals. Such an attribute is established in this chapter where we analyzed the MPOD values measured at regular intervals for subjects consuming either lutein or the ester derivative of all the component carotenoids making up MP over the course of six months. 


\section{Section 7.2}

\section{Overview of the Supplements:}

For the sake of comparison of responses in the levels of MPOD reached at the end of six months, we used either of two supplements. Since there has been published evidence that lutein and zeaxanthin are the ingredients that can significantly boost the levels of MPOD in an individual, ${ }^{115,110}$ a supplement comprising primarily lutein was chosen as one of the supplements and was labeled as "L". The lutein supplement used was derived from marigolds and was in the form of gel-cap capsules with each capsule being $20 \mathrm{mg}$ by dosage, and consisting of $95 \%$ lutein and 5\% zeaxanthin.

The other supplement was an ester derivative of lutein, zeaxanthin and mesozeaxanthin which was labeled as "MicroMic", manufactured by IOSA SA de CV. The "MicroMic" supplement was also in the form of a gel-cap capsule and consisted of $7 \mathrm{mg}$ of lutein, $7 \mathrm{mg}$ of zeaxanthin and $7 \mathrm{mg}$ of meso- zeaxanthin.This supplement was tried since there has been evidence of such ester derivatives used in chicken feed enhancing the coloration of poultry ${ }^{116}$ and egg yolk ${ }^{116}$. This observation led to the hypothesis that it may be effective as a supplement to boost the levels of the MP in humans. 


\section{Section 7.3}

\section{Subject recruitment, profile and elimination criteria:}

There were 32 subjects recruited for the study. Individuals recruited were in possession of good ocular health and had to sign an FIU-IRB-approved informed consent. To check the effect of age on physiological response to intake of supplements, the total recruitment was divided into two age groups. One age group consisted of 18 subjects between the ages of 18 and 30 years (younger group) and the other group had 14 individuals of 50 years or older. Both the age groups included males and females. The younger age group comprised 7 males and 11 females of $23 \pm 3$ (Mean \pm SD) years. The older age group consisted of 9 males and 5 females of $58 \pm 8$ (Mean \pm SD) years. Due to the established evidence of smokers having a $\beta$-carotene-rich diet being at higher risk of developing

pulmonary cancer, ${ }^{117}$ care was taken that none of the subjects were smokers or had a history of smoking. Further, caution was taken not to involve women who were pregnant or had the likelihood of getting pregnant during the course of the study.

\section{Section 7.4}

\section{Design of Study}

The study was designed for a total of 24 weeks. The subjects' MPOD values were measured on Mapcatsf to provide the reference baseline value. They were then provided with the supplements and the MPOD values were subsequently measured at 8 week intervals for a total of 4 times during the entire duration of the 
study. The subjects were directed to consume one gel cap a day. They were further instructed that if they missed the pill for one day, they could consume two pills the following day. A printed schedule was also provided that had the followup dates when the subjects were required to get their MPOD tested.

\section{Section 7.5}

\section{Testing Procedure}

For a detailed overview of the testing procedure please refer to section 2.3.

\section{Section 7.6}

\section{MPOD levels at 8 weeks interval}

For each scheduled session, the entire test on Mapcatsf was conducted at least twice, with each test involving 5 foveal and five perifoveal measurements. The microprocessor associated with Mapcatsf computes the MPOD and the standard error in the mean for each test. Since the testing for MPOD was done a minimum of two times, with each measurement being independent of the other, the weighted average of the tests and the standard error in the mean were obtained from the formula:

$$
\bar{x} \pm \delta \bar{x}=\frac{\sum_{i} w_{i} x_{i}}{\sum_{i} w_{i}} \pm\left(\sum_{i} w_{i}\right)^{-1 / 2}
$$


where

$$
w_{i}=1 /\left(\delta x_{i}\right)^{2}
$$

The terms $x_{i}$ and $\delta x_{i}$ are the MPOD values and the errors associated with it for the $\mathrm{i}^{\text {th }}$ measurement, and the summation runs from 1 to $N$, the total number of tests. As each measurement was independent from the other, a Gaussian distribution could be assumed. The value of $\chi^{2}=\sum w_{i}\left(\bar{x}-x_{i}\right)^{2}$ was calculated and its ratio to $N-1$ was computed and compared to unity. The value $\mathrm{N}-1$ is considered the expectation value of $\chi^{2}$. If the ratio $\chi^{2} / N-1$ was found to be less than one, we accepted the standard error of the mean, $\delta x$, as shown in Eq. 1 . If it was larger than one, we calculated the factor $\mathrm{S}$ and increased the error $\delta x$ in Eq. (1) by a scale factor $S=\left[\chi^{2} / N-1\right]^{1 / 2}$. This procedure is described in the article 1975 by A.H. Rosenfeld in the “Annual Review of Nuclear Science” volume 25 page 555 .

The data obtained for the subjects in each category based on gender and age are presented in a tabular form. In tables 7.6.1 and 7.6.2, we present the value of the MPODs recorded for the period of 24 weeks for male and female subjects respectively in the younger age group. 


\begin{tabular}{|c|c|c|c|c|c|}
\hline $\begin{array}{c}\text { Subject } \\
\text { No. }\end{array}$ & $\begin{array}{c}\text { Age } \\
\text { (yrs })\end{array}$ & $\begin{array}{c}\text { Baseline } \\
(\text { MPOD } \pm \text { SEM })\end{array}$ & $\begin{array}{c}\text { Week 8 } \\
(\text { MPOD } \pm \text { SEM })\end{array}$ & $\begin{array}{c}\text { Week 16 } \\
(\text { MPOD } \pm \text { SEM })\end{array}$ & $\begin{array}{c}\text { Week 24 } \\
\text { (MPOD } \pm \text { SEM) }\end{array}$ \\
\hline L1 & 24 & $0.882 \pm 0.011$ & $0.874 \pm 0.012$ & $0.830 \pm 0.022$ & $0.928 \pm 0.014$ \\
\hline L3 & 22 & $0.290 \pm 0.006$ & $0.310 \pm 0.006$ & $0.344 \pm 0.007$ & $0.355 \pm 0.020$ \\
\hline L4 & 22 & $0.367 \pm 0.012$ & $0.427 \pm 0.008$ & $0.507 \pm 0.012$ & $0.514 \pm 0.013$ \\
\hline L5 & 18 & $0.209 \pm 0.016$ & $0.228 \pm 0.007$ & $0.244 \pm 0.012$ & $0.277 \pm 0.006$ \\
\hline L9 & 20 & $0.528 \pm 0.010$ & $0.476 \pm 0.015$ & $0.554 \pm 0.011$ & $0.598 \pm 0.013$ \\
\hline L10 & 25 & $0.603 \pm 0.020$ & $0.678 \pm 0.021$ & $0.627 \pm 0.006$ & $0.679 \pm 0.008$ \\
\hline M3 & 30 & $0.322 \pm 0.015$ & $0.316 \pm 0.006$ & $0.338 \pm 0.009$ & $0.3806 \pm 0.009$ \\
\hline
\end{tabular}

Table 7.6.1 The baseline and measured MPODs at 8 weeks interval of male subjects between 18 and 30 years during the supplementation period. The subjects on "Lutein" are titled as "L" and the one provided the "Micromic" are titled "M". 


\begin{tabular}{|c|c|c|c|c|c|}
\hline $\begin{array}{c}\text { Subject } \\
\text { No. }\end{array}$ & $\begin{array}{c}\text { Age } \\
\text { (yrs. }\end{array}$ & $\begin{array}{l}\text { Baseline } \\
\text { (MPOD } \pm \text { SEM) }\end{array}$ & $\begin{array}{c}\text { Week 8 } \\
(\text { MPOD } \pm \text { SEM) }\end{array}$ & $\begin{array}{c}\text { Week 16 } \\
(\text { MPOD } \pm \text { SEM })\end{array}$ & $\begin{array}{c}\text { Week 24 } \\
\text { (MPOD } \pm \text { SEM) }\end{array}$ \\
\hline L2 & 22 & $0.641 \pm 0.011$ & $0.631 \pm 0.018$ & $0.594 \pm 0.007$ & $0.597 \pm 0.009$ \\
\hline L6 & 22 & $0.614 \pm 0.010$ & $0.653 \pm 0.008$ & $0.707 \pm 0.017$ & $0.680 \pm 0.008$ \\
\hline L8 & 29 & $0.251 \pm 0.011$ & $0.299 \pm 0.008$ & $0.359 \pm 0.025$ & $0.320 \pm 0.0231$ \\
\hline L11 & 20 & $0.231 \pm 0.008$ & $0.201 \pm 0.008$ & $0.239 \pm 0.015$ & $0.242 \pm 0.014$ \\
\hline L12 & 22 & $0.488 \pm 0.014$ & $0.537 \pm 0.013$ & $0.536 \pm 0.022$ & $0.589 \pm 0.017$ \\
\hline M1 & 22 & $0.335 \pm 0.014$ & $0.377 \pm 0.012$ & $0.397 \pm 0.007$ & $0.446 \pm 0.005$ \\
\hline M2 & 24 & $0.184 \pm 0.014$ & $0.146 \pm 0.011$ & $0.153 \pm 0.009$ & $0.172 \pm 0.012$ \\
\hline M4 & 29 & $0.550 \pm 0.012$ & $0.581 \pm 0.016$ & $0.563 \pm 0.010$ & $0.598 \pm 0.013$ \\
\hline M5 & 22 & $0.237 \pm 0.008$ & $0.282 \pm 0.007$ & $0.304 \pm 0.004$ & $0.334 \pm 0.008$ \\
\hline M6 & 23 & $0.198 \pm 0.018$ & $0.216 \pm 0.005$ & $0.237 \pm 0.008$ & $0.255 \pm 0.009$ \\
\hline M7 & 22 & $0.210 \pm 0.013$ & $0.284 \pm 0.007$ & $0.347 \pm 0.015$ & $0.347 \pm 0.009$ \\
\hline
\end{tabular}

Table 7.6.2 The baseline and measured MPODs at 8 weeks intervals of female subjects between 18 and 30 years during the supplementation period. The subjects on "Lutein" are titled as "L" and the ones provided the "Micromic" are titled "M".

Tables 7.6.3 and 7.6.4 represent the data obtained for the male and the female subjects respectively in the older age groups. 


\begin{tabular}{|c|l|l|l|l|l|}
\hline $\begin{array}{c}\text { Subject } \\
\text { No. }\end{array}$ & $\begin{array}{l}\text { Age } \\
(\mathrm{yrs} .)\end{array}$ & $\begin{array}{l}\text { Baseline } \\
(\mathrm{MPOD} \pm \mathrm{SEM})\end{array}$ & $\begin{array}{l}\text { Week 8 } \\
(\mathrm{MPOD} \pm \mathrm{SEM})\end{array}$ & $\begin{array}{l}\text { Week 16 } \\
(\mathrm{MPOD} \pm \mathrm{SEM})\end{array}$ & $\begin{array}{l}\text { Week 24 } \\
(\mathrm{MPOD} \pm \mathrm{SEM})\end{array}$ \\
\hline M13 & 51 & $0.757 \pm 0.008$ & $0.765 \pm 0.014$ & $0.810 \pm 0.008$ & $0.807 \pm 0.010$ \\
\hline M15 & 61 & $0.601 \pm 0.006$ & $0.587 \pm 0.007$ & $0.558 \pm 0.006$ & $0.597 \pm 0.015$ \\
\hline L15 & 70 & $0.786 \pm 0.006$ & $0.803 \pm 0.007$ & $0.810 \pm 0.005$ & $0.776 \pm 0.008$ \\
\hline L16 & 61 & $0.430 \pm 0.010$ & $0.407 \pm 0.006$ & $0.402 \pm 0.012$ & $0.461 \pm 0.062$ \\
\hline L17 & 52 & $0.559 \pm 0.024$ & $0.564 \pm 0.013$ & $0.617 \pm 0.018$ & $0.586 \pm 0.012$ \\
\hline L18 & 51 & $0.505 \pm 0.008$ & $0.519 \pm 0.015$ & $0.518 \pm 0.007$ & $0.573 \pm 0.010$ \\
\hline L19 & 53 & $0.604 \pm 0.009$ & $0.607 \pm 0.007$ & $0.665 \pm 0.030$ & $0.664 \pm 0.012$ \\
\hline L20 & 52 & $0.664 \pm 0.010$ & $0.666 \pm 0.009$ & $0.714 \pm 0.014$ & $0.701 \pm 0.103$ \\
\hline L22 & 71 & $0.488 \pm 0.014$ & $0.537 \pm 0.013$ & $0.537 \pm 0.023$ & $0.589 \pm 0.017$ \\
\hline
\end{tabular}

Table 7.6.3. The baseline and measured MPODs at 8 weeks interval of male subjects 50 years or above during the supplementation period. The subjects on "Lutein" are titled as "L" and the ones provided the "Micromic" are titled "M".

\begin{tabular}{|l|l|l|l|l|l|}
\hline $\begin{array}{l}\text { Subject } \\
\text { No. }\end{array}$ & $\begin{array}{l}\text { Age } \\
(\text { yrs. })\end{array}$ & $\begin{array}{c}\text { Baseline } \\
(\text { MPOD } \pm \text { SEM })\end{array}$ & $\begin{array}{c}\text { Week 8 } \\
(\text { MPOD } \pm \text { SEM })\end{array}$ & $\begin{array}{l}\text { Week 16 } \\
(\text { MPOD } \pm \text { SEM })\end{array}$ & $\begin{array}{l}\text { Week 24 } \\
(\text { MPOD } \pm S E M)\end{array}$ \\
\hline M14 & 55 & $0.442 \pm 0.009$ & $0.432 \pm 0.008$ & $0.433 \pm 0.009$ & $0.474 \pm 0.012$ \\
\hline L13 & 63 & $0.303 \pm 0.025$ & $0.321 \pm 0.011$ & $0.331 \pm 0.018$ & $0.311 \pm 0.017$ \\
\hline L21 & 51 & $0.224 \pm 0.010$ & $0.241 \pm 0.009$ & $0.264 \pm 0.006$ & $0.291 \pm 0.021$ \\
\hline L23 & 50 & $0.314 \pm 0.011$ & $0.378 \pm 0.013$ & $0.300 \pm 0.018$ & $0.272 \pm 0.012$ \\
\hline L24 & 53 & $0.448 \pm 0.009$ & $0.489 \pm 0.009$ & $0.469 \pm 0.009$ & $0.486 \pm 0.018$ \\
\hline
\end{tabular}

Table 7.6.4 The baseline and measured MPODs at 8 weeks interval of female subjects 50 years or above during the supplementation period. The subjects on "Lutein" are titled as "L" and the ones provided the "Micromic" are titled "M". 


\section{Section 7.7}

\section{Response to the supplementation}

To understand and compare the levels of responses of the subjects to the two types of supplements used in the study, we performed the following analyses presented in tables 7.7.1-7.7.4. We calculated the overall change in the MPOD, which is the difference between the final weighted averages of the MPOD associated with the weighted standard errors at the end of the 24 weeks and the initial values of MPOD associated with the weighted standard errors labeled as baseline. The percentage change in the MPOD levels at the end of the study was also calculated along with the associated standard error of the mean. The weighted values of MPOD obtained at 8 weeks intervals were plotted with respect to the week number. The weighted slope of the regression line for the plot gave us the rate of change of MPOD. The percentage compliance was calculated by obtaining from the subjects the number of pills left on the day of the final measurement and is represented as the percentage of the total number of pills consumed. 


\begin{tabular}{|c|c|c|c|c|c|c|c|}
\hline $\begin{array}{c}\text { Subject } \\
\text { No. }\end{array}$ & $\begin{array}{l}\text { Age } \\
\text { (yrs.) }\end{array}$ & \multicolumn{2}{|c|}{$\begin{array}{l}\text { Changes in } \\
\text { MPODand } \\
\text { SEM(d.u) }\end{array}$} & \multicolumn{2}{l|}{$\begin{array}{l}\text { \% Change in } \\
\text { MPOD and SEM }\end{array}$} & $\begin{array}{l}\text { Rate of } \\
\text { change of } \\
\text { MPOD } \\
\text { (d.u.)/week }\end{array}$ & $\begin{array}{l}\text { Percentage } \\
\text { Compliance }\end{array}$ \\
\cline { 2 - 7 } & & $\begin{array}{l}\text { MPOD } \\
\text { change }\end{array}$ & $\begin{array}{l}\text { SEM } \\
\text { change }\end{array}$ & $\begin{array}{l}\text { \% change } \\
\text { in MPOD }\end{array}$ & $\begin{array}{l}\text { SEM in } \\
\text { \% change }\end{array}$ & & \\
\hline L1 & 24 & 0.046 & 0.018 & 5.24 & 2.03 & 0.001 & 88.2 \\
\hline L3 & 22 & 0.065 & 0.021 & 22.29 & 7.23 & 0.003 & 93.6 \\
\hline L4 & 22 & 0.148 & 0.018 & 40.27 & 4.98 & 0.006 & 92.4 \\
\hline L5 & 18 & 0.069 & 0.017 & 32.90 & 8.64 & 0.003 & 99.4 \\
\hline L9 & 20 & 0.074 & 0.016 & 13.34 & 3.16 & 0.003 & 99.4 \\
\hline L10 & 25 & 0.076 & 0.012 & 12.65 & 3.53 & 0.003 & 81.1 \\
\hline M3 & 30 & 0.058 & 0.018 & 18.16 & 5.71 & 0.003 & 91.8 \\
\hline
\end{tabular}

Table 7.7.1 Response of male subjects in the younger age group to the supplement consumption considering the baseline and final MPOD values from table 7.6.1. The rate of change involves all the interim measurements done during the 6 month study. 


\begin{tabular}{|l|l|l|l|l|l|l|l|}
\hline $\begin{array}{l}\text { Subject } \\
\text { No. }\end{array}$ & $\begin{array}{l}\text { Age } \\
\text { (yrs.) }\end{array}$ & \multicolumn{2}{|c|}{$\begin{array}{r}\text { Changes in } \\
\text { MPOD and } \\
\text { SEM(d.u) }\end{array}$} & \multicolumn{2}{|c|}{$\begin{array}{r}\text { \% Change in } \\
\text { MPOD and SEM }\end{array}$} & $\begin{array}{l}\text { Rate of } \\
\text { change of } \\
\text { MPOD } \\
\text { (d.u.)/week }\end{array}$ & $\begin{array}{l}\text { Percentage } \\
\text { Compliance }\end{array}$ \\
\cline { 2 - 7 } & & $\begin{array}{l}\text { MPOD } \\
\text { change }\end{array}$ & $\begin{array}{l}\text { SEM } \\
\text { change }\end{array}$ & $\begin{array}{l}\text { \% change } \\
\text { in MPOD }\end{array}$ & $\begin{array}{l}\text { SEM in \% } \\
\text { change }\end{array}$ & & \\
\hline L6 & 22 & -0.043 & 0.014 & -6.79 & 2.27 & -0.002 & 93.6 \\
\hline L8 & 22 & 0.066 & 0.014 & 10.71 & 2.23 & 0.003 & 100 \\
\hline L11 & 20 & 0.011 & 0.017 & 4.83 & 7.24 & 0.0004 & 93.6 \\
\hline L12 & 22 & 0.008 & 0.014 & 1.39 & 2.31 & 0.0005 & 100 \\
\hline M1 & 22 & 0.110 & 0.015 & 32.90 & 4.69 & 0.005 & 86.9 \\
\hline M2 & 24 & -0.013 & 0.019 & -6.85 & -10.09 & -0.0002 & 98.2 \\
\hline M4 & 29 & 0.048 & 0.018 & 8.73 & 3.26 & 0.0015 & 100 \\
\hline M5 & 22 & 0.097 & 0.011 & 40.82 & 5.01 & 0.004 & 100 \\
\hline M6 & 23 & 0.056 & 0.020 & 28.42 & 10.60 & 0.002 & 96.4 \\
\hline M7 & 22 & 0.137 & 0.016 & 65.04 & 8.78 & 0.0051 & 93.5 \\
\hline
\end{tabular}

Table 7.7.2 Response of female subjects in the younger age group to the supplement consumption considering the baseline and final MPOD values from table 7.6.2. The rate of change involves all the interim measurements done during the 6 month study. 


\begin{tabular}{|c|c|c|c|c|c|c|c|}
\hline \multirow[t]{2}{*}{$\begin{array}{l}\text { Subje } \\
\text { ct } \\
\text { No. }\end{array}$} & \multirow[t]{2}{*}{$\begin{array}{l}\text { Age } \\
\text { (yrs.) }\end{array}$} & \multicolumn{2}{|c|}{$\begin{array}{l}\text { Changes in } \\
\text { MPOD and } \\
\text { SEM(d.u) }\end{array}$} & \multicolumn{2}{|c|}{$\begin{array}{l}\text { \% Change in } \\
\text { MPOD and SEM }\end{array}$} & \multirow{2}{*}{$\begin{array}{l}\text { Rate of } \\
\text { change of } \\
\text { MPOD } \\
\text { (d.u.)/wee } \\
\text { k }\end{array}$} & \multirow[t]{2}{*}{$\begin{array}{l}\text { Percentage } \\
\text { Compliance }\end{array}$} \\
\hline & & $\begin{array}{l}\text { MPOD } \\
\text { change }\end{array}$ & $\begin{array}{l}\text { SEM } \\
\text { change }\end{array}$ & $\begin{array}{l}\text { \% change } \\
\text { in MPOD }\end{array}$ & $\begin{array}{l}\text { SEM in \% } \\
\text { change }\end{array}$ & & \\
\hline M13 & 51 & 0.050 & 0.013 & 6.64 & 1.67 & 0.002 & 91 \\
\hline M15 & 61 & -0.003 & 0.016 & -0.54 & -2.69 & -0.002 & 99.4 \\
\hline L15 & 70 & -0.010 & 0.010 & -1.34 & -1.28 & 0.002 & 100 \\
\hline L16 & 61 & 0.031 & 0.012 & 7.30 & 2.71 & 0.002 & 94.7 \\
\hline L17 & 52 & 0.026 & 0.027 & 4.73 & 4.83 & 0.001 & 99.4 \\
\hline L18 & 51 & 0.068 & 0.013 & 13.54 & 2.66 & 0.002 & 100 \\
\hline L19 & 53 & 0.059 & 0.015 & 9.79 & 2.45 & 0.003 & 99.4 \\
\hline L20 & 52 & 0.037 & 0.017 & 5.53 & 2.46 & 0.002 & 83.4 \\
\hline $\mathrm{L} 22$ & 71 & 0.102 & 0.023 & 20.84 & 4.68 & 0.004 & 98.2 \\
\hline
\end{tabular}

Table7.7.3 Response of the male subjects in the older age group to the supplement consumption considering the baseline and the final MPOD values from table 7.6.3. The rate of change involves all the interim measurements done during the 6 month study.

\begin{tabular}{|l|l|l|l|l|l|l|l|}
\hline $\begin{array}{l}\text { Subject } \\
\text { No. }\end{array}$ & $\begin{array}{l}\text { Age } \\
\text { (yrs.) }\end{array}$ & \multicolumn{2}{l|}{$\begin{array}{l}\text { Changes in } \\
\text { MPOD } \\
\text { and SEM(d.u) }\end{array}$} & \multicolumn{2}{l|}{$\begin{array}{l}\text { \% Change in } \\
\text { MPOD and SEM }\end{array}$} & $\begin{array}{l}\text { Rate of } \\
\text { Change } \\
\text { (d.u.)/week }\end{array}$ & $\begin{array}{l}\text { Percentage } \\
\text { Compliance }\end{array}$ \\
\cline { 2 - 6 } & & $\begin{array}{l}\text { MPOD } \\
\text { change }\end{array}$ & $\begin{array}{l}\text { SEM } \\
\text { change }\end{array}$ & $\begin{array}{l}\text { \% change } \\
\text { in MPOD }\end{array}$ & $\begin{array}{l}\text { SEM in \% } \\
\text { change }\end{array}$ & & \\
\hline M14 & 55 & 0.032 & 0.015 & 7.35 & 3.44 & 0.001 & 100 \\
\hline L13 & 63 & 0.008 & 0.030 & 2.62 & 10.08 & 0.0005 & 94.7 \\
\hline L21 & 51 & 0.066 & 0.023 & 29.66 & 10.37 & 0.003 & 97.6 \\
\hline L23 & 50 & -0.042 & 0.016 & -13.37 & -5.02 & -0.002 & 93.5 \\
\hline L24 & 53 & 0.038 & 0.020 & 8.57 & 4.55 & 0.001 & 96.4 \\
\hline
\end{tabular}

Table7.7.4 Response of the female subjects in the older age group to the supplement consumption considering the baseline and the final MPOD values from table 7.6.4. The rate of change involves all the interim measurements done during the 6 month study. 


\begin{tabular}{|c|c|c|c|c|c|c|c|c|}
\hline \multirow[t]{3}{*}{ Supplement } & \multicolumn{4}{|c|}{$\begin{array}{c}\text { Average change in MPOD and } \\
\text { SEM in (d.u.) }\end{array}$} & \multicolumn{2}{|c|}{$\begin{array}{l}\text { Average \% } \\
\text { change in } \\
\text { Younger group }\end{array}$} & \multicolumn{2}{|c|}{$\begin{array}{l}\text { Average \% change in } \\
\text { Older group }\end{array}$} \\
\hline & \multicolumn{2}{|c|}{ MPOD } & \multicolumn{2}{|l|}{ SEM } & \multirow[t]{2}{*}{ MPOD } & \multirow[t]{2}{*}{ SEM } & \multirow[t]{2}{*}{ MPOD } & \multirow[t]{2}{*}{ SEM } \\
\hline & Young & Old & Young & Old & & & & \\
\hline Lutein & 0.053 & 0.042 & 0.017 & 0.019 & 14.94 & 5.37 & 7.98 & 3.49 \\
\hline "Micro" & 0.070 & 0.026 & 0.017 & 0.014 & 26.74 & 3.99 & 4.48 & 0.81 \\
\hline
\end{tabular}

Table7.7.5 Overall response to the supplementation across the age groups.

\section{Section 7.8}

\section{Discussion}

A change in the level of MP was observed for all the subjects who participated in the study. To test if such changes observed were significant or not, a two tailed Student's t-test was conducted. A 0.05 confidence level was used to infer if changes in the MPOD values were significant or not. The subjects who were found to have significant change in MPOD taking the lutein supplement are given in the table 7.8.1 below. 


\begin{tabular}{|l|l|l|l|c|}
\hline Subject Code & Supplement & Gender & $\begin{array}{l}\text { Age } \\
\text { Group }\end{array}$ & Increase/Decrease \\
\hline L1 & Lutein & Male & Young & Increase \\
\hline L2 & Lutein & Female & Young & Decrease \\
\hline L3 & Lutein & Male & Young & Increase \\
\hline L4 & Lutein & Male & Young & Increase \\
\hline L5 & Lutein & Male & Young & Increase \\
\hline L6 & Lutein & Female & Young & Increase \\
\hline L8 & Lutein & Female & Young & Increase \\
\hline L9 & Lutein & Male & Young & Increase \\
\hline L10 & Lutein & Male & Young & Increase \\
\hline L12 & Lutein & Female & Young & Increase \\
\hline L13 & Lutein & Female & Old & Increase \\
\hline L18 & Lutein & Male & Old & Increase \\
\hline L19 & Lutein & Male & Old & Increase \\
\hline L21 & Lutein & Female & Old & Increase \\
\hline L22 & Lutein & Male & Old & Increase \\
\hline L23 & Lutein & Female & Old & Decrease \\
\hline L24 & Lutein & Female & Old & Increase \\
\hline
\end{tabular}

Table 7.8.1 Subjects on the Lutein supplement with significant change in their MPOD levels at the end of the study.

Similarly subjects who were on the "Micro" supplements and had shown a significant change in their MPOD levels are given in table 7.8.2.

\begin{tabular}{|c|c|c|c|c|}
\hline Subject Code & Supplement & Gender & $\begin{array}{c}\text { Age } \\
\text { Group }\end{array}$ & Increase/Decrease \\
\hline M1 & Micro & Female & Young & Increase \\
\hline M3 & Micro & Male & Young & Increase \\
\hline M4 & Micro & Female & Young & Increase \\
\hline M5 & Micro & Female & Young & Increase \\
\hline M6 & Micro & Female & Young & Increase \\
\hline M7 & Micro & Female & Young & Increase \\
\hline M13 & Micro & Male & Old & Increase \\
\hline M14 & Micro & Female & Old & Increase \\
\hline
\end{tabular}

Table 7.8.2 Subjects on the "Micro" supplement with significant change in their MPOD levels at the end of the study.

The subjects that did not show significant changes in their MPOD at the 0.05

confidence level consuming either of the supplements are given in table 7.8.3. 


\begin{tabular}{|l|l|l|l|l|}
\hline Subject Code & Supplement & Gender & Age Group & Increase/Decrease \\
\hline L11 & Lutein & Female & Young & Increase \\
\hline L15 & Lutein & Male & Old & Decrease \\
\hline L16 & Lutein & Male & Old & Increase \\
\hline L17 & Lutein & Male & Old & Increase \\
\hline L20 & Lutein & Male & Old & Increase \\
\hline M2 & Micro & Female & Young & Decrease \\
\hline M15 & Micro & Male & Old & Decrease \\
\hline
\end{tabular}

Table 7.8.3 List of subjects whose MPOD levels was not changed significantly at the end of the study.

On inspection of tables 7.8.1 and 7.8.2, we observe that more individuals in the younger age group have shown significant changes in their MPOD levels than individuals in the older age group.

The slope of the regression line obtained for the plot of average weighted MPOD values with respect to the week number was analyzed by performing a t-test to determine whether it was significantly different from zero. A 0.05 confidence level was set to test for significance. The subjects on the lutein supplement grouped in table 7.8.1 that had shown a significant difference between their MPOD values at the start and end of the study were tested to determine if their rate of change of MPOD was significantly different from zero. These are listed in table 7.8.4. In table 7.8.5 we listed the subjects from table 7.8.2 who had shown a significant change in their MPOD and were on the "Micro" supplement. 


\begin{tabular}{|c|c|c|c|}
\hline Subject & Supplement & Gender & Age Group \\
\hline L2 & Lutein & Female & Young \\
\hline L3 & Lutein & Male & Young \\
\hline L4 & Lutein & Male & Young \\
\hline L5 & Lutein & Male & Young \\
\hline L21 & Lutein & Female & Old \\
\hline L22 & Lutein & Male & Old \\
\hline
\end{tabular}

Table 7.8.4 Subjects on Lutein supplement who had shown an overall significant change and a significant rate of change in their MPODs

\begin{tabular}{|c|c|c|c|}
\hline Subject & Supplement & Gender & Age Group \\
\hline M1 & Micro & Female & Young \\
\hline M5 & Micro & Female & Young \\
\hline M6 & Micro & Female & Young \\
\hline M7 & Micro & Female & Young \\
\hline
\end{tabular}

Table 7.8.5 Subjects on the "Micro" supplement who had shown an overall significant change and a significant rate of change in their MPODs

We observe from table 7.8.4 that for the lutein supplement, more subjects in the younger age group had shown a significant rate of change in their MPOD values. The significant rate of change amongst younger subjects is only observed in table 7.8.5 with the “micro” supplement.

Referring to table 7.7.5 we observe that average percentage increase in MPOD for subjects on lutein has been almost the double the value for the younger individuals compared to that observed among the older individuals. For the "Micro" supplement, the overall percentage change in the MPOD values is seen 
to be even more pronounced compared to what we observe in the lutein group. A factor that might stand out as pertinent in observing such a trend might be based on the baseline or the pre-supplemental values of MPOD measured. This proposition was pointed out in Bone et al. ${ }^{17}$ The baseline values in the older age group are observed to be comparatively higher than the baseline values in the younger age group. If the value of saturation of lutein and zeaxanthin in the retina had already been reached in the individuals as indicated by a higher baseline value, a slower rate of change in the MPOD values can be expected due to supplementation. Hence apparently the younger group had a better response to the supplementation than the older group with both formulations. Further, the average baseline values of the MPOD of the younger subjects are lower in the "Micro" group than the lutein group, which again might be an indicator that higher levels of rise might be expected amongst the younger subjects in the "Micro" group than the younger subjects in the lutein group as seen in table 7.7.5.

In table 7.8.4 we observe that both males and females on the lutein supplement have shown significant rates of changes in their MPOD. Following table 7.8.5, we observe that only females in the younger age group taking the "Micro" supplement have shown a significant rate of change in their MPOD. However, due to the limited amount of data, we cannot pursue a gender-based analysis of the responses in each age group.

We have observed that out of 32 subjects who participated, 25 showed a significant overall change in their MPOD levels. Out of the 25 subjects, 10 
individuals have clearly shown a significant rate of change in their MPOD level during the study. As changes in MPOD levels could be monitored and measured with Mapcatsf, it could be considered a choice of clinical practitioners for use in similar such supplement studies. 


\section{Chapter 8}

\section{Protective Role of Lutein found in the Lens of the Human Eye.}

\section{Section 8.1}

\section{Introduction:}

The lens in the eye is crystalline in nature and composed of water soluble proteins $^{118}$. The components of the lens undergo structural changes leading to changes in opacity levels which at advanced stages renders the lens, or the pertinent region of the lens, cataractous ${ }^{76}$. Cataract is one of the leading causes of vision impairment in the world especially amongst the elderly. ${ }^{77}$

Refraction $^{119}$ and transmittance ${ }^{120}$ of light by the lens are the two major factors affecting the quality of the image formed on the retina. Therefore for maintenance of good visual performance, transparency of the lens is of major importance.

The overall optical density of the lens changes with age ${ }^{47}$,with the nuclear ${ }^{121}$ and the cortical $^{121}$ regions being the primary areas showing age-related opacity changes. However,opacity developed in the posterior subcapsular region is not age-related $^{79}$ but due to reasons such as steroid intake ${ }^{122}$, diabetes ${ }^{123}$ and exposure to ionizing radiation ${ }^{124}$.

Though there are various factors associated with the three types of cataracts mentioned in chapter 6 , the lens being subjected to oxidative stress is a common factor responsible for the occurrence of cortical and nuclear cataracts. ${ }^{125}$ 
Oxidative stresses are due to UV light exposure, ${ }^{126}$ oxidants in the ocular fluids, ${ }^{127}$ and endogenous oxidants which are produced in the lens. ${ }^{128}$ Traces of lutein and zeaxanthin have been reported to be found in the human lens. ${ }^{129,64} \mathrm{~A}$ protective role of the carotenoids lutein and zeaxanthin from oxidative stress is also believed to be associated with them acting in protective capacity for lipids found in the lens which are susceptible to attack by free oxygen radicals. ${ }^{130}$

As stated in chapter 1, lutein and zeaxanthin have their major presence as the constituents of MP in the primate retina. Levels of lutein and zeaxanthin in the blood are indicative of the MPOD. ${ }^{131}$ The lens indirectly derives its nutrients from blood $^{132}$ via the aqueous humor. Therefore MPOD might be expected to be correlated with levels of lutein and zeaxanthin in the lens.

Our prime goal in this study was to investigate the existence of a relationship as proposed in Hammond et al 1997, where the variation of the lens optical density with MPOD was studied and a negative trend was reported between the two quantities. Such a study is of great interest since lutein and zeaxanthin are the only two carotenoids that have been identified in the lens, and a diet rich in lutein and zeaxanthin has been shown to be a factor in preventing development of cataracts. $^{133}$

Our tests were conducted using Mapcatsf for determining both the MPOD and the lens optical density. 


\section{Section 8.2}

\section{Methods:}

\section{Subject Recruitment:}

A total of 89 subjects were recruited in the age range of 18 to 80 years with an average age \pm SD of $34 \pm 15$ years. The subjects recruited were in good visual health, and were non-smokers. All the subjects signed an informed consent form approved by FIU-IRB prior to testing. Since there have been reports of high levels of concordance of both the MPOD ${ }^{134}$ and the lens densities ${ }^{56}$ between both the eyes, only the right eye was tested.

\section{Section 8.3}

\section{MPOD and LOD measurement:}

A detailed description of Mapcatsf and the testing procedure for both the MPOD and LOD is included in chapter 2.

\section{Section 8.4}

\section{Results}

The total recruitment of subjects was divided into three age groups: young (18 to 30 years), middle aged (30 to 50 years) and old (50 to 80 years). The data for each age group were further classified based on gender as given in table 8.1. In tables 8.2 and 8.3 the average MPOD and the lens density values were calculated separately for males and females in each age group. 


\begin{tabular}{|c|c|c|c|c|c|c|c|c|c|}
\hline \multirow{2}{*}{ Gender } & \multicolumn{3}{|c|}{ Age 18 to 30 years } & \multicolumn{3}{c|}{ Age 30 to 50 years } & \multicolumn{3}{c|}{ Age 50 to 80 years } \\
\cline { 2 - 10 } & No. & $\begin{array}{c}\text { Mean } \\
\text { Age(yrs.) }\end{array}$ & $\begin{array}{c}\text { S.D. } \\
\text { (yrs.) }\end{array}$ & No. & $\begin{array}{c}\text { Mean Age } \\
\text { (yrs.) }\end{array}$ & $\begin{array}{c}\text { S.D. } \\
\text { (yrs.) }\end{array}$ & No. & $\begin{array}{c}\text { Mean } \\
\text { Age(yrs.) }\end{array}$ & $\begin{array}{c}\text { S.D. } \\
\text { (yrs.) }\end{array}$ \\
\hline Males & 27 & 23.5 & 3.06 & 10 & 35.5 & 4.30 & 13 & 58 & 6.37 \\
\hline Females & 23 & 22.86 & 2.73 & 5 & 37 & 5.52 & 11 & 58.6 & 7.18 \\
\hline
\end{tabular}

Table 8.1 Age- and gender-based classification of the participating subjects.

\begin{tabular}{|l|l|l|l|l|l|l|l|l|l|}
\hline Gender & \multicolumn{3}{|l|}{ Age 18 to 30 years } & \multicolumn{3}{|l|}{ Age 30 to 50 years } & \multicolumn{3}{l|}{ Age 50 to 80 years } \\
\hline & $\begin{array}{l}\text { Mean } \\
\text { test } \\
\text { values }\end{array}$ & $\begin{array}{l}\text { Mean } \\
\text { retest } \\
\text { values }\end{array}$ & $\begin{array}{l}\text { Mean } \\
\text { test, } \\
\text { retest }\end{array}$ & $\begin{array}{l}\text { Mean } \\
\text { test } \\
\text { values }\end{array}$ & $\begin{array}{l}\text { Mean } \\
\text { retest } \\
\text { values }\end{array}$ & $\begin{array}{l}\text { Mean } \\
\text { test, } \\
\text { retest }\end{array}$ & $\begin{array}{l}\text { Mean } \\
\text { test } \\
\text { values }\end{array}$ & $\begin{array}{l}\text { Mean } \\
\text { retest } \\
\text { values }\end{array}$ & $\begin{array}{l}\text { Mean } \\
\text { test, } \\
\text { retest }\end{array}$ \\
\hline Male & 0.445 & 0.432 & 0.438 & 0.471 & 0.468 & 0.469 & 0.556 & 0.564 & 0.560 \\
\hline Female & 0.406 & 0.407 & 0.407 & 0.566 & 0.556 & 0.561 & 0.438 & 0.429 & 0.433 \\
\hline
\end{tabular}

Table 8.2 Average of MPOD obtained from test retest for the subjects. 


\begin{tabular}{|c|c|c|c|c|c|c|c|c|c|}
\hline \multirow{2}{*}{ Gender } & \multicolumn{3}{|c|}{ Age 18 to 30 years } & \multicolumn{3}{c|}{ Age 30 to 50 years } & \multicolumn{3}{c|}{ Age 50 to 80 years } \\
\cline { 2 - 9 } & $\begin{array}{c}\text { Mean } \\
\text { test } \\
\text { values }\end{array}$ & $\begin{array}{c}\text { Mean } \\
\text { retest } \\
\text { values }\end{array}$ & $\begin{array}{c}\text { Mean } \\
\text { test, } \\
\text { retest }\end{array}$ & $\begin{array}{c}\text { Mean } \\
\text { test }\end{array}$ & $\begin{array}{c}\text { Mean } \\
\text { retest } \\
\text { values }\end{array}$ & $\begin{array}{c}\text { Mean } \\
\text { test, } \\
\text { retest }\end{array}$ & $\begin{array}{c}\text { Mean } \\
\text { test }\end{array}$ & $\begin{array}{c}\text { Mean } \\
\text { retest }\end{array}$ & $\begin{array}{c}\text { Mean } \\
\text { test, } \\
\text { retest }\end{array}$ \\
\hline Male & 0.549 & 0.562 & 0.555 & 0.620 & 0.642 & 0.631 & 0.891 & 0.900 & 0.895 \\
\hline Female & 0.498 & 0.513 & 0.505 & 0.635 & 0.659 & 0.647 & 0.936 & 0.951 & 0.944 \\
\hline
\end{tabular}

Table 8.3 Average of LOD obtained from test retest for the subjects.

A scatter plot shown in Fig. 8.1 followed by a regression analysis was pursued in order to investigate the overall trend of the variation of LOD with MPOD for all the age groups combined.

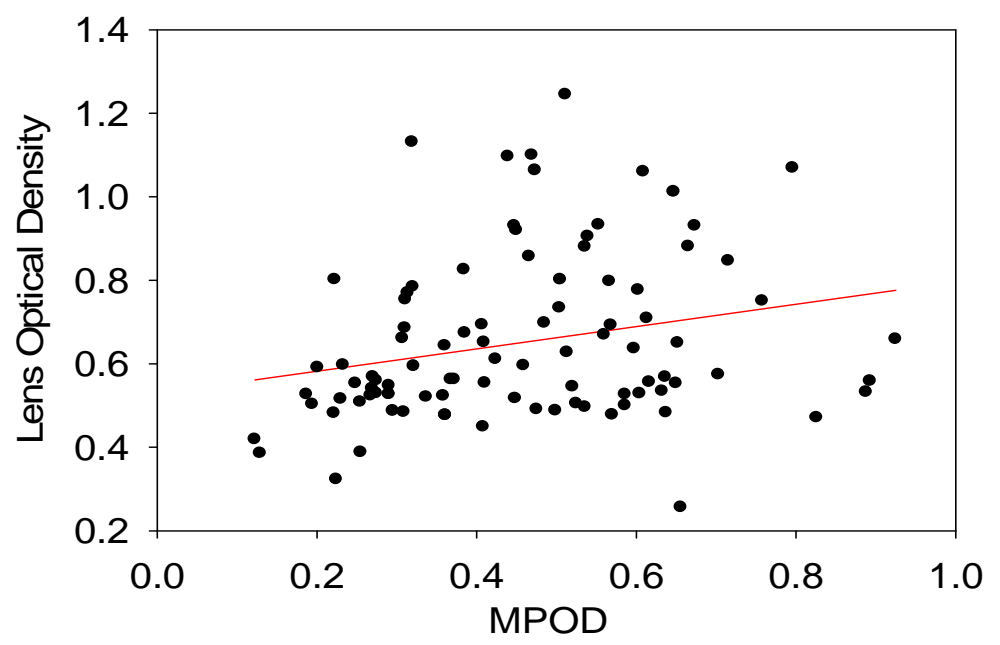

Fig. 8.1 Scatter plot of LOD with MPOD for all subjects. The regression line shown in red is given by the Eq.: $y=0.528+0.267 x$, with a $p<0.0175$. 
On regression analysis of the scatter plot in Fig. 8.1, we obtained a positive slope. The slope and the intercept were 0.267 and 0.528 respectively. The $r^{2}$ value obtained was 0.0598 with $\mathrm{p}<0.0175$, indicating a very low level of correlation between LOD and MPOD.

To observe the effect in each age group of the variation of LOD with MPOD, we plotted the data for each age group as shown in Fig. 8.2.

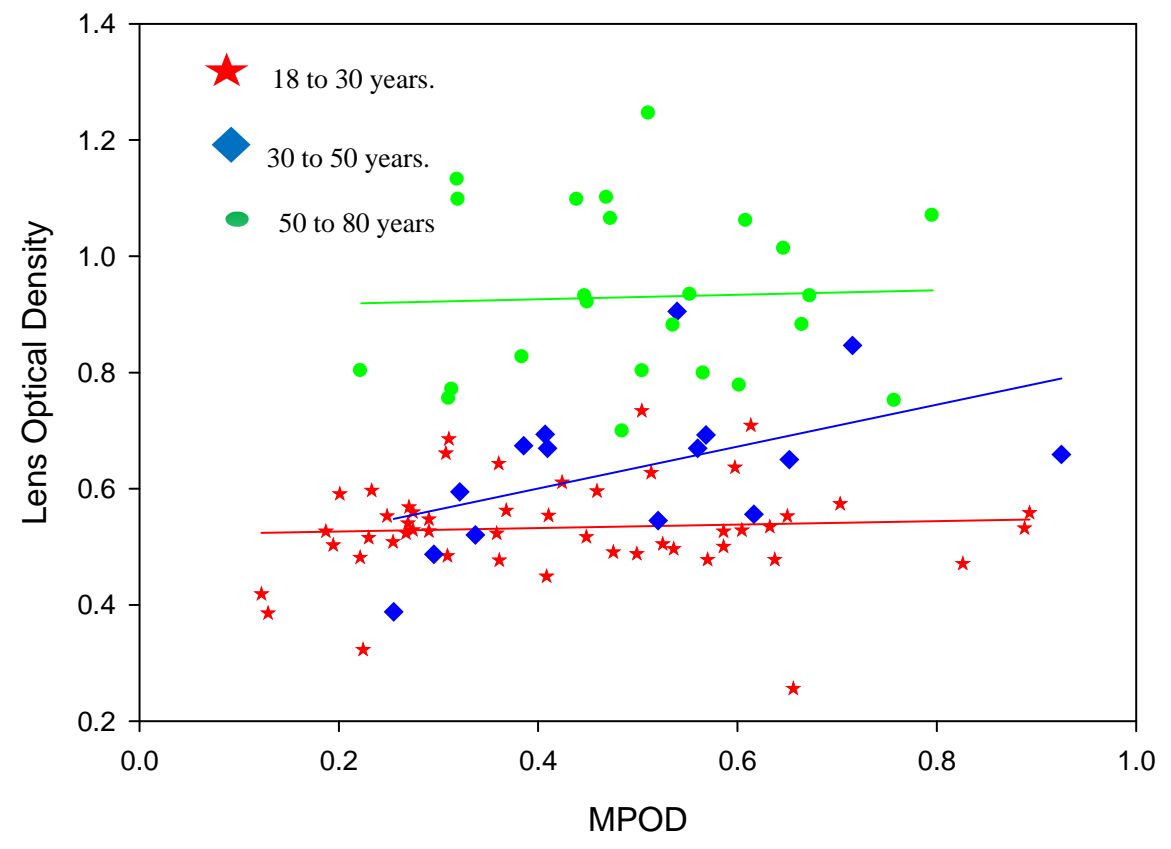

Fig. 8.2 Regression lines for the scatter plots of LOD with MPOD for the three age groups. For the group of subjects between 18 and 30 years the Eq. of the line was $y=0.514+0.056 \mathrm{x}$ (shown in red), $\mathrm{p}$ value $=0.3863$; For the group of subjects between 30 and 50 years the Eq. of the regression line was $y=0.469+0.341 x$ (shown in blue), p value $=0.0867$; and the Eq. of the regression line for the group of subjects between 50 and 80 years was $y=0.866+0.107 x$ (shown in green), pvalue $=0.625$ 
Further, we investigated whether there was a difference between males and females in the separate age groups regarding the relation of MPOD to LOD . The results are shown in Figs 8.3 and 8.4.

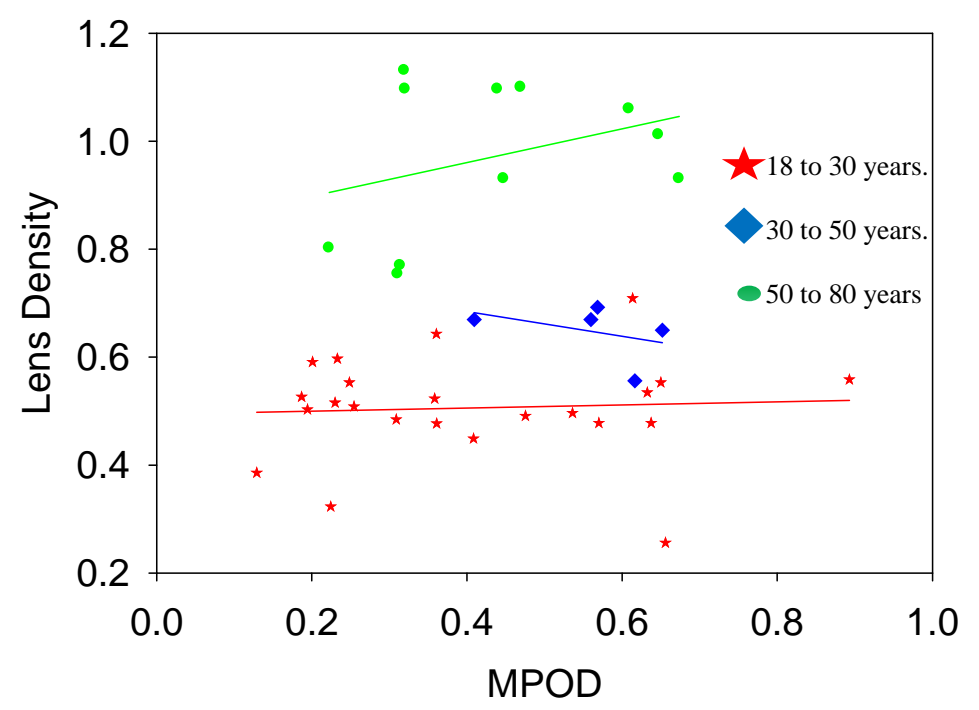

Fig. 8.3 Regression lines for scatter plots of LOD with MPOD for females in the three age groups. For the age group of females between 18 and 30 years the regression line has an Eq.: $y=0.494+0.028 x$ (shown in red), $p$ value $=0.784$.For the age group of females between 30 and 50 years the regression line has an Eq. $y=0.775-0.228 x$ (shown in blue), $p$-value $=0.507$. For females in the age group of 50 to 80 years the regression line: $\mathrm{y}=0.836+0.312 \mathrm{x}$ (shown in green), $\mathrm{p}$ value $=0.312$. 


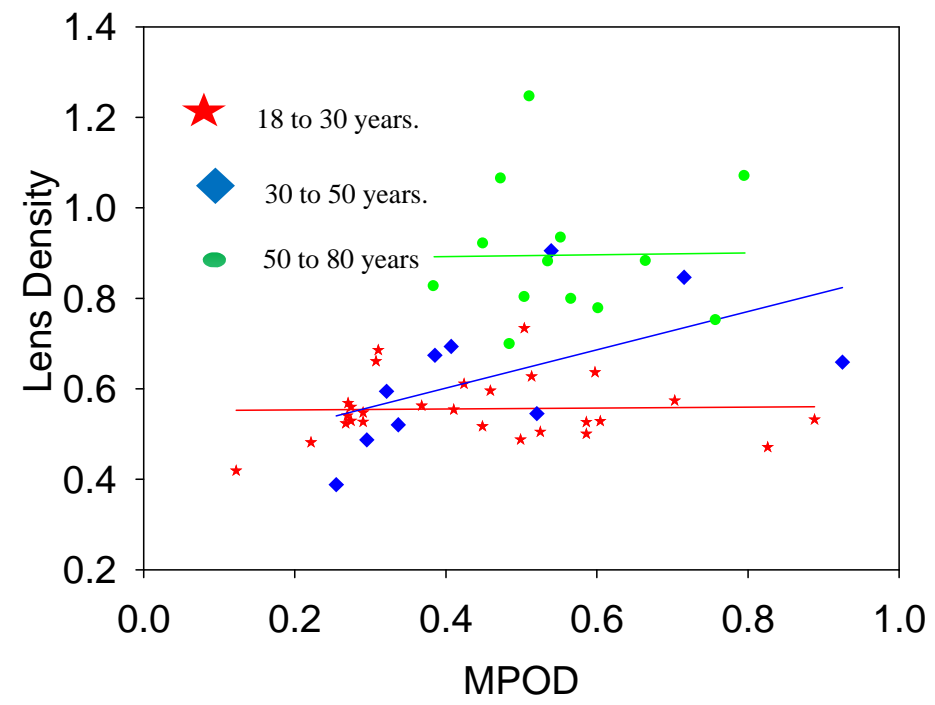

Fig. 8.4 Regression lines for scatter plots of LOD with MPOD for males in the three age groups. For the age group of males between 18 and 30 years the regression line has an Eq.: $y=0.551+0.009 x$ (shown in red), p-value $=0.895$. For the age group of males between 30 and 50 years the regression line has an Eq. $y=0.432+0.423 x$ (shown in blue), $p$-value $=0.0914$. For males in the age group of 50 to 80 years the regression line: $y=0.885+0.019 x$ (shown in green), $p$ value $=0.962$

\section{Section 8.5}

\section{Discussion:}

The regression line in Fig. 8.1 has a positive slope. Such a trend was also reported by Hammond et al. $1997{ }^{18}$ When analysis was pursued on the scatter plots for each age group separately (shown in Fig. 8.2), the regression lines had positive slopes, albeit very small except for the 30 to 50 age group. Such a trend contradicts the relationship indicated in Fig. 2 of Hammond et al 1997 for the age groups considered "middle aged” and "oldest” where they had reported a decline in LOD with the increase in MPOD of the subjects. However, the upper and lower 
ages of the subjects in the middle and the older age groups in our study were different than those in Hammond et al. 1997. In the study conducted by Hammond et al, their middle age group involved individuals between 48 and 66 years of age, and the older age group involved subjects between 67 and 82 years of age. In our study the middle age group consisted of individuals between 30 and 50 years and the older age group comprised of individuals between 50 and 80 years. The variation in the upper and lower age limits in both the studies prevents a direct comparison of the data obtained from the two studies. As LOD increases with the subject's age, it might be that older subjects were more conscious about their diet, resulting in the consumption of carotenoid-rich foods such as spinach ${ }^{135}$ and kale ${ }^{136}$ and higher levels of lutein and zeaxanthin in the blood serum and the fovea. Based on this hypothesis, a positive correlation between lens density (a surrogate of biological age) and MPOD (a surrogate of lens carotenoids) might be expected. However such a proposition cannot be well supported without conducting an experiment on subjects with their dietary habits taken into consideration. Similarly, aside from dietary habits, lifestyle, especially a measure of the amount of exposure to UV radiation of the subjects, should also be considered. ${ }^{137,83}$ Also an important step towards further improving LOD measurement would be not to involve photopic responses whereby measurements could be affected by the variation of the $\mathrm{L}$ and $\mathrm{M}$ cones in the retina. ${ }^{56}$

From an overall standpoint, both the LOD and MPOD measured depend on various factors such as dietary habits and UV exposure. Therefore when subjects 
are recruited, care should be taken to include these factors for the purpose of having better understanding of the overall statistical outcomes. 


\section{Chapter 9}

\section{Role of Involuntary Eye Movements in Designing an Optimum Gaze Facilitating Stimulus}

\section{Section 9.1}

\section{Introduction}

Though the MP is normally considered to have its peak concentration in the center of the fovea, ${ }^{2}$ there are published research findings that reveal a variability in the concentration around the region of the fovea for some individuals. ${ }^{138,139,140}$ Such findings have led researchers to ask the question: when MPOD is measured by HFP by imaging a stimulus around the foveal region of the retina, how the measured value can be interpreted taking into account the variability of an individual's MP distribution? Though as a naïve approach one can consider the value obtained is an overall average of the distribution, there have been results from studies that have been accepted which claim that MPOD measured by HFP is actually the value at a location in the retina associated with the edge of the image formed by the foveal stimulus. ${ }^{66,33,141}$ However Bone, Landrum, and Gibert in $2004^{48}$ published an article which contradicted this hypothesis. In their study they modified an HFP instrument by using annular stimuli of different diameters up to $1.5^{\circ}$ (the diameter of the foveal stimulus originally used). Such an alteration helped them determine the average MPOD at various eccentricities. Similar testing and analysis was conducted by comparing a $1^{\circ}$ diameter foveal stimulus 
with an annular stimulus of the same diameter. For both the $1^{\circ}$ and $1.5^{\circ}$ stimuli, the average MPOD value was less than that of the corresponding annular stimulus. Such observations led them to interpret the proposition of an edge effect in MPOD measurement to be an artifact of fixational eye movements.

These eye movements can be classified into three types which are tremor, microsaccades and drift. ${ }^{49}$ Microsaccades are generally associated with peripheral vision. Drift movements are believed to have a compensatory role in maintenance of gaze ${ }^{13}$ and therefore are associated with foveal vision ${ }^{142}$ and have amplitudes of the order of $0.36^{\circ} .{ }^{50}$ Such orders of magnitude of the drift movements can lead to alteration of the area of the retina sampled during MPOD measurements. If the flicker null/minimum settings are made during the course of such movements, it is quite likely that the measured value of MPOD might be closer to the value at the edge of the stimulus.

An experiment was therefore conducted to test whether the cross-hairs used with the Mapcatsf stimuli represented the optimum design for accurate fixation, or whether alternative designs might lead to improved fixation.

\section{Section 9.2}

\section{Experimental Setup}

Three types of central gaze facilitating foveal stimuli were designed as shown in Fig. 9.1. 

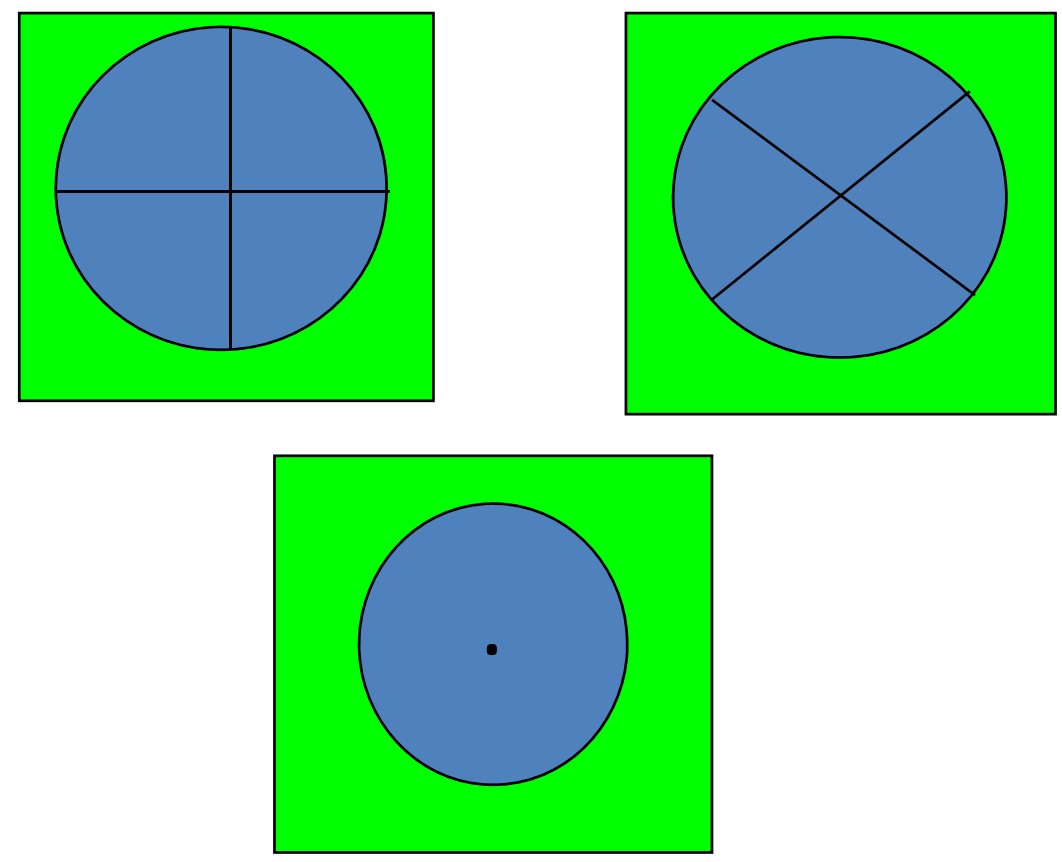

Fig.9.1The types of configurations of foveal stimuli tested for facilitating minimum fixational eye movements.

The experimental setup consisted of a projector, a screen, and a chair as shown in Fig 9.2. The chair had a head mount and a chin rest attached. It was used to ensure that the subject was in a head-fixed position at a fixed distance from the screen. This setup was used so that the projected stimulus subtended exactly $1.5^{\circ}$ diameter for the participating subject, the same as the size of the foveal stimulus in Mapcatsf $^{\mathrm{TM}}$. 


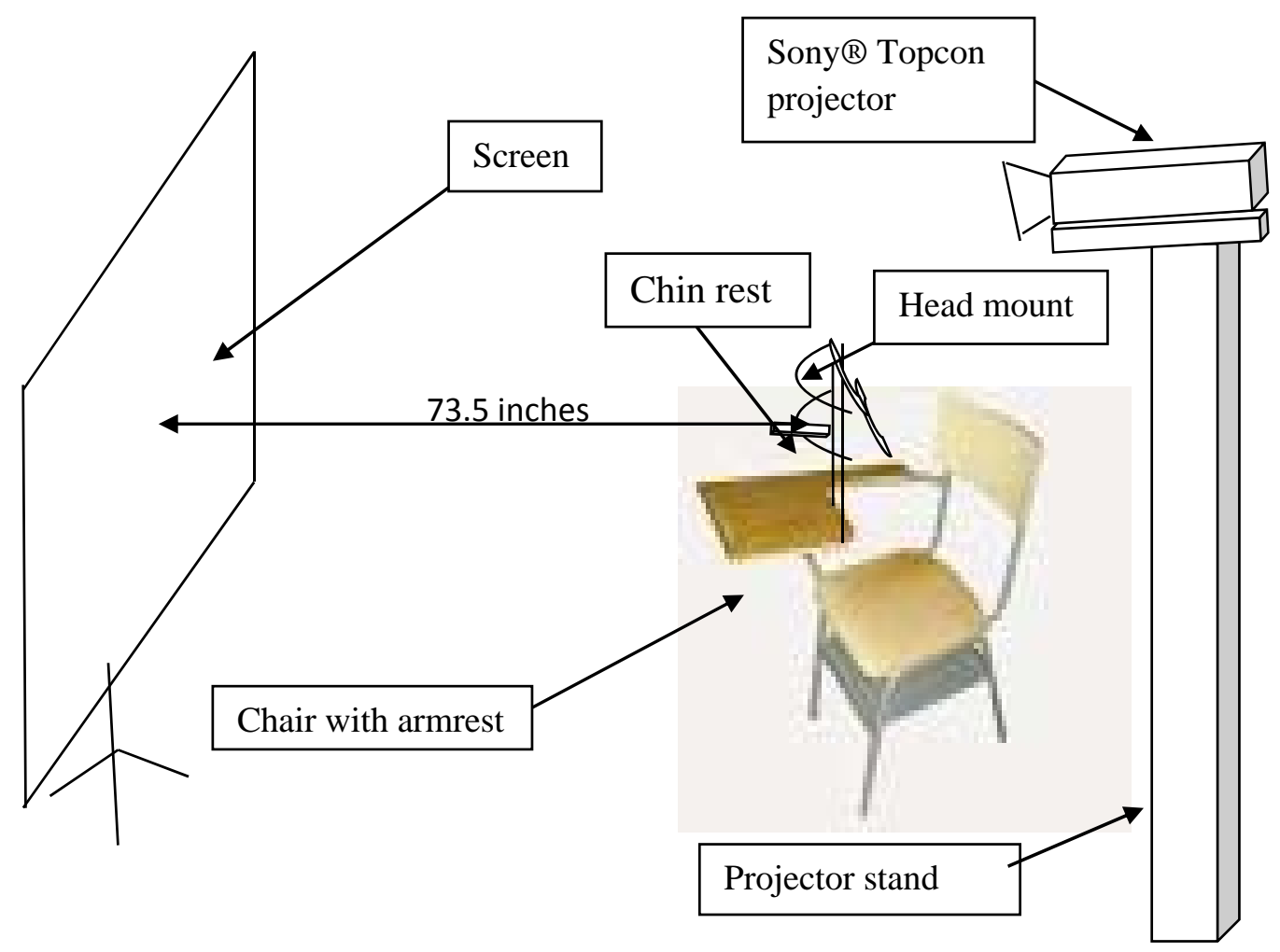

Fig. 9. 2 The screen and projector setup

To record the eye movements associated with fixation, the primary instrument used for the study was a commercial software-controlled eye tracker, "Viewpoint PC-60”, manufactured by Arrington Research Systems, Scottsdale Arizona USA. The eye tracker was designed for tracking monocular gaze only for the left eye. It comprised a CCD camera of 360 by 240 pixels resolution that captured the scene at 30 frames per second. An infrared camera tracked the center of the pupil which was illuminated by an infrared LED while the subject gazed at the stimulus. The scene camera, eye tracking infrared camera and infrared LED were assembled on an eyewear frame which could be worn by the subject. The eyewear setup for the eye tracker is shown in Fig.3. 


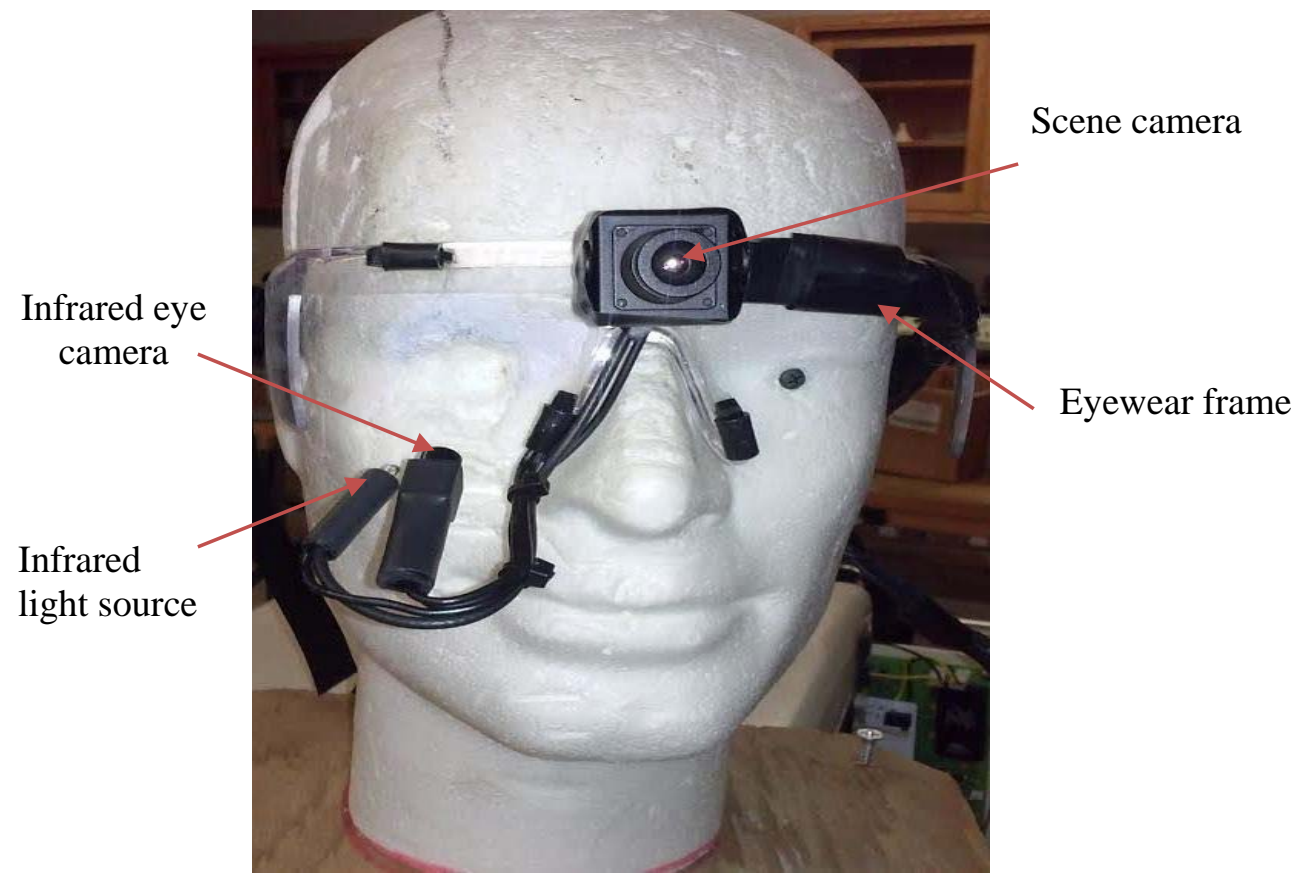

Fig. 9.3 The hardware components of the eyetracker

The window corresponding to the tracking software is shown in the Fig 9.4. An Excel file recorded the $\mathrm{x}$ and $\mathrm{y}$ coordinates with respect to the scene as captured in an “.avi” file. 


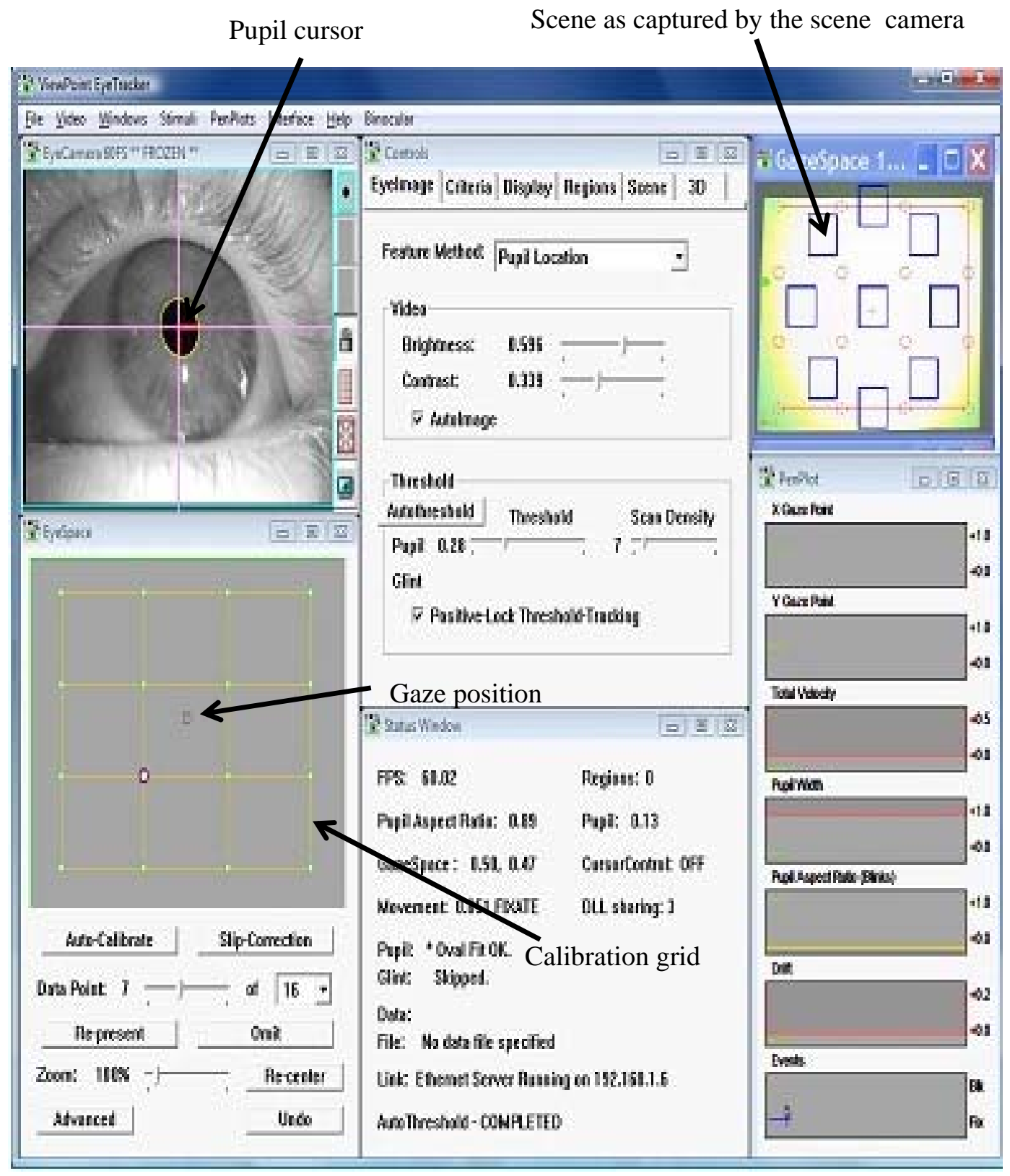

Fig. 9.4 The controlling window for the eyetracker. ${ }^{143}$ 


\section{Section 9.3}

\section{Experiment}

Ten subjects with no vision anomalies were recruited and signed an informed consent approved by the FIU-IRB. Each subject was required to participate three times aside from one subject who was only able to participate twice. Prior to the start of the experiment, the subjects had their right, non-tracking eye occluded with an eyepatch. This was done for two reasons. The primary reason was that Mapcatsf measures MPOD of one eye at a time and the other reason was, according to feedback from subjects, while getting tested on Mapcatsf, the availability of the non-testing eye for vision made gaze fixation of the testing eye difficult. The subject was then guided to put on the eyetracker as shown in Fig 9.3. Once a proper alignment of the eyewear was obtained, the pupil cursor was locked within the region of interest to avoid unnecessary shifts.

With the eye camera being properly aligned to ensure optimum tracking, the subjects were seated with their chin on the chin rest and their head restrained with a forehead rest. The operator subsequently adjusted the alignment of the scene camera on the eyewear to fit the entire screen in the field of the scene camera as shown in the Gaze Space window of Fig.9.4.. A head-fixed calibration was then conducted so that, when mapped, the gaze coordinates would be well correlated to the movie frames captured by the scene camera.

The calibration was carried out by the operator by pointing a laser pointer at the desired point on the screen as monitored on the Gaze Space of the tracking 
software. Once the subject's gaze was precisely located at the desired point on the scene, the calibration point was recorded on the Eye Space of the window of the tracking software by the operator. This was repeated 15 more times until the points were well spread and when joined by straight lines appeared approximately as a rectangular grid. A well calibrated grid is shown in Fig. 9.4. To correct for any consistent offset between the line of sight of the scene camera and the eye, a slip correction was done. The slip correction was carried out by selecting a point in the Eye Space window and the operator directing the subject to look at the laser pointer pointed at the corresponding point on the screen. The operator subsequently clicked the “Slip-Correction” button on the Eye-Space.

Once a proper alignment, calibration and slip correction were obtained, the subject was presented sequentially with the three stimuli shown in Fig 9.1. For each presentation of a stimulus, the subject was directed to fixate his/her gaze at the center of the stimulus for 60 seconds duration. During these 60 seconds, both the scene and the corresponding gaze parameters, including the $\mathrm{x}$ and $\mathrm{y}$ coordinates and velocities of various eye movements, were recorded in “.avi” and “.xls” files respectively. Three repeat measurements were done with each subject on different days, aside for the case of one subject, who could only participate twice. 


\section{Section 9.4}

\section{Data Analysis}

The goal of the experiment was to determine if a central gaze fixating stimulus can be designed for Mapcatsf, which involves least variation of gaze position due to fixational eye movements. With the assumption that amplitudes of the fixational eye movements associated with foveal vision are the same in all directions, the fixation points were assumed to lie within a region which would be defined by a circle with the average of the recorded gaze coordinates as the center of the circle. The distance of the gaze positions with respect to the average of the fixation coordinates taken as the reference point was computed by using Eq. 1 as shown below.

$$
\left(x-x_{\text {average }}\right)^{2}+\left(y-y_{\text {average }}\right)^{2}=r^{2}
$$

The gaze positions as recorded by the software have normalized values between 0 and 1 for both the horizontal and vertical directions, as shown below in Fig 9.5. 


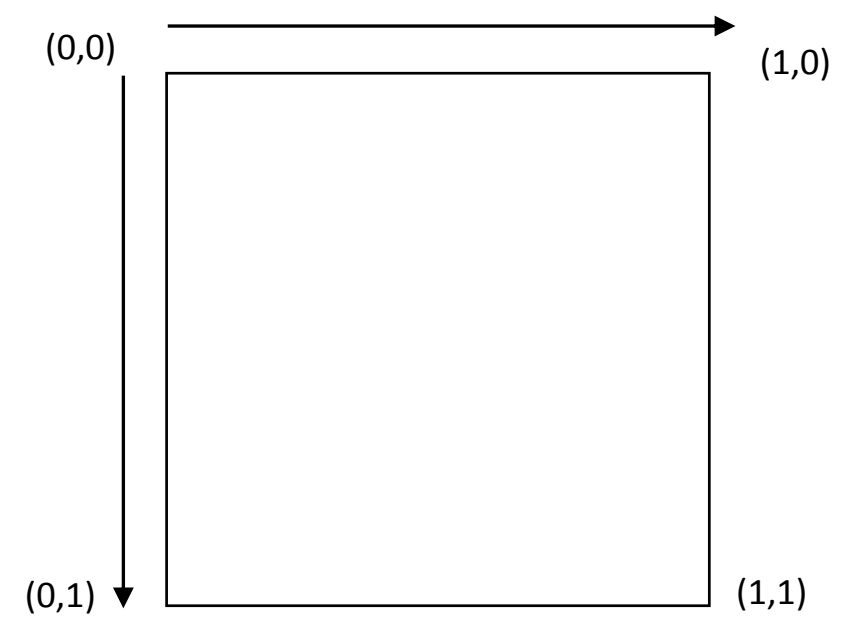

Fig. 9.5 Format of the scene as recorded

However on actual measurement, the extremities of the screen which are also the extremities of the field of view of the scene camera in the $\mathrm{x}$ and $\mathrm{y}$ directions were 49 inches and 40 inches respectively. Therefore the $\mathrm{x}$ and y coordinates recorded were multiplied by 49 and 40 respectively and were termed as converted x and y coordinates respectively. If any of the converted gaze coordinates were found far away from the average of the distribution, the entire frame associated with the coordinate was rejected. Such outliers in data are believed to be associated with blinks or saccades. 


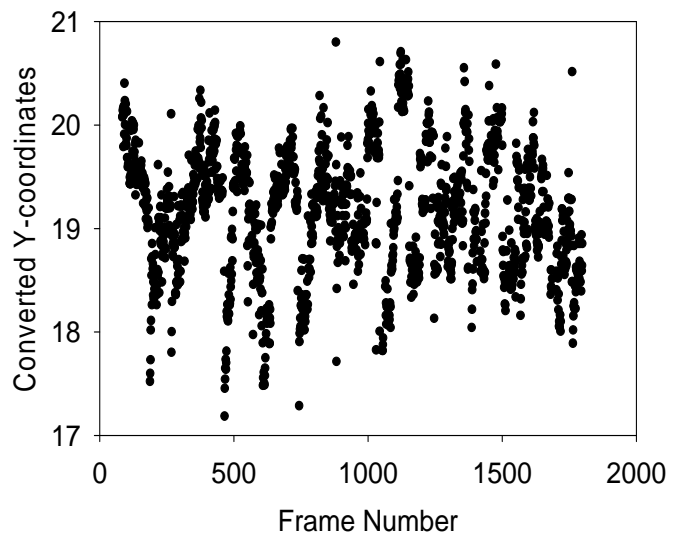

Fig 9.6 A sample of Transformed gaze coordinates in the $\mathrm{Y}$ direction prior to analysis.

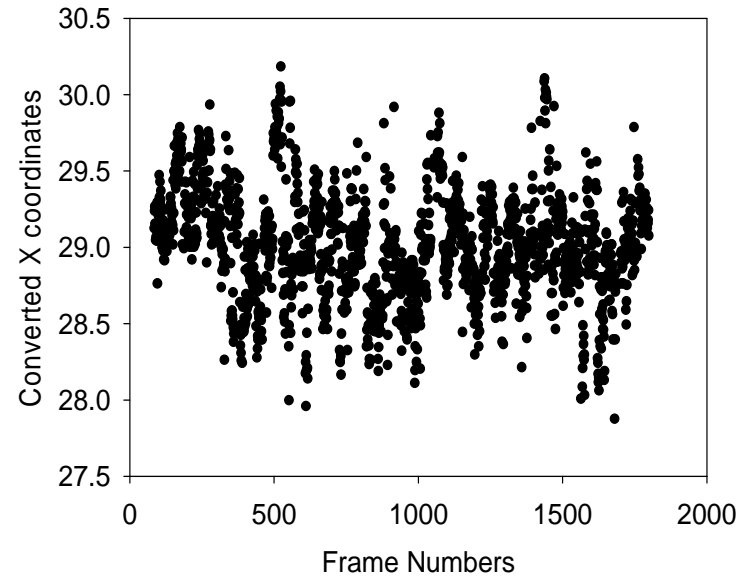

Fig 9.7 A sample of Transformed gaze coordinates in the $\mathrm{X}$ direction prior to analysis.

Since the eccentricities with respect to the visual axis are given in terms of angles, each fixation point was converted to angular eccentricities by the Eq.2:

$$
\theta=\tan ^{-1}\left(\frac{r}{73.5}\right)
$$


The number in the denominator of Eq. 2 is the distance in inches from the headmount to the screen.

The standard deviation in $\theta$ was interpreted as a measure of the variation in gaze position. A comparative analysis was done to statistically confirm the type of central gaze facilitating stimulus which resulted in the minimum variation of the gaze. A repeat measurement analysis of variance (ANOVA) was performed using the SPSS ${ }^{\mathrm{TM}}$ software. The results are summarized in table 9.1.

\begin{tabular}{|l|l|l|l|l|l|l|}
\hline $\begin{array}{l}\text { Expt. } \\
\text { No. }\end{array}$ & $\begin{array}{l}\text { No. of } \\
\text { Readings }\end{array}$ & $\begin{array}{l}\text { Type of } \\
\text { Stimulus }\end{array}$ & $\begin{array}{l}\text { Minimum } \\
\text { Variation } \\
\text { in gaze } \\
\left({ }^{\circ}\right)\end{array}$ & $\begin{array}{l}\text { Maximum } \\
\text { Variation } \\
\text { in gaze }\left(^{\circ}\right)\end{array}$ & $\begin{array}{l}\text { Mean } \\
\text { Variation } \\
\text { in gaze }\left({ }^{\circ}\right)\end{array}$ & $\begin{array}{l}\text { Standard } \\
\text { Deviation in } \\
\text { gaze }\left({ }^{\circ}\right)\end{array}$ \\
\hline 1 & 29 & $\begin{array}{l}\text { Vertical } \\
\text { Cross }\end{array}$ & 0.16 & 0.58 & 0.35 & 0.11 \\
\hline 2 & 29 & Dot & 0.14 & 0.72 & 0.35 & 0.14 \\
\hline 3 & 29 & X-cross & 0.17 & 0.67 & 0.37 & 0.13 \\
\hline
\end{tabular}

Table 9.1 Variation in gaze for the three types of stimuli tested.

The column "No. of readings" in table 9.1 implies the total of the three sittings for 9 subjects and the 2 sittings for the subject who could not participate for the third sitting. An F-test was further carried out to gain an understanding of the comparative variation in the gaze for the three types of stimuli tested among the subjects. The F-value ${ }^{144}$ is defined by Eq. (3).

$$
F=\frac{\text { between-group variability }}{\text { within group variability }}
$$


In Eq. 3 there are 2 degrees of freedom associated with the numerator and 3times (No. of readings-1) degrees of freedom associated with the denominator. From a look up table, the critical F-value was 3.11 for the p-value to be $<0.05$. The Fvalue for our data set was 0.617 calculated from the SPSS ${ }^{\mathrm{TM}}$ software and gave a p-value of 0.543 which is statistically insignificant.

\section{Section 9.5}

\section{Discussion and Conclusion}

Since The F-value we obtained using the SPSS ${ }^{\mathrm{TM}}$ software was less than the critical F-value, there is no significant difference between the level of influence that fixational eye movements might have on facilitating central gaze fixation for the three types of stimuli. From a statistical standpoint, selecting a particular type of central gaze fixating stimulus cannot significantly reduce fixational eye movements. Hence we surmise that though developers of HFP instruments might be tempted to consider an optimum gaze fixating stimulus, results of our experiment show that it might not be an effective way to reduce the effects of fixational eye movements on MPOD measurement. 


\section{Chapter 10}

\section{Conclusion}

Measuring and monitoring MP and the lens optical density for research and clinical purposes is the intended area where we would desire Mapcatsf, the novel LED-based HFP instrument to be used. The preliminary test-retest results of Mapcatsf for MPOD and LOD measurements as discussed in chapter 3 showed high levels of repeatability. This prompted us to further pursue the various testing and analyses as described in the later chapters of the dissertation.

In chapter 4 we observed that if changing either of the component LEDs is necessary, for maintenance of precise standards of measurement it is better to reprogram the microprocessor taking into account the spectrum of the replaced LEDs. Mapcatsf incorporates the age-related change in the luminous efficiency function. The outcome of chapter 5 is directed towards researchers who have reported an age-related decline in MPOD values measured by HFP. For the sake of improving the precision of their results, they are advised to consider the importance of incorporating the changing levels of the luminous efficiency function with age in their instruments which otherwise might lead to erroneous outcomes.

The true novel aspect of Mapcatsf being its capacity to measure LOD is further established when, in chapter 6, results are compared for the same group of subjects having their lens densities measured at absolute scotopic thresholds. A good level of agreement obtained between the results of the LOD interpreted in 
terms of LEA under the two separate viewing conditions further established the novel feature of Mapcatsf of being capable of measuring the LOD.

As supplementation studies to raise the levels of MP represent a growing trend among researchers, the capability of Mapcatsf to monitor changing levels of MPOD in individuals was tested and established as described in chapter 7. MPOD measured by HFP has been reported to represent the value at the edge of the foveal stimulus used. As described in chapter 9, this observation was attributed to fixational eye movements. Involvement of fixational eye movements with measured values of MPOD prompted us to test for an optimum gaze fixating stimulus which could be incorporated in Mapcasf. An eye tracking experiment pursued with 10 subjects led us to the inference that the current Mapcat stimulus is essentially optimized.

The overall outcome of the study establishes the credibility of Mapcatsf as a research grade instrument in labs and clinical settings. Further, if a future study is conducted where the same subject's MPOD is measured both by Mapcatsf and by one of the other such similar instrument available commercially, results from such a study would enable Mapcatsf to further stand out amongst its competitors. 


\section{REFERENCES}

1. Snodderly, D. M., Brown, P., Delori, F. \& Auran, J. The macular pigment. I. Absorbance spectra, localization, and discrimination from other yellow pigments in primate retinas. IOVS 25, 660-673 (1984).

2. Snodderly, D., Auran, J. \& Delori, F. The macular pigment II. Spatial distribution in primate retinas. IOVS 25, 674-85 (1984).

3. Jonas, J., Schneider, U. \& Naumann, G. Count and density of human retinal photoreceptors. Graefes Arch Clin Exp Ophthalmol 230, 505-510 (1992).

4. Bone, R. A. \& Landrum, J. Macular Pigment in Henle fiber membranes: a model for Haidinger brushes. Vision Res. 24, 103-108 (1984).

5. Rapp, L. M., Maple, S. S. \& Choi, J. H. Lutein and Zeaxanthin Concentrations in Rod Outer Segment membranes from Perifoveal and Peripheral Human Retina. IOVS 41, 1200-1209 (2000).

6. Malinow, M. R., Burns-Feeney, L., Peterson, L. H., Klein, M. \& Neuringer, M. Diet-related macular anomalies in monkeys. IOVS 19, 857-863 (1980).

7. Hermenger, R. Dichroism of the macular pigment and Haidinger's brushes. J.Opt.Soc.Am. 72, 734-737 (1982).

8. Maxwell, J. C. On the Theory of Compound Colours, and the relations of the Colours of the Spectrum. Philos. Trans. R. Soc. Lond. 150, 57-84 (1860).

9. Bone, R. A., Landrum, J. T. \& Cains, A. Optical density spectra of the macular pigment in vivo and in vitro. Vision Res. 32, 105-110 (1992). 
10. Shute, C. C. D. in The McCollough Effect: An Indicator of Central Neurotransmitter Activity 33 (Cambridge University Press 1979, 1979).

11. Bone, R. A., Landrum, J. \& Tarsis, S. Preliminary Identification of the Human Macular Pigment. Vision Res. 25, 1531-1535 (1985).

12. Handelman, G., Dratz, E., Reay, C. \& van Kuijk, F. Carotenoids in the human macula and whole retina. IOVS 29, 850-855 (1988).

13. Bone, R., Landrum, J., Fernandez, L. \& Tarsis, S. Analysis of the Macular Pigment by HPLC:retinal distribution and Age Study. IOVS 29, 843-849 (1988).

14. Wald, G. Human vision and the spectrum. Science 101, 653-658 (1945).

15. Bone, R. \& Sparrock, J. Comparison of macular pigment densities in human eyes. Vision Res. 11, 1057-1064 (1971).

16. Landrum, J. T. \& Nolan, J. M. in Carotenoids and Retinal Disease 9 (CRC Press Taylor \&Francis Group., 2014).

17. Bone, R., Landrum, J., Guerra, L. \& Ruiz, C. Lutein and zeaxanthin dietary supplements raise macular pigment density and serum concentrations of these carotenoids in humans. J. Nutr. 133, 992-998 (2003).

18. Harrison, E. R. Visual Acuity and the Cone Cell Distribution of the Retina. Br. J. Ophthalmol. 37, 538-542 (1953).

19. Bressler, N. M., Bressler, S. B. \& Fine, S. L. Age-related macular degeneration. Surv. Ophthalmol. 32, 375-413 (1988). 
20. Jager, R. D., William F. Mieler M.D. \& Miller, J. W. Age-Related Macular Degeneration. N. Engl. J. Med. 358, 2606-2617 (2008).

21. Haines, J. L., Hauser, M. A., Schmidt, S. \& Scott, W. K. Complement Factor $\mathrm{H}$ Variant Increases the Risk of Age-Related Macular Degeneration. Science 308, (2005).

22. Suzuki, M. et al. Chronic photo-oxidative stress and subsequent MCP-1 activation as causative factors for age-related macular degeneration. $J$ Cell Sci 125, 2407-15 (2012).

23. Yu, D. \& Cringle, S. Oxygen distribution and consumption within the retina in vascularized and avascular retinas and in animal models of retinal disease. Prog Retin Eye Res 20, 175-208 (2001).

24. Margrain, T., Boulton, M., Marshall, J. \& Sliney, D. Do blue light filters confer protection against age-related macular degeneration? Prog Retin Eye Res 23, 523-531 (2004).

25. Haegerstrom-Portnoy, G. Short-wavelength-sensitive-cone sensitivity loss with aging: a protective role for macular pigment? JOptSocAm A 5, 2140 2144 (1988).

26. Werner, J. S., Bieber, M. L. \& Schefrin, B. E. Senescence of foveal and parafoveal cone sensitivities and their relations to macular pigment density. JOptSocAm A 17, 1918-1932 (2000).

27. Bernstein, P. S., Delori, F. C., Richer, S., van Kujik, F. J. M. \& Wenzel, A. J. The Value of Measurement of Macular Carotenoid Pigment Optical Densities and Distributions in Age-Related Macular Degeneration and Other Retinal Disorders. Vision Res. 50, 716-728 (2010). 
28. Snodderly, D. Evidence for protection against age-related macular degeneration by carotenoids and antioxidant vitamins. Am J Clin Nutr 62, 1448S-1461S (1995).

29. Vrensen, G. Early cortical lens opacities: a short overview. Acta Ophthalmol. (Copenh.) 87, 602-610 (2009).

30. Collier, R. J. et al. Agonists at the Serotonin Receptor (5-HT1A) Protect the Retina from Severe Photo-Oxidative Stress. IOVS 52, 2118-2126 (2011).

31. Ciulla, T. A., Hammond, Jr., B. R., Yung, C. W. \& Pratt, L. M. Macular Pigment Optical Density before and after Cataract Extraction. IOVS 42, 1338-1341

32. Ermakov, I. V., Ermakova, M. R. \& Gellermann, W. Simple Raman Instrument for in Vivo Detection of macular Pigments. Appl. Spectrosc. 59, 861-867 (2005).

33. Delori, F., Goger, D., Hammond, B., Snodderl, D. \& Burns, S. Macular pigment density measured by autofluorescence spectrometry: Comparison with reflectometry and heterochromatic flicker photometry. J Opt Soc Am Opt Image Sci Vis 18, 1212-30 (2001).

34. Berendschot, T. T. J. M. \& van Norren, D. Objective determination of the macular pigment optical density using fundus reflectance spectroscopy. Arch. Biochem. Biophys. 430, 149-155 (2004).

35. Bone, R. A. \& Landrum, J. T. Heterochromatic flicker photometry. Arch. Biochem. Biophys. 430, 137-142 (2004). 
36. Snodderly, D. et al. Macular pigment measurement by heterochromatic flicker photometry in older subjects:the carotenoids and age related eye disease study. IOVS 45, 531-538 (2004).

37. Kraft, J. \& Werner, J. S. Spectral Efficiency across the life span: flicker photometry and brightness matching. JOptSocAm A 11, 1213-1221 (1994).

38. Siik, S. LENS AUTOFLUORESCENCE In aging and cataractous human lenses. Clinical applicability. (Department of Ophthalmology, University of Oulu, 1999). at <http://herkules.oulu.fi/isbn9514252675/isbn9514252675.pdf>

39. Klein, R., Klein, E. K. \& Linton, K. Prevalence of age-related maculopathy:The Beaver Dam Eye Study. Ophthalmology 99, 933-943 (1992).

40. Werner, J. S. \& Wooten, B. R. Opponent chromatic response functions for an average observer. Percept Psychophys 25, 371-374 (1979).

41. Wooten, B., Hammond, B., Land, R. \& Snodderly, D. A practical method for measuring macular pigment optical density. IOVS 40, 2481-2489 (1999).

42. Mellerio, J. et al. A portable instrument for measuring macular pigment with central fixation. Curr. Eye Res. 25, 37-47 (2002).

43. Bone, R. A. \& Mukherjee, A. Innovative Troxler-free measurement of macular pigment and lens density with correction of the former for the aging lens. J. Biomed. Opt. 18, (2013).

44. Kinkelder, R. de, van der Veen, R., Verbaak, F. \& Faber DJ. Macular pigment optical density measurements: evaluation of a device using heterochromatic flicker photometry. Eye 25, 105-112 (Nov 5). 
45. Hagen, S., Krebs, I., Glittenberg, C. \& Binder, S. Repeated measures of macular pigment optical density to test reproducibility of heterochromatic flicker photometry. Acta Ophthalmol. (Copenh.) 88, 207-211 (2010).

46. Pokorny, J., Smith, V. C. \& Lutze, M. Aging of the human lens. Appl. Opt. 26, $1437-1440$

47. Van de Kraats, J. \& van Norren, D. Optical density of the aging human ocular media in the visible and UV. JOSA 24, 1842-1857 (2007).

48. Bone, R. A., Landrum, J. T. \& Gibert, J. C. Macular pigment and the edge hypothesis of flicker photometry. Vision Res. 44, 3045-3051 (2004).

49. Martinez-Conde, S. Fixational eye movements in normal and pathological vision. Prog. Brain Res. 154, 151-176 (2006).

50. Chung, S. T. L., Kumar, G., Li, R. W. \& Levi, D. M. Characteristics of fixational eye movements in amblyopia:Limitations on fixation stability and acuity? Vision Res. ahead of print,

51. Bone, R. \& Landrum, J. Distribution of macular pigment components,zeaxanthin and lutein, in human retina. Methods Enzym. 213, 360-366

52. Sagawa, K. \& Takahashi, Y. Spectral luminous efficiency as a function of age. JOSA 18, 2659-2667 (2011).

53. Stringham, J. et al. The utility of using customized heterochromatic flicker photometry (cHFP) to measure macular pigment in patients with age related macular degenration. Exp Eye Res 87, 445-453 (2008). 
54. Van der Veen, R., Berendschot, T., Carden, D., Maknidaki, M. \& Murray, I. A new desktop instrument for measuring macular pigment optical density based on a novel technique for setting flicker thresholds. Ophthalmic Physiol Opt 29, 127-137 (2009).

55. Kanis, M., Berendschot, T. \& van Norren, D. Interocular agreement in melanin and macular pigment optical density. Exp Eye Res 84, 934-938 (2007).

56. Wooten, B. R., Hammond, B. R. \& Renzi, L. M. Using scotopic and photopic flicker to measure lens optical density. Ophthalmic Physiol. Opt. 27, 321-328 (2007).

57. Loane, E., Stack, J., Beatty, S. \& Nolan, J. M. Measurement of Macular Pigment Optical Density Using Two Different Heterochromatic Flicker Photometers. Curr. Eye Res. 32, 555-564 (2007).

58. Nolan, J. et al. Macular pigment and percentage of body fat. IOVS 45, 39403950 (2004).

59. Yu, J. et al. Measurement of macular pigment optical density in a healthy Chinese population sample. IOVS 53, 2106-2111 (2012).

60. Hofer, H., Carroll, J., Nietz, J., Neitz, M. \& Williams, D. R. Organization of the human trichromatic cone mosaic. J Neurosci. 25, 9669-9679 (2005).

61. Carroll, J., McMahon, C. \& Nietz, J. Flicker-photometric electroretinogram estimates of L:M cone photoreceptor ratio in men with photopigment spectra derived from genetics. JOptSocAm A 17, 499-509 (2000).

62. Van Norren, D. V. \& Vos, J. J. Spectral Transmission of the human ocular media. Vision Res. 14, 1237-1244 (1974). 
63. Hammond, B. R. et al. Dietary Modification of Human Macular Pigment Density. IOVS 38, 1795-1801 (1997).

64. Hammond, B., Wooten, B. \& Snodderly, D. Density of the human crystalline lens is related to the macular pigment carotenoids, lutein and zeaxanthin. Optom Vis Sci 74, 499-504 (1997).

65. Ham, W. J. Ocular hazards of light sources:review of current knowledge. $J$. Occup. Med. 25, 101-103 (1983).

66. Beatty, S., Koh, H.-H., Carden, D. \& Murray, I. J. Macular Pigment optical density measurement: A novel compact instrument. Ophthalmic Physiol. Opt. 20, 105-111

67. Hammond, B. \& Caruso-Avery, M. Macular pigment optical density in a southwestern sample. IOVS 41, 1492-1497 (2000).

68. Dietzel, M., Zeimer, M., Claes, B., Pauleikhoff, D. \& Hense, H.-W. Determinants of Macular Pigment Optical Density and Its Relation to AgeRelated Maculopathy: Results from the Muenster Aging and Retina Study (MARS). IOVS 52, 3452-3457 (2011).

69. Liew, S. et al. Heritability of macular pigment: a twin study. IOVS 38, 17951801 (1997).

70. Berendschot, T., Willemse-Assinck, J., Bastiaanse, M., Jong PTVM, de \& van Norren, D. Macular pigment and melanin in age-related maculopathy in a general population. IOVS 43, 1928-1932 (2002).

71. Bernstein, P., Zhao, D., Sharifzadeh, M., Ernakov, I. \& Gellermann, W. Resonance Raman measurement of macular carotenoids in the living human eye. Arch Biochem Biophys 430, 163-169 (2004). 
72. Ciulla, T., Curran-Celentano, J., Cooper, D. \& Hammond, B. Macular pigment optical density in a midwestern sample. Ophthalmology 108, 730737 (2001).

73. Neelam, K., O’Gorman, N., Nolan, J., Wong, H. \& Eong, K. Measurement of macular pigment: Raman spectroscopy versus versus heterochromatic flicker photometry. IOVS 46, 1023-1032 (2005).

74. Mellerio, J., Ahmadi, L., van Kujik, F. \& Pauleikhoff, D. A portable instrument for measuring macular pigment with central fixation.

75. Stiles, W. \& Burch, J. NPL colour-matching investigation:final report. Opt.Acta 6, 1-26 (1959).

76. Michael, R. \& Bron, A. J. The ageing lens and cataract: a model of normal and pathological ageing. Philos. Trans. R. Soc. Biol. Sci. 366, 1278-1292 (2011).

77. Roodhooft, J. Leading causes of blindness worldwide. Bull Soc Belge Ophtalmol 283, 19-25 (2002).

78. Taylor, V. L. et al. Morphology of the Normal Human Lens. IOVS 37, 1396-1410 (1996).

79. Beebe, D. C., Holekamp, N. M. \& Shui, Y.-B. Oxidative Damage and the Prevention of Age-Related Cataracts. Ophthalmic Res. 44, 155-65 (2010).

80. Age-Related Eye Disease Study Research Group. Risk Factors Associated with Age-Related Nuclear and Cortical Cataract:A Case-control Study in the Age-Related Eye Disease Study, AREDS Report No.5. Ophthalmology 108, 1400-1408 (2001). 
81. Shiels, PhD, A. \& Hejtmancik, F. J. Genetic Origins of Cataract. Arch Ophthalmol 125, 165-173 (2007).

82. Cumming, R. \& Mitchell, P. Alcohol, smoking, and cataracts: the Blue Mountains Eye Study. Arch Ophthalmol 115, 1296-1303 (1997).

83. Roberts, J. Ultraviolet radiation as a risk factor for cataract and macular degeneration. Eye Contact Lens 37, 246-249 (2011).

84. Savage, G., Johnson, C. \& Howard, D. A comparison of noninvasive objective and subjective measurements of the optical density of human ocular media. Optom Vis Sci 78, 386-395 (2001).

85. Bleeker, J., van Best, J., Vrij, L., van der Velde, E. A. \& Oosterhuis, J. . Autofluorescence of the lens in diabetic and healthy subjects by fluorophotometry. IOVS 27, 791-794 (1986).

86. Delori, F. C. \& Burns, S. Fundus Reflectance and the Measurement Of Crystalline Lens Density. JOSA 13, 215-226 (1996).

87. Teikari, P. et al. Refined Flicker Photometry technique to measure Ocular lens Density. JOSA 29, 2469-2478 (2012).

88. Davies, N. \& Morland, A. Color Matching in Diabetes: Optical Density of Crystalline Lens and Macular Pigments. IOVS 43, 281-289 (2002).

89. Sharpe, L. T., Stockman, A., Jagla, W. \& Jagle, H. A luminous efficiency function, $\mathrm{V}^{*}$, for daylight adaptation. J. Vis. 5, 948-968 (2005).

90. Wright, W. The visual sensitivity of normal and aphakic observers in the ultraviolet. Ann. Psychol. 50, 169-177 (1951). 
91. Hood, D. \& Finkelstein, M. A. in Handbook of Perception and Human Performance (Wiley, 1986).

92. Glaser, J. S. in Neuro-Ophthalmology 8 (Lippincott Williams \& Wilkins, a Wolter Kluwer business, 1999).

93. Crescetelli, F. \& Dartnall, H. Human visual purple. Nature 172, 195-197 (1953).

94. Landrum, J. \& Bone, R. A. Lutein, zeaxanthin, and the macular pigment. Arch. Biochem. Biophys. 385, 28-40 (2001).

95. Nolan, J. M., Meagher, K., Kashani, S. \& Beatty, S. What is mesozeaxanthin, and where does it come from? Eye 27, 899-905 (2013).

96. Nolan, J. et al. Augmentation of macular pigment following implantation of blue light-filtering intraocular lenses at the time of cataract surgery. IOVS 50, 4777-85 (2009).

97. Bone, R. A., Brener, B. \& Gibert, J. C. Macular pigment,photopigments and melanin:Distributions in young subjects determined by four-wavelength reflectometry. Vision Res. 47, 3259-3268 (2007).

98. Ciulla, T. A., Harris, A. \& Martin, B. J. Ocular perfusion and age-related macular degeneration. Acta Ophthalmol. Scand. 79, 108-115 (2002).

99. Beatty, S., Frederik, J. G. M. van K. \& Chakravarthy, U. Macular Pigment and Age-Related Macular Degeneration: Longitudinal Data and Better techniques of Measurement are Needed. IOVS 49, 843-845 
100. Qingning, B. et al. Lutein and zeaxanthin supplementation reduces photooxidative damage and modulates the expression of inflammation-related genes in retinal epithelial cells. Free Radic Biol Med 53, 1298-1307 (2012).

101. Hammond, B. R., Johnson, E. J. \& Russe. Dietary Modification of Human Macular Pigment Density.

102. Howells, O., Eperjesi, F. \& Bartlett, H. Measuring macular pigment optical density in vivo: a review of techniques. Graefes Arch Clin Exp Ophthalmol 249, 315-347 (2011).

103. Ermakov, I. V., Ermakova, M. R. \& Gellermann, W. Simple Raman Instrument for in Vivo Detection of macular Pigments. Appl. Spectrosc. 59, 861-867 (2005).

104. Bone, R. \& Sparrock, J. Comparison of macular pigment densities in human eyes. Vision Res. 11, 1057-1064 (1971).

105. Beatty, S. et al. Macular pigment and risk for age-related macular degeneration in subjects from a Northern European population. IOVS 42, 439-446 (2001).

106. Beatty, S., Koh, H.-H., Carden, D. \& Murray, I. J. Macular Pigment optical density measurement: A novel compact instrument. Ophthalmic Physiol. Opt. 20, 105-111

107. Nolan, J. et al. Macular pigment and percentage of body fat. IOVS 45, 39403950 (2004).

108. Roodhooft, J. Leading causes of blindness worldwide. Bull Soc Belge Ophtalmol 283, 19-25 (2002). 
109. Sommerburg, O., Keunen, J. E. E., Bird, A. C. \& Kujik, F. J. G. M. Fruits and vegetables that are sources for lutein. Br. J. Ophthalmol. 82, 907-910 (1998).

110. Bone, R., Landrum, J., Guerra, L. \& Ruiz, C. Lutein and zeaxanthin dietary supplements raise macular pigment density and serum concentrations of these carotenoids in humans. J. Nutr. 133, 992-998 (2003).

111. Bone, R. A., Landrum, J. T., Cao, Y., Howard, A. N. \& Alvarez-Calderon, F. Macular pigment response to a supplement containing meso-zeaxanthin, lutein and zeaxanthin. Nutr. Metab. 4, (2007).

112. Landrum, J. et al. A one year study of the macular pigment: the effect of 140 days of a lutein supplement. Exp Eye Res 65, 57-62 (1997).

113. Murray, I. et al. Lutein supplementation over a one-year period in early AMD might have a mild beneficial effect on visual acuity: the CLEAR study. IOVS 54, 1781-1788 (2013).

114. Bone, R. A. \& Landrum, J. T. Dose-dependent response of serum lutein and macular pigment optical density to supplementation with lutein esters. Arch Biochem Biophys 504, 50-55 (2010).

115. Weigert, G. et al. Effects of lutein supplementation on macular pigment optical density and visual acuity in patients with age-related macular degeneration. IOVS 52, 8174-8178 (2011).

116. Pigmenting eggs and broiler chickens. at $<$ http://www.dsm.com/markets/anh/en_US/products/productscarotenoids/world_egg_day/Pigmenting_eggs_and_broiler_chickens.html $>$

117. Goralzyck, R. Beta-carotene and lung cancer in smokers: review of hypotheses and status of research. Nutr Cancer 61, 767-774 (2009). 
118. Horwitz, J. Alpha-crystallin. Exp. Eye Res. 76, 145-153 (2003).

119. Iribarren, R., Morgan, I. G., Nangia, V. \& Jonas, J. B. Crystalline Lens Power and Refractive Error. IOVS 53, 543-550 (2012).

120. Weale, R. A. Age and the transmittance of the human crystalline lens. $J$ Physiol 395, 577-587 (1988).

121. Mellerio, J. Yellowing Of the Human Lens: Nuclear and Cortical Contributions. Vision Res. 27, 1581-1587 (1987).

122. Delcourt, C. et al. Risk Factors for Cortical, Nuclear, and Posterior Subcapsular Cataracts the POLA Study. Am. J. Epidemiol. 151, 497-504 (2000).

123. Hiller, R., Sperduto, R. \& Ederer, F. Epidemiologic associations with nuclear, cortical and posterior subcapsular cataracts. Am. J. Epidemiol. 124, 916-925 (1986).

124. Chodick, G., Bekiroglu, N., Hauptmann, M., Doody, M. M. \& Cheung, L. C. Risk of Cataract after Exposure to Low Doses of Ionizing Radiation: A 20Year Prospective Cohort Study among US Radiologic Technologists. Am. J. Epidemiol. 168, 620-631

125. Spector, A. Oxidative stress-induced cataract:mechanism of action. FASEB $J$ 9, 1173-1182 (1995).

126. Varma, S., Kovtun, S. \& Hegde, K. Role of ultraviolet irradiation and oxidative stress in cataract formation-medical prevention by antioxidants and metabolic agonists. Eye Contact Lens 37, 233-45 (2011). 
127. Beebe, D. C., Holekamp, N. M., Siegfried, C. \& Shui, Y.-B. Vitroretinal influences on lens function and cataract. Philos. Trans. R. Soc. Biol. Sci.

128. Dairou, J., Malecaze, F., Dupret, J. M. \& Lima, F. R. The XenobioticMetabolizing Enzymes Arylamine N-Acetyltransferases in Human Lens Epithelial Cells: Inactivation by Cellular Oxidants and UVB-Induced Oxidative Stress. Mol. Pharmacol. 67, 1299-1306 (2005).

129. Gao, S., Qin, T., Liu, Z., Caceras, M. A. \& Ronchi Carlos F. Lutein and zeaxanthin supplementation reduces H2O2-induced oxidative damage in human lens epithelial cells. Mol. Vis. 17, 3180-3190 (2011).

130. Davies, K. Protein oxidation and proteolytic degradation. General aspects and relationship to cataract formation. Adv Exp Med Biol 264, 503-511

131. Curran-Celentano, J. et al. Relation between dietary intake, serum concentrations, and retinal concentrations of lutein and zeaxanthin in adults in a Midwest population. Am J Clin Nutr 74, 796-802 (2001).

132. Berendschot, T. T. J. M., Broekmans, W., Ineke, A. \& Klopping-Ketelaars, A. Lens Aging in Relation to Nutritional Determinants and Possible Risk Factors for Age-Related Cataract. JAMA Ophthalmol. 120, 1732-1737 (2002).

133. Chasan-Taber, L., Willett, W., Seddon, J. \& Stampfer, M. A prospective study of carotenoid and vitamin A intakes and risk of cataract extraction in US women. Am J Clin Nutr 70, 509-516 (1999).

134. Kanis, M., Berendschot, T. \& van Norren, D. Interocular agreement in melanin and macular pigment optical density. Exp. Eye Res. 934-8 (2007). 
135. Sommerburg, O., Keunen, J. E. E., Bird, A. C. \& Kujik, F. J. G. M. Fruits and vegetables that are sources for lutein. Br. J. Ophthalmol. 82, 907-910 (1998).

136. Kopsell, D. E., Kopsell, D. A., Randle, W. M. \& Coolong, T. W. Kale Carotenoids Remain Stable while Flavor Compounds Respond to Changes in Sulfur Fertility. J. Agric. Food Chem. 51, (2003).

137. Klein, B., Klein, R. \& Lee, K. Incidence of age-related cataract: the Beaver Dam. Arch Ophthalmol 116, 219-225 (1998).

138. Bour, L. J., Koo, L., Delori, F. C., Apkarian, P. \& Fulton, A. B. Fundus photography for measurement of macular pigment density distribution in children. IOVS 43, 1450-1455 (2002).

139. Chen, S.-J., Chang, Y. C. \& Wu, J. C. The spatial distribution of macular pigment in humans. Curr. Eye Res. 23, 422-434 (2001).

140. Elsner, A. E., Burns, S. A., Beausencourt, E. \& Weiter, J. J. Foveal cone photopigment distribution:Small alterations associated with macular pigment distribution. IOVS 39, 2394-2404 (1998).

141. Hammond, B. J., Wooten BR \& Snodderly, D. Individual variations in the spatial profile of human macular pigment. J Opt Soc Am Opt Image Sci Vis 14, 1187-1196

142. Clowes, M. B. A note on colour discrimination under conditions of retinal image constraint. Opt.Acta 8, 121-135

143. Modified URL:

http://www.arringtonresearch.com/ViewPointPCWindow.html. 
144. URL: http://www.bodowinter.com/tutorial/bw_anova_general.pdf.

145. URL: http://www.danielsoper.com/statcalc3/calc.aspx?id=4. 


\section{VITA}

ANIRBAAN MUKHERJEE

2009-2015

$\mathrm{PhD}$ in Physics

Florida International University

Miami Florida, USA

2007-2009

M.S in Physics

California State University Fresno

Fresno, California

1999-2002

B.Sc Physics(Hons.)

University of Calcutta

Calcutta, India

WORK EXPERIENCE

2003-2007

Marketing and Liaison Officer

Arunodoy Enterprise

Kolkata, India

TEACHING EXPERIENCE

2007-2009

Graduate Teaching Assistant

Department of Physics

California State University, Fresno

Fresno, California, USA

2009-2015
Graduate Teaching Assistant
Department of Physics
Florida International University
Miami, Florida,USA

\section{PUBLICATIONS AND PRESENTATIONS}

1) "Innovative Troxler-free measurement of macular pigment and lens density with correction of the former for the aging lens.” Richard A Bone and Anirbaan Mukherjee. J. Biomed.Opt.18(10),107003 ( Oct. 10, 2013 )

2) "Light distributions on the retina: relevance to macular pigment photoprotection.” Richard A Bone, Jorge C. Gibert, Anirbaan Mukherjee, Acta Biochim. Polon. 59,167-169 (2012 ) 
3) Chapter 12 "Light Distribution on the Retina: Implications for AgeRelated Macular Degeneration.” Richard A. Bone, Jorge C. Gibert and Anirbaan Mukherjee. Carotenoids and Retinal Disease , Edited By John T. Landrum and John M. Nolan. CRC Press, Taylor \& Francis Group, New York, NY, USA.

4) ARVO 2015 (Denver, Colorado, USA):(Poster) "Validation of the Mapcat for Lens Density Measurements”. Anirbaan Mukherjee, Richard A. Bone and Miguel A. Escanelle.

5) ARVO 2014 (Orlando, Florida, USA): (Poster)“Three Characteristics of the Central Macula : Light, AMD and Macular Carotenoids”. Richard A. Bone, Jorge C. Gibert, Anirbaan Mukherjee

6) Macular Carotenoids Conference 2013 (Cambridge, UK): (Poster +Oral) "Repeatability of a New Heterochromatic Flicker Photometer for Measuring Macular Pigment”Anirbaan Mukherjee, Richard A. Bone.

7) ARVO 2013(Seattle, Washington, USA): (Poster) "Evaluation of a novel instrument for simultaneous measurement of macular pigment and lens optical density, and lens equivalent age”. Richard A Bone, Anirbaan Mukherjee, Jeffrey Morris.

8) ARVO 2012(Fort Lauderdale, Florida, USA): (Poster) “Age-Related Decline in Macular Pigment with LED-based Flicker: a Possible Artifact of the Aging Lens.” Anirbaan Mukherjee, Richard A. Bone, Jorge C. Gibert.

9) ARVO 2012 (Fort Laudedale, Florida, USA): (Poster) "Innovative Troxler-free Measurement of Macular Pigment and Lens Density with Correction of the Former for the Aging Lens.” Richard A. Bone, Jeffrey B. Morris, Anirbaan Mukherjee.

10) ARVO 2011 (Fort Lauderdale, Florida, USA): (Poster) "Cumulative Light Distribution on the Human Retina: Implications for AMD." Jorge C Gibert, Richard A Bone, Anirbaan Mukherjee. 